\title{
Abstracts from the 2018 European Meeting on Psychosocial Aspects of Genetics
}

(c) European Society of Human Genetics 2019

June 16-19, 2018, Fiera Milano Congressi, Milan Italy

Sponsorship: Publication of this supplement was sponsored by the European Society of Human Genetics. All content was reviewed and approved by the ESHG Scientific Programme Committee, which held full responsibility for the abstract selections.

Disclosure Information: In order to help readers form their own judgments of potential bias in published abstracts, authors are asked to declare any competing financial interests.

Contributions of up to EUR 10 000.- (Ten thousand Euros, or equivalent value in kind) per year per company are considered "Modest". Contributions above EUR 10 000.- per year are considered "Significant".

\section{Oral presentations}

EBPL1 Hopes and expectations on genome editing

\section{EBPL1.1}

The ethics of clinical applications of germline genome modification:a systematic review of reasons

I. van Dijke $e^{1,2}$, L. Bosch ${ }^{2}$, A. L. Bredenord ${ }^{3}$, M. Cornel ${ }^{1}$, S. Repping ${ }^{2}$, S. Hendriks ${ }^{2,4}$

${ }^{1}$ VU University Medical Center, Amsterdam, Netherlands,

${ }^{2}$ Academic Medical Center, University of Amsterdam, Amsterdam, Netherlands, ${ }^{3}$ University Medical Center Utrecht, Utrecht, Netherlands, ${ }^{4}$ National Institutes of Health, Washington DC, DC, United States

Germline genome modification is still unsafe and insufficiently effective for clinical purposes. However, the progress made using CRISPR-Cas has led scientists to expect to overcome the technical hurdles in the foreseeable future. This has invited debate on the socio-ethical and legal implications. A systematic overview of reasons for being in favour or against germline genome modification is missing.

We systematically searched 'Medline/Pubmed' (January 2011 - June 2016) and included all types of articles except for original biological research, that covered reasons for or against clinical application of intentional modification of the nuclear DNA of the germline.
We identified 1179 articles of which 180 articles could be included. We identified 169 reasons, 90 reasons for and 79 reasons against future clinical application of germline genome modification. None of the included articles mentioned more than 60/169 reasons. The reasons could be categorised into: (i) quality of life of affected individuals; (ii) safety; (iii) effectiveness; (iv) existence of a clinical need or alternative; (v) costs; (vi) effects on homo sapiens as a species; (vii) social justice; (viii) potential for misuse; (ix) special interests exercising influence; (x) parental rights and duties; (xi) comparability to acceptable processes; (xii) rights of the unborn child; (xiii) human life and dignity.

Besides the need for biotechnical and clinical studies on safety and effectiveness, there is an evident need for a sound pre-implementation process. The provided overview of all reasons will aid in allowing for a systematic and thorough debate on the introduction of germline genome editing.

I. van Dijke: None. L. Bosch: None. A.L. Bredenord: None. M. Cornel: None. S. Repping: None. S. Hendriks: None.

\section{EBPL1.2}

Enabling informed opinions about germline editing among the general public: a pilot study

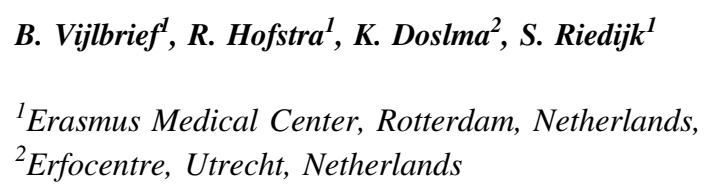


Background: The commonness of crispr-cas in the genetic community is in great contrast with the ignorance about it among the general public. A lack of initiative from the genetic community to bridge this gap may explain this. We aimed to inform the general Dutch public about germline editing and its potential use in the clinic in order to enable informed opinions.

Methods: Interested lay attendees of an informative afternoon containing three lectures about germline editing were invited to participate in a survey regarding their attitude and opinion towards germline editing before and after the lectures. A total of 39/66 attendees were included in both the pre and post afternoon survey (age $\bar{x}=57$ years; $54 \%$ completely lay).

Results: A shift in attitude towards germline editing in both directions was observed at post-measurement $(\mathrm{P}<.01)$. A significant shift $(\mathrm{P}<.01)$ was also found in the preferred source of information; generally from political entities to professional genetic organizations. Those favoring germline editing used significantly more sources of information than those opposing $(\mathrm{P}<.001)$. At pre-measurement, opposing attendees relied on opinions significantly more than those favoring germline editing $(\mathrm{P}=.05)$, whereas at the postmeasurement this ratio flipped.

Discussion/conclusion: Our pilot study demonstrates a significant impact of three lectures about germline editing on the attitudes of lay people. After having received information, lay people wished the genetic community to take responsibility for informing them about germline editing. This pilot study indicates a leading role for the genetic community in educating the public about germline editing.

B. Vijlbrief: None. R. Hofstra: None. K. Doslma: None. S. Riedijk: None.

\section{EBPL1.3}

Informed consent in a human germline gene editing study ethical issues

\section{E. Niemiec, H. C. Howard}

Uppsala University, Uppsala, Sweden

Recent discussions over ethical aspects of germline gene editing (GLGE) tend to focus on its potential clinical applications. This may somewhat eclipse the importance of addressing ethical aspects (e.g. adequacy of research participants' informed consent) of research involving GLGE. Genomic sequencing, which is usually part of such studies introduces an additional layer of ethical challenges. Ma et al. (2017) conducted one of the first studies in which CRISPR-Cas9 was used to correct a gene in viable embryos created for the purpose of this research. To provide insights into information provided to research participants (gamete donors) in this study, we analysed relevant informed consent forms focusing on information about sensitive nature of the study, genomic sequencing, and readability of the forms. Preliminary results show that some content pertaining to ethically sensitive nature of the study may be misleading, which raises doubts whether research participants could have given genuinely informed consent to the study. Although information about genetic testing was present, whole genome/exome sequencing was not explicitly mentioned. Questions arise whether lack of explicit consent for whole genome/exome sequencing is an adequate practice. Average reading grade level of informed consent forms was 13 (SMOG test), which is above the recommended grade level of 8. To respect research participants and maintain transparency and trust in research, informed consent documents should present accurate information. Debatable issues pertaining to adequacy of content of such documents for GLGE studies should be discussed and clarified among stakeholders, especially given that these studies are of ethically sensitive nature.

E. Niemiec: None. H.C. Howard: None.

\section{EBPL1.4}

The PRISM-IMPACT study: What are the hopes and expectations of families and health care professionals enrolling in a personalised medicine trial for high risk childhood cancers?

J. Vetsch ${ }^{1,2}$, C. E. Wakefield ${ }^{1,2}$, K. Tucker ${ }^{3}$, M. Warby ${ }^{3}$,

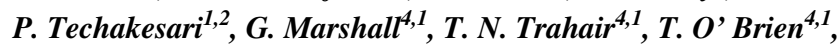
J. M. Marron ${ }^{5}$, L. Lau ${ }^{4,1}$, V. Tyrell ${ }^{6}$, D. Ziegler ${ }^{4,1}$

${ }^{1}$ School of Women's and Children's Health, UNSW Sydney, Sydney, NSW, Australia, ${ }^{2}$ Behavioural Sciences Unit, Kids Cancer Centre, Sydney Children's Hospital, Randwick, NSW, Australia, ${ }^{3}$ Hereditary Cancer Centre, Prince of Wales Hospital, Sydney, Australia, ${ }^{4}$ Kids Cancer Centre, Sydney Children's Hospital, Randwick, NSW, Australia, ${ }^{5}$ Department of Pediatric Oncology, Dana-Farber/Boston Children's Cancer and Blood Disorders Center, 450 Brookline Ave,, Boston, MA, United States, ${ }^{6}$ Children's Cancer Institute, UNSW Sydney, Sydney, Australia

Background: The PRISM precision medicine trial uses novel technologies (e.g. genomic, in vitro/in vivo drug testing) to identify personalised therapies for patients with high risk childhood cancers. PRISM-Impact aims to prospectively assess families' and health care professionals' (HCP) hopes, expectations, and concerns regarding PRISM.

Methods: Patients aged $<21$ years with a high-risk malignancy (anticipated survival rate $<30 \%$ ) are eligible for PRISM. Patients, parents and HCPs opt in to PRISMImpact and complete surveys upon enrolment (T0), after 
results delivery (T1), and then annually for five years (T25). HCP will be interviewed annually.

Results: To date, 17 families and 28 HCPs have opted in. Eleven parents ( 8 mothers) and three adolescents have returned surveys and $23 \mathrm{HCPs}$ were interviewed. A minority of parents expected a 'very likely'-'extremely likely' benefit from PRISM (4/11). At T0, most parents and adolescents expected that their child's chance of being cured would increase (7/11) and that they would learn something about their child's genes (9/11). Most parents indicated that they understood well what PRISM was about (6/11). Most parents also believed that PRISM would help find cures for future patients (10/11). Eighteen percent of HCPs believed that patients will benefit the most from PRISM and $40 \%$ of HCPs believed that future patients will benefit the most. Conclusion: At enrolment, families' hopes of a benefit are high. Through PRISM-Impact, we will identify barriers to, and predictors of, application of precision medicine trials into clinical practice. Our results are important for future trials across illnesses and age groups.

J. Vetsch: None. C.E. Wakefield: None. K. Tucker: None. M. Warby: None. P. Techakesari: None. G. Marshall: None. T.N. Trahair: None. T. O' Brien: None. J.M. Marron: None. L. Lau: None. V. Tyrell: None. D. Ziegler: None.

\section{EBPL2 The legal side of Genomic Care}

\section{EBPL2.1}

The evolving duty of care in clinical genomics under UK law

\section{A. E. Hall, J. Ordish}

\section{PHG Foundation, Cambridge, United Kingdom}

Technological changes coupled with new ways of working have created opportunities for new paradigms of treatment and care in clinical genomics. This presentation explores emergent challenges - specifically the extent to which existing duties of care might be modified or new duties created in the light of recent UK case law. The three cases which will be explored exemplify three foundational elements of genomics practice: consent [Montgomery (Appellant) $v$ Lanarkshire Health Board (Respondent) (Scotland) [2015]]; confidentiality [ABC v St George's Healthcare NHS Trust and Others (UK)[2017]] and the reporting of findings [Meadows v Khan [2017]].

The particular features that will be explored characterise the transition from genetics to genomics and include the widening scope of testing from targeted testing to whole exome sequencing/whole genome sequencing; the concomitant increase in generating, validating and reporting secondary findings, and increasing use of multidisciplinary teams involving new professional groups or existing professionals in novel ways. Together these highlight potential changes to the scope of the duty of care in clinical genomics (e.g. a potential duty to feedback secondary findings); by whom (e.g. by non-clinicians) and to whom such a duty might be owed (e.g. to genetic relatives).

Although the presentation will primarily focus on UK case law, this provides a precedent for a more general exploration of legal duties to patients and families and the aim will be to clarify how such risks might be ameliorated and inform the development of best practice.

A.E. Hall: A. Employment (full or part-time); Significant; PHG Foundation. J. Ordish: A. Employment (full or part-time); Significant; PHG Foundation.

\section{EBPL2.2}

Concerns about genetic discrimination after regulation: a qualitative study of the situation regarding BRCA and Huntington's disease in Belgium

\section{A. Wauters, I. Van Hoyweghen}

\section{KU Leuven, Leuven, Belgium}

Despite not having unequivocal evidence for genetic discrimination and laws that have installed prohibition of genetic discrimination, individuals confronting genetic disease still seem to be concerned. The aim of this study was to gain in-depth understanding of experiences and concerns of possible genetic discrimination. This paper presents an analysis of semi-structured interviews with 42 individuals who have, had or were at risk for breast- and ovarian cancer (BRCA) or Huntington's disease (HD) in Belgium. Our findings demonstrate that even after regulation, participants express concerns about possible genetic discrimination. These concerns relate to direct forms of genetic discrimination, as in the context of insurance and employment but concerns were also expressed about more subtle and indirect forms of genetic discrimination, e.g. in social relations, where individuals fear to be treated 'differently' and unfairly. Worries for these rather subtle forms of genetic discrimination are more difficult to protect by law. Our study further demonstrates how these concerns emerge at particular moments in life, how levels and forms of concerns are influenced by the specific genetic disorder, and how these concerns are grounded in previous discriminatory experiences (of others), stereotype thinking and being part of a family with a genetic illness. Furthermore, it has been shown that participants apply different strategies to cope with their concerns (e.g. selectively disclosing genetic information).

A. Wauters: None. I. Van Hoyweghen: None. 


\section{EBPL2.3}

Life insurance and genetic discrimination: A barrier for genomic medicine in Australia

\section{J. M. Tiller}

Public Health Genomics, School of Public Health and Preventive Medicine, Monash University, Melbourne, Australia

Introduction: Genetic discrimination by life-insurers remains an issue of international concern, with many European countries banning their use of genetic test results. The Australian situation, by contrast, is concerning and should noted by European countries as a reminder of the continued importance of this issue. Australian life-insurance companies can still use genetic test results to discriminate, with scant regulatory oversight[1]. As a result, Australians are now avoiding predictive gene testing, and participation in research, due to insurance fears[2-4]. The life-insurance industry is under scrutiny by a Parliamentary Inquiry[5], while Australia moves towards implementing genomic medicine[6, 7]. Regulatory reform is now overdue[8]. Genetics professionals play a key role.

Materials and Methods: On behalf of the Australian Genetic Non-Discrimination Working Group, we present evidence from a) official submissions to the Parliamentary Inquiry regarding genetic discrimination; b) results from a survey of over 85 Australasian clinical geneticists and genetic counsellors regarding professional practices and attitudes under current regulation; c) results from a survey of over 100 Australian cancer mutation carriers regarding experiences obtaining life-insurance.

Results: Findings show evidence of genetic discrimination by life-insurance companies in Australia, and identified considerable variability in professional practices and workforce training on the issue. The Parliamentary Inquiry will make its official recommendations for reform in March 2018, which we will update.

Conclusions: The Australian situation regarding genetic testing and life-insurance demonstrates the dangers of failing to regulate as many European countries have done and serves as a timely reminder of the importance of continuing vigilance regarding this issue.

J.M. Tiller: F. Consultant/Advisory Board; Significant; myDNA- legal consulting.

\section{EBPL2.4}

Working with the public: engaging with consumers about the ethics of and decision to pursue personal genomic testing
J. Savard ${ }^{1,2}$, C. Hickerton ${ }^{3}$, S. Metcalfe ${ }^{3,4}$, B. Terrill ${ }^{2,5}$, A. Newson ${ }^{1,2}$, C. Gaff ${ }^{4}$, K. Gray ${ }^{4}$, A. Middleton ${ }^{6}$, B. Wilson $^{7}$

${ }^{1}$ The University of Sydney, Camperdown, Australia, ${ }^{2}$ Garvan Institute of Medical Research, Darlinghurst, Australia, ${ }^{3}$ Murdoch Children's Research Institute, Melbourne, Australia, ${ }^{4}$ The University of Melbourne, Melbourne, Australia, ${ }^{5}$ The University of New South Wales, Randwick, Australia, ${ }^{6}$ Society and Ethics Research, Connecting Science, Welcome Genome Campus, Cambridge, United Kingdom, ${ }^{7}$ The University of Ottawa, Ottawa, ON, Canada

Personal genomic testing (PGT) for multiple purposes are marketed to ostensibly healthy people. Interest in these tests has significantly expanded in recent years, blurring boundaries between medical, 'wellness' and 'recreational' genomic testing.

As part of our Genioz project, in 2017 we conducted nine public deliberative workshops Australia-wide, involving 129 participants with $(n=23)$ and without $(n=106)$ experience of testing. We aimed to explore non-expert perspectives on PGT. Drawing on earlier survey and interview data, we selected three categories of testing for exploration: ancestry $(n=61)$, personal wellness $(n=34)$, and children's health and aptitudes $(\mathrm{n}=34)$.

Four stepwise, neutrally-framed information sets were presented pertaining to key scientific and ethical issues in PGT, with time for facilitated discussion. Data were captured using mixed methods approach. We report the results of: tracker questions from 5 time points in the workshops; participant attitudes regarding information consumers should know about personal genomic tests; and a thematic analysis of discussions and written responses by participants.

Tracker question data registered trends of participants becoming less comfortable with and more thoughtful about pursuing testing. Discussions and written responses reflected this change, with participants identifying ethically complex issues (about consent, family communication and privacy) that aligned with information they rated as important to know when considering PGT.

This published deliberative methodology highlights a useful public engagement strategy, demonstrating that nonexperts can contribute to meaningful conversations about the decision to pursue PGT. These findings can inform policy and resources to help people critically reflect so they can make informed decisions around PGT.

J. Savard: None. C. Hickerton: None. S. Metcalfe: None. B. Terrill: Other; Modest; Genome.One. A. Newson: None. C. Gaff: None. K. Gray: None. A. Middleton: None. B. Wilson: None. 
EPL1 Ensuring good clinical practice in whole genome sequencing

\section{EPL1.1}

Knowledge about and attitudes towards whole-genome sequencing among participants in the 100,000 Genomes Project: a multi-site survey

\section{S. C. Sanderson, C. Lewis, L. C. Chitty}

\section{Great Ormond Street Hospital, London, United Kingdom}

Introduction: Patients with rare diseases and their relatives will increasingly be offered diagnostic whole-genome sequencing (WGS). Our aim was to examine knowledge and attitudes among families who had been offered WGS.

Materials and Methods: A questionnaire was administered to patients and relatives $(n=220)$ who had been invited to take part in the 100,000 Genomes Project. The survey instrument included the 9-item Knowledge of Genome Sequencing (KOGS) scale and a 14-item attitudes measure, both of which were newly developed for this study.

Results: Ninety-four (43\%) participants were patients with rare diseases, 126 (57\%) parents/relatives; 62\% female; mean age 45yrs (range 16-79yrs); $48 \%$ had a degree; $83 \%$ White British. The mean KOGS score was $5.05(0=$ low knowledge, $9=$ high knowledge $)$. On individual items, most $(85.5 \%)$ understood that "a person's genome is their body's 'instruction manual' containing the information needed to make them, run them and repair them." Fewer $(57.7 \%)$ understood that "there are uncertainties about what a person's genome can tell them." In terms of attitudes, most believed WGS could identify the underlying cause of their condition $(89.5 \%)$ and advance medical research $(99.5 \%)$. Some worried about insurance companies $(20.5 \%)$ and commercial companies $(13.2 \%)$ having access to their health information; more $(29.1 \%)$ were worried about how they would feel if they learned they had a high risk of developing a serious disease. Few differences between socio-demographic groups were detected.

Conclusions: These findings may be of value to researchers, clinicians and policy-makers involved in developing WGS protocols and information materials for patients and their families.

S.C. Sanderson: None. C. Lewis: None. L.C. Chitty: None.

\section{EPL1.2}

A clinician survey: diagnostic utility, impact on patient management, and outcomes of clinical exome sequencing

\section{K. McWalter, M. Cho, J. Juusola}

GeneDx, Gaithersburg, MD, United States

Introduction: Exome sequencing (ES) is often ordered on patients with complex phenotypes and non-diagnostic prior testing. We surveyed clinicians to determine how ES is utilized, its impact on medical management, and overall outcomes.

Methods: Participants completed either two IRBapproved prospective surveys (four-weeks and one-year after ES report) or one retrospective survey (one-year after ES report). Surveys examined demographics, results interpretation, and medical management following ES.

Results: Of 1064 surveys sent, 249 were completed (139 prospective, 98 retrospective, 12 follow-up); $23 \%$ response rate. Respondents were mostly female (64\%), Caucasian (89\%), either physicians $(64 \%)$ or genetic counselors $(34 \%)$, and ordered $>3$ ES tests/month (76\%). Prior testing included microarray, imaging, and metabolic screening. Of 163/249 cases with reportable variants, $48 \%$ of clinicians said result explained patient's phenotype, $33 \%$ were uncertain, and $19 \%$ thought it did not explain the phenotype. In $42 \%$ of cases, a molecular diagnosis was established via ES. In $27 \%$ of inpatient cases (14/51), ES result impacted length of hospitalization. In $34 \%$ of cases, the ES result clarified the patient's prognosis. Overall, participants rated clinical usefulness of ES testing 80/100. In select families, 69\% of clinicians said the ES result impacted family planning. After ES result, $27 \%$ of retrospective families found a family support network, 33\% contacted research groups, $29 \%$ joined research studies, and 10\% participated in clinical trials.

Conclusion: Clinicians rated ES clinical utility highly and used results to guide medical management. ES impacted hospitalization times, clarified prognoses, family planning, and establishing family support.

K. McWalter: A. Employment (full or part-time); Significant; GeneDx. M. Cho: A. Employment (full or part-time); Significant; GeneDx. J. Juusola: A. Employment (full or part-time); Significant; GeneDx.

\section{EPL1.3}

Motivations and Barriers for participants and decliners of the 100,000 Genomes Project from different ethnic backgrounds

\section{N. Chauhan ${ }^{1,2}$, S. Ellis ${ }^{1,3}$, K. Tricker ${ }^{4}$, W. Newman ${ }^{1,4}$,} B. Starling $4^{5}$, G. Hall,

${ }^{1}$ University of Manchester, Manchester, United Kingdom, ${ }^{2}$ Oxford University Hospitals NHS Foundation Trust, Oxford, United Kingdom, ${ }^{3}$ NHS Greater Glasgow and Clyde, Glasgow, United Kingdom, ${ }^{4}$ Central Manchester University Hospitals, Manchester, United Kingdom, ${ }^{5}$ Wellcome Trust Manchester, Manchester, United Kingdom 
Introduction: Using genomic testing in medicine is increasingly establishing a role in clinical services. The 100,000 Genomes Project (100kGP) is offering Whole Genome Sequencing (WGS) to patients in centres across the U.K. Our research aim is to explore the motivations and barriers faced by people from different ethnic backgrounds and decliners of this project.

Method: 14 people were interviewed for this study, 13 participants and 1 decliner; 8-White British, 4-Asian, 1Mixed race and 1- Unknown. This qualitative research utilised semi-structured interviews, conducted face-to-face or on the telephone. Data was analysed using Interpretative Phenomenological Analysis.

Results: Our participants have often experienced a previous 'diagnostic odyssey'. Most were motivated to participate to potentially gain a genetic diagnosis or to help others if this wasn't personally attainable. Participants also shared some barriers to participation; a link between nonnative language and trust, impact of cultural and religious beliefs on decision making; lack of education and awareness, 'bad' timing' and an overwhelming amount of information received.

Conclusions: Very little is known about why we see fewer people from minority groups utilising genetic services in the UK. This also applies to the views of those who declined to participate in this kind of research. Our study provides an insight into the needs of participants, which can be utilised to support potential participants to gain access to genetic services. The findings from this study form a strong base for future research and outline valuable clinical recommendations that could be used to motivate the uptake of genetic services.

N. Chauhan: None. S. Ellis: None. K. Tricker: None. W. Newman: None. B. Starling: None. G. Hall: None.

\section{EPL1.4}

\section{Patient perspectives after genomic sequencing testing in clinical care}

\section{Martyn ${ }^{1,2,3}$, A. Kanga-Parabia ${ }^{1,3}$, L. Keogh $^{3}$, A. Trainer ${ }^{4,5}$, Melbourne Genomics Health Alliance Community Advisory Group, C. L. Gaff ${ }^{1,3}$}

${ }^{I}$ Melbourne Genomics Health Alliance, Parkville, VIC, Australia, ${ }^{2}$ Murdoch Children's Research Institute, Parkville, VIC, Australia, ${ }^{3}$ The University of Melbourne, Parkville, VIC, Australia, ${ }^{4}$ Peter MacCallum Cancer Centre, Parkville, VIC, Australia, ${ }^{5}$ Melbourne Health, Parkville, VIC, Australia

Studies of clinical utility have demonstrated the medical impact of genomic sequencing tests on the management of patients with germline or somatic disorders. Here, we report results of the first pre- and post-test study investigating aspects of patient and familial utility. We administered surveys to patients receiving either germline or somatic genomic sequencing as part of their clinical care pathway. To date, response rates are $70 \%(566 / 805)$ at baseline and $48 \%(116 / 240)$ at follow-up, with $62 \%(72 / 116)$ responding at follow-up for themselves and 38\% (44/116) as parents of a child. Although all (47/47) with a diagnosis or actionable mutation correctly recalled their result, 8/26 (31\%) cancer patients and 3/35 (9\%) germline patients with an uninformative result were unsure or incorrect. Those who obtained a diagnosis/actionable mutation had a significant increase in perceived personal control (empowerment) (1.064 v 1.463, $\mathrm{p}<0.001)$, but this was not evident for those people who did not (1.064 v 0.989, $\mathrm{p}=0.3$ ). Interestingly, results with clinical utility may be not always be viewed positively by patients. 7/104 (7\%) participants exhibited decision regret, including 3 cancer patients with clinically actionable findings. Furthermore, 17 patients reported unanticipated consequences, with two who did not obtain a diagnosis surprised this was not the end of investigations. Differences between test outcomes valued by patients were evident across the 11 clinical subgroups and will be detailed. Though generally positive, our results shed light on those who may need greater attention and contribute to debate about measurement of patient outcomes in genomics.

M. Martyn: None. A. Kanga-Parabia: None. L. Keogh: None. A. Trainer: None. C.L. Gaff: None.

\section{EPL1.5}

Reproductive and heteronormative presumptions in disclosure of pediatric whole exome sequencing results

\section{A. Werner-Lin, S. Merrill, R. Mueller, M. Carlson, L. Zaspel, S. Walser, S. Biswas, B. Bernhardt}

University of Pennsylvania, Philadelphia, PA, United States

Introduction: Whole exome sequencing (WES) can identify variants associated with future reproductive risk. Though fully informed reproductive decision-making is of potential benefit, research has not explored the potential negative impact on autonomy or self-esteem, particularly in pediatric settings. Consideration of how reproductively relevant pediatric WES results are relayed and received is critical to inform best practices.

Methods: Adolescents with hearing loss, personal/family history of cardiac conditions, and platelet disorders completed WES for a research protocol. Beyond diagnostic results, families could opt to receive incidental findings for medically actionable childhood disorders, adult onset disorders, and/or carrier status. An interdisciplinary team analyzed audiofiles of 10 results disclosure sessions paired with three-month followup interviews with parents and patients. 
Findings: Regardless of patient age, providers implicitly and explicitly conveyed that WES findings, primarily carrier status results, necessitated active family planning. At follow-up, parents and adolescents repeated these messages. In all sessions, provider language revealed assumptions that adolescents planned on both marriage to opposite-sex partners and biological parenthood. Providers counseled, and adolescents repeated, pre-conception testing of future partners and, in one case, pre-implantation genetic diagnosis, to prevent the birth of an affected child. No provider queried patient sexual orientation or intent to partner or reproduce.

Conclusions: Providers suggested adolescents prevent the birth of an affected child by avoiding unplanned pregnancy through utilization of prenatal testing and/or reproductive technologies. These messages implicitly challenged adolescents' self-worth during a critical period of identity development and challenged future reproductive choice by introducing moral imperatives around responsible genomic citizenship.

A. Werner-Lin: A. Employment (full or part-time); Significant; University of Pennsylvania. B. Research Grant (principal investigator, collaborator or consultant and pending grants as well as grants already received); Significant; National Institutes of Health. S. Merrill: None. R. Mueller: None. M. Carlson: None. L. Zaspel: None. S. Walser: None. S. Biswas: None. B. Bernhardt: B. Research Grant (principal investigator, collaborator or consultant and pending grants as well as grants already received); Significant; National Institutes of Health.

\section{EPL1.6}

Facilitating understanding of whole genome sequencing in young people

\section{Lewis ${ }^{1,2}$, S. C. Sanderson ${ }^{1,2}$, L. S. Chitty ${ }^{1,2}$}

${ }^{1}$ North East Thames Regional Genetics Service, Great Ormond Street Hospital for Children NHS Foundation Trust, London, United Kingdom, ${ }^{2}$ Genetics and Genomic Medicine, The UCL Great Ormond Street Institute of Child Health, London, United Kingdom

Introduction: Young people with rare diseases are increasingly having whole-genome sequencing for diagnostic purposes. To make informed decisions, they need adequate knowledge, but suitable decision-support tools are lacking, as are relevant knowledge measures. Young people in the general public also need adequate knowledge to engage in public discourse. The aims of this study were to develop a new educational tool and knowledge measure about whole-genome sequencing for use with young people, and to evaluate the impact of the intervention.
Materials and Methods: With input from young people we developed a 2.5 minute animation about whole-genome sequencing, "My Genome Sequence," which is freely available on YouTube (https://goo.gl/ogkzYA). To develop the new 10-item measure of Young People's Knowledge of Genome Sequencing (the "Kids-KOGS") we conducted qualitative interviews, reviewed 100,000 Genomes Project patient information and consent documents and other published literature. We piloted the measure with young people to ensure readability. Finally, we showed the animation to young people $(n=417)$ aged $11-17 \mathrm{yrs}$ at three UK schools. Participants completed the Kids-KOGS and a subjective knowledge measure before and after watching the animation.

Results: Objective knowledge was higher after viewing the animation: mean Kids-KOGS scores increased from 4.68 to $7.88(\mathrm{t}=26.52, \mathrm{p}=0.000009)$, where $0=$ low and $10=$ high knowledge. Mean subjective knowledge scores also increased, from 2.01 to 2.27 (possible range 1-3) $(\mathrm{t}=$ 9.86, $\left.\mathrm{p}=1.06 \times 10^{-20}\right)$.

Conclusion: We have developed a 2.5 minute animation and 10-item knowledge measure that may be valuable tools for healthcare providers and researchers working with young people in the area of whole-genome sequencing.

C. Lewis: None. S.C. Sanderson: None. L.S. Chitty: None.

\section{EPL2 Improving communication in genetic counselling}

\section{EPL2.1}

'Music of Life' a new metaphor for genomics, delivered as film within genetic counselling

A. Middleton ${ }^{1}$, J. Borra ${ }^{2}$, T. Pope ${ }^{3}$, V. Wiles ${ }^{4}$, J. Roberts ${ }^{1}$, L. Farley ${ }^{1}$, I. Turbin ${ }^{4}$, A. O'Shaughnessy-Kirwan ${ }^{4}$, F. Wadrup ${ }^{4}$, S. Kenwrick ${ }^{4}$, B. Speight ${ }^{4}$, E. Davies ${ }^{4}$, J. Matthews-Kelly ${ }^{4}$,

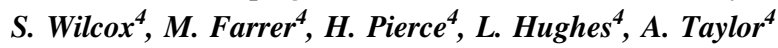

${ }^{1}$ Connecting Science, Wellcome Genome Campus, Cambridge, United Kingdom, ${ }^{2}$ Thin Air Factory, London, United Kingdom, ${ }^{3}$ Black Label Productions, London, United Kingdom, ${ }^{4}$ Addenbrooke's Hospital, Cambridge, United Kingdom

Introduction: The metaphors currently used within genetic counselling practice to describe genetics concepts have very little evidence that underpin their use. Using creative story telling expertise and cutting-edge film making, we have made a series of films that aim to socialise genomics for patients; these will be delivered and evaluated within genetic counselling practice.

Materials and Methods: An iterative series of 4 workshops were conducted with the team of 13 genetic 
counsellors from Addenbrooke's Hospital Cambridge. Led by an expert from the creative storytelling industry (collaborator: Thin Air Factory), we gathered feedback from genetic counselling experts on their use of existing metaphors and explored the development of a new metaphor about genomics that they perceived would cross cultures, span generations and be meaningful to all sociodemographic groups of patients.

Results: The 'music of life' concept emerged through an inductive process of discussion and debate. The concept: Song $=$ Gene, Notes in a song $=$ DNA, Album $=$ Chromosome. Changes to the notes within a song could represent the variation between us. This metaphor has been used as the basis to create 6, $90 \mathrm{sec}$ films: 'what is gene?', 'recessive', 'dominant', 'X-linked', 'de novo' and 'outcomes from a diagnostic test'. We will show the films and present preliminary findings from their delivery and evaluation within genetic counselling practice.

Conclusions: We have found a new metaphor to describe genomics with patients.

Grant: Translation Fund, Biodata Innovation Centre, Wellcome Genome Campus, Cambridge

A. Middleton: None. J. Borra: A. Employment (full or part-time); Significant; Thin Air Factory Founder. T. Pope: A. Employment (full or part-time); Significant; Black Label Productions Film Director. V. Wiles: None. J. Roberts: None. L. Farley: None. I. Turbin: None. A. O'Shaughnessy-Kirwan: None. F. Wadrup: None. S. Kenwrick: None. B. Speight: None. E. Davies: None. J. MatthewsKelly: None. S. Wilcox: None. M. Farrer: None. H. Pierce: None. L. Hughes: None. A. Taylor: None.

\section{EPL2.2}

A large outcome study on genetic counseling in the Netherlands: empowerment and emotional functioning

J. S. Voorwinden ${ }^{1}$, M. Plantinga ${ }^{1}$, M. Ausems ${ }^{2}$, N. Knoers ${ }^{2}$, M. Velthuizen ${ }^{2}$, E. Birnie ${ }^{1}$, A. M. Lucassen ${ }^{3}$, A. V. Ranchor ${ }^{1}$, I. M. van Langen ${ }^{1}$

${ }^{1} U M C G$, Groningen, Netherlands, ${ }^{2} U M C U$, Utrecht, Netherlands, ${ }^{3}$ University of Southampton, Southampton, United Kingdom

Introduction: More evidence based research to understand how genetic counseling is experienced by counselees, as compared to the goals/objectives of counseling viewed by its providers, is needed. The aim of this study is to measure these outcomes including empowerment and emotional functioning for a large and diverse Dutch study sample and associate these with socio-demographic and clinical variables.
Materials and Methods: Data was collected with validated questionnaires from 2502 counselees on three different time-points: before genetic counseling started (T0), after the intake (T1) and after the results were communicated (T2). Questionnaires were the GCOS-18 (empowerment and different facets), PPC (perceived personal control), STAI (state anxiety) and PANAS (positive and negative emotions).

Results: Compared to T0, there were significant but small improvements in most outcome measures on $\mathrm{T} 1$ and $\mathrm{T} 2$. Exceptions were a moderate increase in empowerment $(\mathrm{d}=.52)$ and a large decrease in uncertainty about heredity $(\mathrm{d}=.67)$. Regarding subgroups, outcomes of genetic counseling were significantly associated with referral reason, type of disease and genetic test result, while socio-demographic variables were not. Conclusion: Counselees in our sample experienced the strongest gains in empowerment, most explained by decreased uncertainty about heredity. Other facets of empowerment (like knowledge, hope, negative emotions) and other outcome measures (like state anxiety) changed less. This study provides more insight in the black box of genetic counseling and its desired outcomes plus which subgroups profit more and where improvements are possible.

J.S. Voorwinden: None. M. Plantinga: None. M. Ausems: None. N. Knoers: None. M. Velthuizen: None. E. Birnie: None. A.M. Lucassen: None. A.V. Ranchor: None. I.M. van Langen: None.

\section{EPL2.3}

Improving communication for individuals with a rare condition

\section{A. L. Crowe, H. McAneney, J. C. McMullan, A. McKnight}

Centre for Public Health, Queen's University Belfast c/o Regional Genetics Centre, Level A, Tower Block, Belfast City Hospital, Lisburn Road, BT9 7AB, Belfast, United Kingdom

Introduction: One in 17 individuals in the UK are affected by a rare condition, with $\sim 80 \%$ having a genetic cause. Major local problems include a lack of accurate diagnosis, no clarity about specialist care, a lack of coordinated approach, medical professionals who have not heard of the disease, and challenges sourcing relevant therapies. Alongside our newly implemented whole genome sequencing programme to improve rare disease diagnosis, improving complementary communication mechanisms in the health and social care system is of vital importance.

Materials and Methods: A review of existing information and communication resources for individuals living and working with rare diseases was held in Northern Ireland between January 2017 and February 2018. Strategies 
included three surveys, five public consultation meetings, and individual interviews with a range of stakeholders. Using the DELPHI model, priorities for improving communication were identified and consensus achieved.

Results: More than 500 individuals engaged with the process and contributed to identifying $>60$ priority needs, alongside evaluating strengths/barriers to implementing improved communication strategies. Identified priorities were categorised under the following four headings: (i) sources of information, (ii) medical care, (iii) rare disease community, and (iv) public awareness.

Conclusions: Developing an effective information hub for rare diseases in Northern Ireland was the top-ranked strategic priority from all individual and collective information procurement strategies.

ALC is supported by a $\mathrm{PhD}$ studentship from the Department for the Economy. This work was conducted in collaboration with the Northern Ireland Department of Health, the Public Health Agency, and the Northern Ireland Rare Disease Partnership.

A.L. Crowe: None. H. McAneney: None. J.C. McMullan: None. A. McKnight: None.

\section{EPL2.4}

Empowering service users: Assessing the potential benefits of psychiatric genetic counselling from the 1st UK pilot study

\section{S. Watson ${ }^{1}$, K. A. McGhee ${ }^{2}$, J. C. Austin ${ }^{3}$}

${ }^{1}$ Wessex Clinical Genetics Service, Southampton, United Kingdom, ${ }^{2}$ Bournemouth University, Bournemouth, United Kingdom, ${ }^{3}$ University Of British Columbia, Southampton, BC, Canada

Introduction: Psychiatric Genetic Counselling (PGC) is an emerging specialty within the genetic counselling profession. The world's first specialist PGC service was established in Vancouver 2012, providing genetic counselling to individuals with mental illness and their families. Psychiatric disorders have both a genetic and non-genetic aetiology. This study aimed to ascertain whether British patients with mental illness would welcome such a service and after PGC report an increase in self-efficacy, empowerment over their illness and a reduction in stigma.

Materials and Methods: Patients $(n=10)$ with a diagnosis of schizophrenia, bipolar or depression, under the care of Dorset Healthcare University Foundation Trust were invited by their psychiatrists to participate in a genetic counselling session, led by a genetic counsellor (Wessex Regional Genetics Service). The session was a clinical intervention similar to that offered for a referral for any disease with a hereditary component, but specific to psychiatry. Each patient was asked to complete the Genetic Counselling Outcome Scale (GCOS-24), prior to a one-hour genetic counselling session. Patients were then asked to complete the GCOS-24 at a four week (post counselling) follow up telephone appointment.

Results: Participants reported a significant increase in self efficacy and empowerment over their illness post this genetic counselling intervention.

Conclusions: In line with previous data (Austin \& Honer 2008; Hippman et al. 2016) this study demonstrates that genetic counselling has a positive impact on this patient group and is considered both an acceptable and useful addition to their usual psychiatric care .

M.S. Watson: None. K.A. McGhee: None. J.C. Austin: None.

\section{EPL2.5}

Bridging the gaps of uncertainty in genetic counselling with ethnic-specific data

Y. M. Bylstra ${ }^{1,2}$, J. Kuan ${ }^{1,3}$, W. Lim ${ }^{1,4}$, B. Digambar ${ }^{5}$, J. Teo ${ }^{1}$,

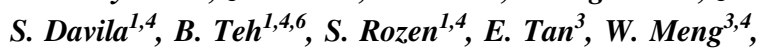
K. Yeo ${ }^{5}$, P. Tan ${ }^{1,4,7}$, S. Saw ${ }^{8,9,4}$, C. Cheng ${ }^{9,4}$, S. Cook ${ }^{1,5,4}$, R. Foo $^{8,4}$, S. Jamuar ${ }^{1,3,4}$

${ }^{1}$ Singhealth Duke-NUS Institute of Precision Medicine, Singapore, Singapore, ${ }^{2}$ National University Hospital, Singapore, Singapore, ${ }^{3} \mathrm{KK}$ Women's and Children's Hospital, Singapore, Singapore, ${ }^{4}$ Duke-NUS Medical School, Singapore, Singapore, ${ }^{5}$ National Heart Centre Singapore, Singapore, Singapore, ${ }^{6}$ National Cancer Centre, Singapore, Singapore, ${ }^{7}$ Agency for Science, Technology and Research, Singapore, Singapore, ${ }^{8}$ National University of Singapore, Singapore, Singapore, ${ }^{9}$ Singapore National Eye Centre, Singapore, Singapore

Uncertainty is intrinsic to genetic counselling and whilst genomic sequencing is valuable in medical diagnosis, it also brings uncertainty regarding clinical utility. Human genomic variant databases assist with variant interpretation however they contain information derived largely from European origin. In under-served populations, the challenges experienced in understanding genomic variant significance are further pronounced. The SingHealth DukeNUS Institute of Precision Medicine (PRISM) was established to promote precision medicine for medical conditions relevant to South East Asian populations, currently an under-represented cohort. To identify disease-causing variants within the Singaporean population, existing exome and genome sequencing data of 831 Singaporeans with no known pre-existing health conditions were aggregated. This evolving dataset serves as a valuable reference for understanding genomic variant significance for the Singaporean 
population. In the first instance, we chose to define the prevalence of 80 treatable inherited disorders (TIDs). Genomic variant filtering and analysis identified 1 in 18 individuals $(6 \%)$ to be carriers of one of 13 treatable causes of intellectual disability. Citrin deficiency and Wilson disease had the highest carrier frequency of 1 in 41, and 1 in 103 individuals, respectively. In addition, the pathogenic variants associated with citrin deficiency were 24 times more prevalent in our local cohorts when compared to Western cohorts. The development of this framework has enabled the discovery of novel pathogenic variants to optimise diagnosis and carrier risk assessment in genetic counselling practice. This research adds further evidence that ethnic-specific data is necessary for variant interpretation and genetic counselling.

Y.M. Bylstra: None. J. Kuan: None. W. Lim: None. B. Digambar: None. J. Teo: None. S. Davila: None. B. Teh: None. S. Rozen: None. E. Tan: None. W. Meng: None. K. Yeo: None. P. Tan: None. S. Saw: None. C. Cheng: None. S. Cook: None. R. Foo: None. S. Jamuar: None.

\section{EPL2.6}

myKinMatters: developing a web app tool to help patients create a family tree and communicate genetic health information to at-risk relatives

\section{M. Ballard ${ }^{1}$, S. Dheensa ${ }^{2,1}$, M. Weal ${ }^{3}$, A. Fenwick ${ }^{1}$, A. Lucassen ${ }^{1}$}

${ }^{1}$ Clinical Ethics and Law, University of Southampton, Southampton, United Kingdom, ${ }^{2}$ Cardiff School of Social Sciences, Cardiff University, Cardiff, United Kingdom, ${ }^{3}$ Web Science Institute, University of Southampton, Southampton, United Kingdom

Introduction: The result of an individual's genomic test can clarify risks for close relatives, but research has shown that patients do not always communicate their results to those to whom it is relevant. Research suggests that certain groups of patients want more information and help through online sources. Our aim was to increase rates of communication by developing a Web app tool, making sharing genetic information easier.

Methods: A prototype Web app tool was built using open source software frameworks (Laravel 5, MySQL), which allowed patients to build a family tree and upload test results to share with at-risk relatives. This tool has been designed to be embedded in an existing eHealth record (My Medical Record).

Results: myKinMatters enables: patients and health professionals to draw a family tree highlighting who has a condition and who might be at risk; patients to upload test results and share these with relatives with accompanying messages/interpretations; and has a mixed media section containing 'tips' for communicating with relatives.

Discussion: We have previously demonstrated that current methods employed to alert relatives miss a significant group of at-risk relatives. myKinMatters could provide a useful additional tool for clinicians to offer patients and will facilitate relatives to make informed decisions about their own testing, screening programmes, or preventative treatment. We are now testing the usability and acceptability of the app with patients who recently received a genetic test result and specialist and non-specialist health professionals.

Grant information: Wessex Medical Research Innovation Fund was awarded to Dr Dheensa.

L.M. Ballard: None. S. Dheensa: None. M. Weal: None. A. Fenwick: None. A. Lucassen: None.

\section{EPL3 Educating Professionals and Public}

\section{EPL3.1}

'What is genomics as I've never heard of it?': The challenges of identifying the education needs around an emerging clinical area

\section{Bishop ${ }^{1}$, S. Simpson ${ }^{1}$, H. Brown ${ }^{2}$, Y. Sawbridge ${ }^{2}$, A. Seller ${ }^{1}$ \\ ${ }^{1}$ Genomics Education Programme, Edgbaston, United Kingdom, ${ }^{2}$ University of Birmingham, Edgbaston, United Kingdom}

In October 2018, a new genomic medicine service in England's National Health Service (NHS) will launch, bringing with it a need for education and training across the 1.3 million workforce. Health Education England's Genomics Education Programme has taken two approaches to identify the educational needs across this large and diverse group to inform our development priorities. First, we surveyed, via our network in the Genomic Medicine Centres, 2,578 NHS employees, representing all professional groups. While the results did reveal knowledge and skill gaps, it was difficult to determine clear themes for content owing to the broad variety of responses. In addition, many professionals stated they had no or limited knowledge of genomics and as such couldn't define the education or training they'd require. Secondly, we conducted an exploratory study focused on two patient pathways where genomics is integrated into the patient pathway (ovarian cancer and familial hypercholesterolaemia). Contact-point mapping was undertaken to identify the professionals involved, and 19 individuals were interviewed. Transcripts were analysed using thematic analysis, revealing three key themes: for content to focus on skills (e.g. communication) rather than scientific 
knowledge; to educate within a multidisciplinary environment to help staff consider their role in the context of the patient pathway; and to ensure content is directly relevant to individuals' practice. The findings from these studies have highlighted the complexity of identifying the educational needs of such a broad group, and demonstrate the importance of integrating data from multiple approaches to build a picture of the genomics education landscape.

M. Bishop: None. S. Simpson: None. H. Brown: None. Y. Sawbridge: None. A. Seller: None.

\section{EPL3.2}

Genetic counsellors' clinical practice in Europe: a mixed method assessment/approach on their contribution

\section{Ingvoldstad ${ }^{1,2,3}$, C. Cordier ${ }^{4}$, R. Moldovan ${ }^{5}$, M. Paneque ${ }^{6,7}$, R. Pestoff ${ }^{8,9}$, C. Serra-Juhe $e^{10,11}$}

${ }^{1}$ Dept of Clinical Science, Intervention and Technology, Division of Obstetrics and Gynaecology, Stockholm, Sweden, ${ }^{2}$ Department of Public Health and Caring Science, Uppsala University, Uppsala, Sweden, ${ }^{3}$ Department of Women's and children's health, Uppsala University, Uppsala, Sweden, ${ }^{4}$ Synlab Genetics, Department of Genetics, Lausanne, Switzerland, ${ }^{5}$ Department of Psychology, Babeş-Bolyai University, Cluj-Napoca, Romania, ${ }^{6}$ i3S Instituto de Investigação e Inovação em Saúde, Universidade do Porto, Porto, Portugal, ${ }^{7}$ Centre for Predictive and Preventive Genetics CGPP-IBMC Institute for Molecular and Cell Biology Universidade do Porto, Porto, Portugal, ${ }^{8}$ Department of Clinical Genetics, Linköping University Hospital, Linköping, Sweden, ${ }^{9}$ Department of Clinical and Experimental Medicine, Linköping University, Linköping, Sweden, ${ }^{10}$ Genetics Unit, Universitat Pompeu Fabra - Hospital del Mar Research, Barcelona, Spain, ${ }^{11}$ Centro de Investigación Biomédica en Red de Enfermedades Raras (CIBERER), Instituto de Salud Carlos III, Madrid, Spain

Non-medical healthcare professionals are integrated in genetic services everywhere. Nevertheless, genetic counsellors' roles differ without a unified approach in healthcare services in Europe. The profession is still emerging in many countries and the educational backgrounds diverge noticeably. This mixed methods study combined a qualitative and quantitative approach and aimed to explore tasks and responsibilities in Europe, and identify potential added value of genetic counsellors in clinical genetics teams. Our study also explored whether and to what extent genetic counsellors' characteristics have an impact in practice, and which tasks are most relevant for genetic counsellors, according to genetic counsellors themselves and according to medical geneticist colleagues. A total of 143 participants (104 GC; $29 \mathrm{MD})$ providing genetic counselling in 15 countries in Europe responded. Results showed that most genetic counsellors in Europe perform similar tasks, irrespective of their backgrounds. The main factor influencing what tasks genetic counsellors' do was the years of experience in the field, not background nor education. Genetic counsellors and medical geneticists agreed regarding the genetic counsellor's role. Tasks with psychosocial implications were mainly seen as genetic counsellors' role and tasks with medical implications were seen as medical geneticists' attribution. Our study shows that most genetic counsellors work in tune with international recommendations and support working in multidisciplinary teams. Finally, we discuss potential implications for practice and training in genetic counselling.

C. Ingvoldstad: None. C. Cordier: None. R. Moldovan: None. M. Paneque: None. R. Pestoff: None. C. Serra-Juhé: None.

\section{EPL3.3}

Clinical Genetics Educational external assessment (EQA)assuring improvement in the Clinical Service

\section{R. H. Hastings ${ }^{1}$, C. van Ravenswaaij-Arts ${ }^{2}$, C. van Asperen ${ }^{3}$, L. Garavelli ${ }^{4}$, M. Neilsen ${ }^{3}$, B. Peterlin ${ }^{5}, K$. Writzl $^{5}$ \\ ${ }^{1}$ Oxford University Hospitals NHS Foundation Trust, Oxford, United Kingdom, ${ }^{2}$ Dept of Clinical Genetics, Faculty of Medical Sciences, Groningen, Netherlands, ${ }^{3}$ Dept of Clinical Genetics, Leiden, Netherlands, ${ }^{4}$ Dept of Clinical Genetics, reggio emilia, Italy, ${ }^{5}$ Dept of Clinical Genetics, Ljubjiana, Slovenia}

Quality assessment has been associated with laboratory, but not clinical services. To address this gap, CEQAS was invited by the ESHG Eurogentest Quality sub-committee to provide an Educational EQA for clinical genetics services. A clinical working group advises CEQAS on the EQA format and content as well as preparing four case scenarios in the fields of cardiogenetics, oncogenetics, monogenetic disorders and dysmorphology. Each scenario starts with a referral letter and consists of multiple stages, to reflect an episode of clinical care. At each stage further clinical and genetic test information is given and a number of questions presented. For each EQA, consensus answers were provided by at least two clinical geneticists and a patient organisation was consulted. Sixteen EQAs have been distributed over four years to 57 genetic centres from 25 countries. All EQA submissions were reviewed by two assessors per case. The learning objectives are included in the summary letter. The answers provided by the centres highlighted differences in clinical genetics practice. The EQA identified that some centres omitted to include the need for: (a) consent of the index patient; (b) a three generation family history to be 
taken; (c) discussion of the implications of a recessive disorder; (d) comprehensive reproductive options and (e) psychosocial support for the patient and family. The EQA for clinical genetics is both feasible and highly educational showing improvement in the comprehensiveness of the answers given. The EQA cases, genetic consultation issues and proposals for a future Genetic Counselling pilot EQA will be presented.

R.H. Hastings: None. C. van Ravenswaaij-Arts: None. C. van Asperen: None. L. Garavelli: None. M. Neilsen: None. B. Peterlin: None. K. Writzl: None.

\section{EPL3.4}

The changing clinical practice of genomic medicine: what are the preferences of and education/training needs of health professionals?

B. McClaren ${ }^{1,2}$, Z. Prichard ${ }^{2,3}$, A. E. Nisselle ${ }^{1,2}$, E. Crellin ${ }^{1,2}$, M. Janinski ${ }^{1,2}, L_{.} \mathrm{Ng}^{4,2}$, E. Zilliacus $^{3}$, K. Dunlop $^{3,2}$, D. Graves $^{5,2}$, B. Terrill ${ }^{6,2}, A$. Newson ${ }^{7,2}$, C. Gaff ${ }^{8,2}$, S. A. Metcalfe ${ }^{1,2}$

${ }^{1}$ Murdoch Childrens Research Institute and Dept Paediatrics, University of Melbourne, Parkville, Vic, Australia, ${ }^{2}$ Australian Genomics Health Alliance, Parkville, Vic, Australia, ${ }^{3}$ Centre for Genetics Education, Sydney, NSW, Australia, ${ }^{4}$ Murdoch Childrens Research Institute, Parkville, Vic, Australia, ${ }^{5}$ The Royal College of Pathologists of Australasia, Sydney, NSW, Australia, ${ }^{6}$ Kinghorn Centre for Clinical Genomics, Garvan Institute of Medical Research, Sydney, NSW, Australia, ${ }^{7}$ Centre for Values, Ethics and the Law in Medicine, Sydney School of Public Health, The University of Sydney, Sydney, NSW, Australia, ${ }^{8}$ Walter and Eliza Hall Institute for Medical Research, Parkville, Vic, Australia

Successful implementation of genomics in healthcare requires well-informed, engaged health professionals (HPs) to request, identify and/or refer patients for genomic testing. This study describes current practice and identifies future genomic education/training needs of Australian health professionals. National data were collected in semistructured interviews. HPs were recruited through professional networks and medical Colleges; providers of recent education activities were contacted directly. Interviews were audio-recorded, transcribed and analysed using: deductive content analysis to document current education activities; and inductive coding to compare and contrast participant perspectives. HPs $(\mathrm{n}=81)$ and education providers $(\mathrm{n}=32)$ were interviewed. Clinical HPs reported varied practice in requesting genomic testing including: referral to specialist genetic services; informal peer consultation; and/or formal case review at multidisciplinary (MDT) meetings. New models of practice are needed due to the complexity and volume of tests and the available clinical genetics workforce. Participants identified that medical specialists and other clinical HPs may require additional education/training in genomics. Additional to university and College-based training, hands-on workshops (CME) with supporting online learning to cover basics of genomics was a preferred approach to education delivery. Interestingly, experiential learning activities such as MDT meetings, department meetings, 'hallway conversations', scientific conferences and involvement in research were also identified. However, these were not perceived as educational opportunities per se and pose challenges for evaluation, such as benchmarking against competencies. Models of practice in genomic medicine and HPs' roles are evolving. Education/training, at a number of levels, is essential to facilitate best practice to ensure successful implementation of genomics in healthcare.

B. McClaren: None. Z. Prichard: None. A.E. Nisselle: None. E. Crellin: None. M. Janinski: None. L. Ng: None. E. Zilliacus: None. K. Dunlop: None. D. Graves: None. B. Terrill: A. Employment (full or part-time); Modest; Genome.One. A. Newson: None. C. Gaff: None. S.A. Metcalfe: None.

\section{EPL3.5}

Exploring the experiences of genetic health professionals with adoptees

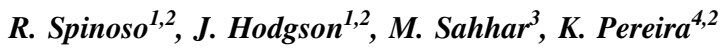 \\ ${ }^{1}$ University of Melbourne, Melbourne, Australia, ${ }^{2}$ Murdoch \\ Children's Research Institute, Melbourne, Australia, ${ }^{3}$ Victorian \\ Clinical Genetic Services, Melbourne, Australia, ${ }^{4}$ Genetic \\ Support Network of Victoria, Melbourne, Australia
}

Background: Family history is important in determining an individual's risks of genetic conditions. Adoptees often have limited knowledge of their family history. The clinical and psychosocial implications of this, as well as the genetic counselling strategies employed by professionals to support these clients have not been previously explored.

Aim: This research aimed to explore the experiences of genetic health professionals with adopted clients. In particular, it sought to identify the issues posed and the counselling strategies employed when working with adoptees.

Methods: This study took a mixed-methods approach comprising quantitative and qualitative data collection. A survey was conducted of members of the Australasian Society of Genetic Counsellors (ASGC) and Australasian Association of Clinical Geneticists (AACG) and statistical analysis was performed. The survey data informed the qualitative phase, where professionals, who have worked with an adoptee, were recruited for semi-structured 
interviews. Interviews were audio-taped, transcribed verbatim, coded and analysed thematically to identify common themes.

Results: A total of 68 survey responses were received and eight interviews were conducted. A number of practical challenges as well as counselling issues specific to the adoptees limited knowledge of family history were identified. Lastly, interviewees made a number of recommendations to assist genetic health professionals when working with these clients.

Conclusions: The findings from this study will help inform genetic counselling practice when working adoptees by raising awareness of the challenges posed by limited family history knowledge and providing insight into the strategies and interventions employed by professionals when working with these clients.

R. Spinoso: None. J. Hodgson: None. M. Sahhar: None. K. Pereira: None.

\section{EPL3.6}

Learning the role of genomics in human health: the serious games experience

S. Oliveri ${ }^{1}$, R. Mainetti ${ }^{2}$, I. Cutica ${ }^{1}$, A. Gorini ${ }^{1}$, N. A. Borghese ${ }^{2}$, G. Pravettoni ${ }^{1}$

${ }^{1}$ Department of Oncology and Hemato-Oncology, Università degli Studi di Milano, Milan, Italy, ${ }^{2}$ Applied Intelligent Systems Laboratory AISLab, Dept. of Computer Science, Università degli studi di Milano, Milan, Italy

Introduction: Despite the growth of genomics in health care, current approaches to genetic and genomic education do not adequately prepare the public to understand personal health issues related to genomic medicine. Serious games can play an important role in bringing genomic science closer to the public, as they supplement traditional education through interactive modalities.

Materials and Methods: Starting from the social cognitive theory, which recognizes the importance of selfefficacy as a prerequisite to manage health information, and web-based learning environments theories, we designed two mini-games focused on heredity and mutation and an adventure game, with an avatar storytelling, focused on genetic testing, inheritance, and genes-behaviorenvironment interaction. The playability testing was conducted with a sample of 30 participants, subdivided into 3 equal groups and randomly assigned to each game. Participants were asked to report what they learnt by playing the games and any negative issue they found during the play. They also completed the Games Experience Questionnaire.
Results: Overall participants figured out games contents and experienced moderate levels of immersion and flow, suggesting a balance between fun and cognitive engagement. Players experienced low levels of negative feelings such as annoyance and frustration and prevalence of positive emotions. However, they reported that the games should be more intuitive.

Conclusions: Playability results showed that the serious games developed might efficiently improve public's knowledge of basic biology and genetic concepts, and foster public's ability in using genomic information for healthrelated decision making. The Swedish Foundation for Humanities and Social Sciences. Grant nr. M13-0260:1

S. Oliveri: None. R. Mainetti: None. I. Cutica: None. A. Gorini: None. N.A. Borghese: None. G. Pravettoni: None.

\section{EPL4 What's New in Hereditary Cancer}

\section{EPL4.1}

I remember the feeling not the gene: Families' experiences of and attitudes towards genetic testing in childhood cancer

\author{
B. C. McGill ${ }^{1,2}$, C. E. Wakefield ${ }^{1,2}$, J. Vetsch ${ }^{1,2}$, R. J. Cohn ${ }^{1,2}$, \\ M. Warby ${ }^{3,4}$, K. M. Tucker ${ }^{3,4}$
}

${ }^{1}$ School of Women's and Children's Health, UNSW Sydney, Sydney, Australia, ${ }^{2}$ Kids Cancer Centre, Sydney Children's Hospital, Randwick, Australia, ${ }^{3}$ Hereditary Cancer Clinic, Department of Medical Oncology, Prince of Wales Hospital, Randwick, Australia, ${ }^{4}$ Prince of Wales Clinical School, UNSW Sydney, Sydney, Australia

Introduction: Genetic technologies have great potential in the paediatric setting in diagnosing hereditary cancer syndromes and predicting cancer risk in children. However, we know little about families' experiences of the counselling and testing process. We aimed to synthesise the existing evidence on children and young adults' understanding of genetic testing for disease predisposition, followed by qualitative analysis of the understanding, experiences, and attitudes of families affected by hereditary childhood cancer/tumour syndromes.

Methods: Study one: We conducted a systematic literature review to examine children and young adults' ( $\leq 21$ years-old) understanding of, and attitudes towards, genetic testing for disease predisposition across illnesses. Study two: We conducted semi-structured interviews with parents and young adults $(<16$ years-old at the time of referral) who were offered cancer predisposition genetic testing. 
Results: Study one: We identified 20 studies representing the views of 1813 children and young adults (1498 population-risk, 315 affected/at-risk). Children and young adults generally hold positive attitudes towards clinical genetic testing, but misunderstandings were evident in terms of the implications of a genetic diagnosis. Study two: We interviewed 35 participants from 28 families $\left(M_{\text {age }}\right.$ at diagnosis $=10.3$ years, $S D=5.5$ ). Families expressed high satisfaction with genetic services and reported benefits to receiving a genetic diagnosis. However many families reported unmet genetic information needs.

Conclusions: Families have largely positive experiences of genetic testing. However, unmet information needs were common. For the effective utilisation of genetic healthcare information in the paediatric cancer setting this barrier needs to be addressed through the provision of developmentally-appropriate educational resources.

B.C. McGill: None. C.E. Wakefield: None. J. Vetsch: None. R.J. Cohn: None. M. Warby: None. K.M. Tucker: None.

\section{EPL4.2}

Companions or patients? The impact of family presence in genetic counseling for hereditary breast cancer

\section{S. Barnoy ${ }^{1}$, R. Gilbar $^{2,3}$ \\ ${ }^{1}$ Tel-Aviv University, Tel Aviv, Israel, ${ }^{2}$ Netanya Academic College, Netanya, Israel, ${ }^{3}$ University of Leicester, Leicester, United Kingdom}

Introduction: As other areas of medical practice, relatives accompany patients to genetic consultations. However, unlike other areas, the consultations may be relevant to the relatives' health because they may be at risk of developing the same genetic condition as the patient. The presence of relatives in genetic consultation may affect the decisionmaking process and it raises questions about the perception of patient autonomy and the way it is practiced in genetics. However, these issues have not been examined in previous empirical studies.

Methods: This was a qualitative study using semistructured, face-to-face interviews with 28 clinicians working in the area of inherited breast cancer. The study was based on a framework analysis approach.

Results: The findings indicate that family presence has an impact on the patient's decisions to undergo genetic testing and preventative operations when she is diagnosed as a carrier. The findings further indicate that unlike other areas of medical practice, blood relatives who are present in consultations are perceived by clinicians as patients or potential patients, and this in turn increases their involvement in discussions in the consultation room. Finally, the findings indicate that decisions are made in a social context, where the relatives' views are heard and taken into account.

Conclusions: The findings suggest that the conventional bioethical approach to autonomy, which perceives the decision-making unit comprised of a clinician and an individual patient, is challenged in genetics. The findings thus require bioethicists, lawyers and policy-makers to consider whether this individualistic approach is still valid and applicable.

S. Barnoy: None. R. Gilbar: None.

\section{EPL4.3}

Uptake of polygenic risk information among women at potentially high breast cancer risk

T. Yanes ${ }^{1}$, B. Meiser ${ }^{1}$, R. Kaur ${ }^{1}$, M. Scheepers-Joynt ${ }^{2}$, M. Young ${ }^{3,4}$, K. Barlow-Stewart ${ }^{5}$, T. John ${ }^{6}$, M. Harris ${ }^{7}$, Y. Antill ${ }^{8}$, J. Halliday ${ }^{9,10}$, J. Burke ${ }^{11}$, T. Roscioli ${ }^{12}$, P. Mitchell ${ }^{1}$, P. James ${ }^{2}$

${ }^{1}$ University of New South Wales, Sydney, Australia, ${ }^{2}$ Parkville Familial Cancer Centre, Peter MacCallum Cancer Centre, Melbourne, Australia, ${ }^{3}$ Garvan Institute of Medical Research, Sydney, Australia, ${ }^{4}$ Parkville Familial Cancer Centre, Peter MacCallum Cancer Centre, Melbourne, Australia, Melbourne, Australia, ${ }^{5}$ University of Sydney, Sydney, Australia, ${ }^{6}$ Clinical Genetics Service, Austin Health, Melbourne, Australia,

${ }^{7}$ Familial Cancer Clinic, Monash Medical Centre, Melbourne, Australia, ${ }^{8}$ Familial Cancer Clinic, Cabrini Hospital, Melbourne, Australia, ${ }^{9}$ Murdoch Children's Research Institute, Melbourne, Australia, ${ }^{10}$ University of Melbourne,, Melbourne, Australia, ${ }^{11}$ Tasmanian Clinical Genetics Service, Hobart, Australia, ${ }^{12}$ Department of Medical Genetics, Sydney Children's Hospital, Sydney, Australia

Background: Despite increasing scientific evidence regarding the utility of polygenic risk score (PRS) for families at high-risk of breast cancer, research findings are yet to be integrated into clinical practice. Before integrating polygenic information into clinical practice it is important to understand the psychological implications. This prospective study aims to assess uptake of breast cancer polygenic risk information, and ascertain the psychosocial and behavioural implications of receiving this information.

Methods: Eligible women are invited to participate and receive their breast cancer PRS. Eligibility: affected and unaffected women currently enrolled in the Variants in Practice Study, who have a high or low PRS, and a personal and/or family history of breast cancer where genetic testing for $B R C A 1 / 2$ is negative. Participants complete three selfadministered questionnaires: T1 prior to result, T2 two weeks and T3 one year post receipt of PRS. 
Results: As of January 2018, 132/153 (86\%) participants reported interest in receiving their PRS with 21/153 (14\%) declining. Primary reasons cited to receive PRS included: helping research (84\%) and family members $(68 \%)$, and to manage breast cancer risk (64\%). Preliminary analysis showed uptake of PRS is associated with having daughters $(\mathrm{r}=0.16, \mathrm{p}=0.041)$, higher uncertainty avoidance $(\mathrm{r}=$ $0.38, \mathrm{p}=<0.001)$ and higher perceived severity of breast cancer $(r=0.20, p=0.018)$. Conclusion: There is strong interest in receiving PRS among women at high-risk of breast cancer. Recruitment is ongoing, with additional data regarding short-term and long-term psychological and behavioural impact of receiving PRS to be collected.

T. Yanes: None. B. Meiser: B. Research Grant (principal investigator, collaborator or consultant and pending grants as well as grants already received); Modest; AstraZeneca. R. Kaur: None. M. Scheepers-Joynt: None. M. Young: None. K. Barlow-Stewart: None. T. John: None. M. Harris: None. Y. Antill: None. J. Halliday: None. J. Burke: None. T. Roscioli: None. P. Mitchell: None. P. James: None.

\section{EPL4.4}

Uncertainty related to multigene panel testing for cancer: a qualitative study on counsellors' and counselees' views

N. M. Medendorp ${ }^{1}$, M. A. Hillen ${ }^{1}$, L. Murugesu ${ }^{1}$, C. M. Aalfs ${ }^{1}$, A. M. Stiggelbout ${ }^{2}$, E. M. A. Smets ${ }^{1}$

${ }^{1}$ Academic Medical Center, Amsterdam, Netherlands, ${ }^{2}$ Leiden University Medical Center, Leiden, Netherlands

Introduction: Multigene panel testing for cancer improves the identification of genetic causes in families with possible cancer syndromes. Panel tests may however yield uncertainty, for example when variants of unknown significance are identified. Since insight is limited into how these uncertainties play a role in counseling, we explored counsellors' and counselees' experiences with uncertainty, and how they discuss uncertainties and decide about multigene panel testing.

Materials and Methods: Six focus groups were conducted including a total of 38 counsellors. Twelve counselees who had received genetic counselling about a multigene panel test were interviewed individually. The transcribed audio recordings were analyzed inductively by two coders and data were collated to distill broader themes.

Results: Counsellors mostly experienced difficulty in deciding what uncertain information to communicate to counselees and how to do so. The extent and manner of providing uncertain information differed between centers and between counsellors. Counsellors attached more value to counselees' preferences in decision making compared to targeted DNA tests, because uncertainty is increased. Counselees experienced difficulty in recalling which uncertainties related to multigene panel testing had been discussed during genetic counselling. They primarily reported to have experienced uncertainty about their own and their relatives' risk of developing cancer. Counselees felt they had had a say in the decision.

Conclusions: Counsellors need more guidance on whether and how to convey uncertainty to counselees. Undesirable practice variation in the communication of uncertainty may be prevented by determining what information should minimally be discussed to enable informed decision making.

N.M. Medendorp: None. M.A. Hillen: None. L. Murugesu: None. C.M. Aalfs: None. A.M. Stiggelbout: None. E.M.A. Smets: None.

\section{EPL4.5}

The efficacy of genetic counselling for familial colorectal cancer: a meta-analysis

A. Ciucă $\breve{l}^{1}$ R. Moldovan ${ }^{1}$, S. Pintea ${ }^{1}$, A. Băban ${ }^{1}$, T. Clancy $^{2}$

${ }^{1}$ Department of Psychology, Babe-Bolyai University, ClujNapoca, Romania, ${ }^{2}$ Genomic Medicine, St Mary's Hospital, Manchester, United Kingdom

Introduction: Prospective studies and RCTs have shown that genetic counselling is effective for a number of conditions in relation to outcomes such as risk perception and knowledge. By statistically combining data from individual studies, a meta-analysis can provide a precise estimate of an intervention overall effect. This approach allows us to assess the efficacy of genetic counselling for various conditions and outcomes. The aim of the current quantitative meta-analysis was to analyse the efficacy of genetic counselling for familial colorectal cancer and explore characteristics that might influence the direction or magnitude of the relation between the intervention and the outcome.

Method: An extensive electronic search was conducted investigating the literature published until January 2018. This identified 2121 articles, 30 of which met the inclusion criteria. Effect size parameters and sample sizes for all variables in each study were included.

Results: Data showed that genetic counselling has an overall statistically significant effect size, of small magnitude. Results indicate that genetic counselling is effective for affective, cognitive and behavioural outcomes; for individuals with both personal and family history; whether they pursue testing or not; regardless of the purpose of testing; and, with the exception of uninformative results, regardless of the mutation status. Analyses also show that 
teams that included a genetic counsellor generated a significantly larger effect.

Conclusion: We found that genetic counselling is beneficial at both post- intervention and follow-up, which indicates that the benefits gained during the genetic counselling session are maintained over time.

A. Ciucă: None. R. Moldovan: None. S. Pintea: None. A. Băban: None. T. Clancy: None.

\section{EPL4.6}

Moving into the mainstream: Treatment focussed genetic testing a screening tool or diagnostic resource?

N. Hallowell ${ }^{1}$, S. Wright ${ }^{2}$, D. Stirling ${ }^{3}$, J. Lawton $^{3}$, C. Gourlay ${ }^{3}$, O. Young, ${ }^{4}$ M. Porteous ${ }^{3}$

${ }^{1}$ University of Oxford, Oxford, United Kingdom, ${ }^{2}$ University of Edinburgh, edinburgh, United Kingdom, ${ }^{3}$ University of Edinburgh, Edinburgh, United Kingdom, ${ }^{4}$ Western General Hospital, Edinburgh, United Kingdom

For many years clinical geneticists used $B R C A 1$ and 2 genetic testing as a screening test to identify those who had a genetic predisposition to develop breast and ovarian cancer. More recently $B R C A$ testing is used to direct the treatment of patients with breast or ovarian cancer, either informing their adjuvant chemotherapy regimes or surgical management. Thus, the role of BRCA testing in patient management has changed from that of a screening tool, used by a small group of specialists, to a diagnostic/treatment resource employed in mainstream cancer care (oncology and breast surgery). Using interview data, this paper will explore how healthcare professionals $(n=21)-$ oncologists, breast surgeons and clinical genetics professionals in one UK teaching hospital - conceive of this new application of $B R C A$ testing. We will show that healthcare professionals prioritise treatment focussed $B R C A$ testing (TFGT) very differently from the way it is intended within the patient pathway. We will argue the introduction of $B R C A$ testing in the mainstream raises issues for professional identity and that in describing their engagement with $B R C A$ testing healthcare professionals involved in the treatment focussed testing pathway engage in professional boundary maintenance, as demonstrated by their reconfiguration of the purpose of BRCA testing in this context. This paper, will thus, enable us to interrogate the screeningdiagnosis dichotomy and provide insight into the issues that will require addressing prior to more widespread implementation of genomics in the NHS. Breast Cancer Now Grant no: [2016MayPR700].

N. Hallowell: None. S. Wright: None. D. Stirling: None. J. Lawton: None. C. Gourlay: None. O. Young: None. M. Porteous: None.
EPL5 To know or not to know

\section{EPL5.1}

1 in 39 individuals carries a dominant high-risk disease allele

H. G. Yntema ${ }^{1}$, L. Haer-Wigman ${ }^{1}$, A. Vulto-van Silfhout ${ }^{1}$, V. van der Schoot $^{2}$, C. Gilissen ${ }^{1}$, H. G. Brunner ${ }^{1,3}$, I. Feenstra ${ }^{1}$, L. E. L. M. Vissers ${ }^{1}$

${ }^{1}$ Dept Human Genetics, Nijmegen, Netherlands, ${ }^{2}$ Dept Clinical Genetics, Maastricht, Netherlands, ${ }^{3}$ Department of Clinical Genetics, Maastricht, Netherlands

Clinical genomic sequencing can identify pathogenic variants unrelated to the initial clinical question, but of medical relevance to the patient and their families. To promote standardized reporting of these incidental (unintentionally detected in analysis) and/or secondary findings (deliberate analysis of available data), the American College of Medical Genetics and Genomics (ACMG) published 59 medically actionable genes recommended for return of such findings. With ongoing discussions on obligatory genetic testing of employees and disclosure of the results to their employers, it is of crucial importance to obtain unbiased insight on the potential risks and benefits. We analysed WES data of 1,640 anonymized healthy Dutch individuals to establish the frequency of medically actionable disease alleles in the general population. Our study shows that when healthy individuals would be routinely screened for mutations in the 59 ACMG medically actionable genes, at least 1 in 39 individuals $(2.6 \%)$ has a lifetime increased risk for a severe dominant disease. Additionally, we identified 36 individuals $(2.2 \%)$ to be a carrier of a recessive pathogenic disease allele. Whereas it is recommended not to return carrier status to these individuals unless identified as biallelic mutations, one may wonder whether carrier status is not relevant for reproductive decisions, especially if more prevalent diseases, such as cystic fibrosis, were to be included in this communication. Hence, our results contribute significantly to the debate on genetic risk factor screening in healthy individuals and the discussion if the benefits of this knowledge outweigh the risk of living with uncertainty and possible stigmatization.

H.G. Yntema: None. L. Haer-Wigman: None. A. Vulto-van Silfhout: None. V. van der Schoot: None. C. Gilissen: None. H.G. Brunner: None. I. Feenstra: None. L.E.L.M. Vissers: None.

\section{EPL5.2}

To report or not to report? That's not the only question! Analysis of VUS reporting, variant reinterpretation, and recontact policies in clinical genomics consent forms 


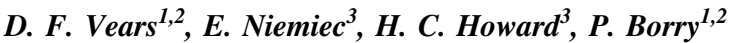

${ }^{1}$ Centre for Biomedical Ethics and Law, Department of Public Health and Primary Care, KU Leuven, Leuven, Belgium, ${ }^{2}$ Leuven Institute for Human Genomics and Society, Leuven, Belgium, ${ }^{3}$ Centre for Research Ethics and Bioethics, Uppsala University, Uppsala, Sweden

Whether laboratories and clinics should report variants of uncertain significance (VUS) to clinicians and/or patients, reinterpret VUS in response to the growing knowledge in the field, reissue reports to clinicians based on any revised classifications, and recontact patients, are all key unsolved issues in the clinical use of next-generation sequencing (NGS). We systematically analyzed 58 consent forms in English being used in the USA, Europe, and other countries, to investigate their policies for a) reporting VUS, b) reinterpreting variants, including who should initiate this, and c) recontacting patients and the mechanisms for undertaking any recontact. One-third (20/58) of the forms did not mention VUS in any way. Of the 38 forms that did, only half provided some description or definition for what a VUS is. Nineteen forms explicitly stated that reinterpretation of variants for clinical purposes may occur. An additional 12 forms did not explicitly state that they report VUS but reporting was implied. Twenty forms referred to the idea that reinterpretation of variants for clinical purposes may take place, eight mentioned that variants might be reclassified, and five stated more information might come to light in the future. Less than half mention recontact for clinical purposes, varying as to whether the laboratory, patient or clinician should initiate this. The variability in variant reporting, reinterpretation, and recontact policies and practices revealed by our analysis may lead to diffused responsibility and result in missed opportunities for patients or family members to receive a diagnosis in response to updates in variant classification.

D.F. Vears: None. E. Niemiec: None. H.C. Howard: None. P. Borry: None.

\section{EPL5.3}

The patient voice in design of systems to share clinical genomic sequencing data

M. Martyn ${ }^{1,2,3}$, P. Gleeson ${ }^{4}$, C. Walker ${ }^{1,3,5}$, H. Chia ${ }^{4}$, A. KangaParabia ${ }^{1,3}$, Melbourne Genomics Health Alliance, C. L. Gaff (1,3 $^{1,3}$

${ }^{1}$ Melbourne Genomics Health Alliance, Parkville, VIC, Australia, ${ }^{2}$ Murdoch Children's Research Institute, Parkville, VIC, Australia, ${ }^{3}$ The University of Melbourne, Parkville, VIC, Australia, ${ }^{4}$ Melbourne Law School, Parkville, VIC, Australia, ${ }^{5}$ Chronic Illness Alliance, Melbourne, VIC, Australia
Genomic data from clinical testing is potentially valuable for research. In contrast to community and research participant views, patient expectations about the research use of germline data from clinical genomic testing is unknown.

We have addressed this gap by surveying patients receiving genomic testing through clinical care and consenting, or not, to use of their data for research.

Surveys include categorical and open-ended questions on how clinical genomic data should be stored, accessed and used for research. Categorical data is analysed using descriptive statistics, and open-ended questions using qualitative content analysis. The survey response rate is $70 \%(566 / 805)$.

To date, $99 \%(857 / 868)$ of patients agree to additional research use of their clinical genomic data. Despite the clinical community's concerns about effectiveness of pretest counselling, 96\% (511/531) of respondents indicate they had enough information before making their decision.

Patient expectations about control over the use of their data is informing the design of systems around data use. Blanket consent and refusal were rarely preferred, but there was no clear preference between opt-in or opt-out consent for each study, nor for reinitiating contact to withdraw.

Patients were surprisingly supportive of data sharing, indicating they would allow a range of organisations to access data and even re-identify them. A minority (5\%, 21/ 470) felt it would be easy for their data to be reidentified and would be concerned if this occurred.

Patient voices are informing data governance, design and prioritisation of a single system for research access to statewide clinical genomic sequencing data.

M. Martyn: None. P. Gleeson: None. C. Walker: None. H. Chia: None. A. Kanga-Parabia: None. C.L. Gaff: None.

\section{EPL5.4}

Predicting willingness to receive four different types of genetic risk information - A population based study

\section{A. Haukkala ${ }^{1}$, M. Vornanen ${ }^{1}$, O. Halmesvaara ${ }^{1}$, H. Konttinen ${ }^{1}$,} H. Kääriäinen ${ }^{2}$, K. Aktan-Collan ${ }^{1}$

${ }^{1}$ University of Helsinki, Helsinki, Finland, ${ }^{2}$ National Institute for Health and Welfare, Helsinki, Finland

Introduction: in general, people wish to know about their health-related genetic risk information, but do not necessarily differentiate between different types of risk information. We created a three-minute video about four categories of genetic risk information and examined predictors of individuals' intentions to receive each category of risk information. 
Material and Methods: 792 respondents from a random population sample of 18 to 64 year olds participated in a web survey, which included the three -minute video. The video covered four categories of genetic risk information (actionable, non-actionable, multifactorial, carrier status). We measured participants' attitudes, perceived norms and intention toward learning about each type of risk information. Education, gender, anxiety, genetic knowledge and need for cognitive closure were also investigated as potential predictors of intentions.

Results: Respondents evaluated the presented video as useful and comprehensible. Respondents were willing to learn their actionable, multifactorial and carrier risk information, but were less interested in non-actionable heritable risk information. Need for cognitive closure was related to willingness to learn actionable $(\beta=0.08$, $\mathrm{p}=.030)$, multifactorial $(\beta=0.09, \mathrm{p}=.013)$, and carrier status risk information $(\beta=0.11, p=.002)$, while other predictors were non-significant. More educated participants were less motivated to learn their nonactionable risks $(\beta=-0.09, \mathrm{p}=.017)$.

Conclusion: Respondents had a positive attitude toward genetic testing and were interested to learn different types of genetic risks. Need for cognitive closure had moderate association to intentions while many other predictors, including genetic knowledge, were not related to intentions.

A. Haukkala: None. M. Vornanen: None. O. Halmesvaara: None. H. Konttinen: None. H. Kääriäinen: None. K. Aktan-Collan: None.

\section{EPL5.5}

Disclosure of incidental findings (IFs) and variants of uncertain significance (VUS) to patients: what happens in practice?

\section{J. el Mecky ${ }^{1}$, M. Plantinga ${ }^{1}$, A. Fenwick ${ }^{2}$, A. Lucassen ${ }^{2}$, I. van} Langen ${ }^{1}$

${ }^{1}$ University Medical Centre Groningen, Groningen, Netherlands, ${ }^{2}$ University of Southampton, Southampton, United Kingdom

Introduction: It is well-known that the introduction of whole genome sequencing approaches increases the potential for IFs and VUS. A host of guidelines have been published on clinical management of IFs and, to a lesser degree, of VUS. But what actually happens in practice? Currently, very little has been published on the clinical decisionmaking process regarding which IFs and VUS should be disclosed to patients (and which should not) and how this differs among different healthcare professionals and clinics.

Methods: This study involves observation of one genetic centre's clinical multidisciplinary team meetings over a period of 1.5 years, as well as semi-structured interviews with healthcare professionals (HCPs) across a wide range of clinical genetics settings in all (8) Dutch genomics centres. It explores how healthcare professionals make decisions regarding whether and which genetic IFs and VUS to communicate to patients: what factors play a role in this process and how are benefits and disadvantages of disclosing these results weighed up? How do healthcare professionals view the concept of patient autonomy and consent in relation to genetic findings? This study attempts to unravel the complexities behind clinical management of IFs and VUS and provide new insights for policy.

Results: Initial results show important differences in approaches to IFs and VUS across different clinics and diagnostic settings. For example, some centres offer patients a choice which IFs (including non-actionable IFs) they would like to receive, whereas in others, HCPs feel it is the professional's responsibility to make that choice.

J. el Mecky: None. M. Plantinga: None. A. Fenwick: None. A. Lucassen: None. I. van Langen: None.

\section{EPL5.6}

Consent for Genetic Testing and Disclosure of Results: Shifting the Paradigm to Non-Genetics Clinicians

K. E. Ormond ${ }^{1}$, M. L. Hallquist ${ }^{2}$, E. Tricou ${ }^{2}$, K. Brothers ${ }^{3}$, C. R. Coughlin II ${ }^{4}$, L. Hercher ${ }^{5}$, L. Hudgins ${ }^{1}$, H. Levy ${ }^{6}$, H. Peay ${ }^{7}$, M. Roche ${ }^{8}$, M. Stosic ${ }^{9}$, M. Smith ${ }^{10}$, W. Uhlmann ${ }^{11}$, K. Wain ${ }^{12}$, W. A. Faucett ${ }^{12}$, A. H. Buchanan ${ }^{12}$

${ }^{1}$ Stanford University, Stanford, CA, United States, ${ }^{2}$ Geisinger, Danville, PA, United States, ${ }^{3}$ University of Louisville, Louisville, KY, United States, ${ }^{4}$ University of Colorado, Denver, CO, United States, ${ }^{5}$ Sarah Lawrence University, Bronxville,

NY, United States, ${ }^{6}$ Johns Hopkins University, Baltimore, MD, United States, ${ }^{7} R T I$ International, Research Triangle, $N C$, United States, ${ }^{8}$ University of North Carolina, Chapel Hill, NC, United States, ${ }^{9}$ Natera, Inc, San Carlos, CA, United States,

${ }^{10}$ Northwestern University, Chicago, IL, United States,

${ }^{11}$ University of Michigan, Ann Arbor, MI, United States,

${ }^{12}$ Geinsinger, Danville, PA, United States

Timely access to genetic testing and counseling remains an issue that will only grow as genetics assumes a larger role in routine healthcare; it is unclear how best to increase access while providing patients with adequate genetic testing education and support. ClinGen's Consent and Disclosure Recommendations working group (CADRe) has developed and evaluated rubrics for determining a suggested communication approach for consent and disclosure. We propose three possible communication approaches: (1) traditional genetic counseling (TGC) with a genetics specialist, (2) targeted discussion with an ordering clinician, or 
(3) brief communication supported by educational resources. The CADRe recommendations provide guidance regarding which conditions and indications would benefit most from TGC where detailed discussion, complicated test selection, and psychosocial support are provided, with the goal of directing genomics expertise to those patients for whom it is most impactful. We review the application of the model using the ACMG Secondary Findings v2.0 gene list, examining each in the context of specific indications for genetic testing, including: confirmation of a clinical diagnosis, testing an individual with a suggestive personal history, testing an unaffected individual with a suggestive family history, and testing an unaffected individual for a known familial variant. Our current classification trends suggest that much of the counseling associated with genetic testing can be triaged and transitioned to targeted discussions with ordering physicians. We support shifting the paradigm of genetic testing to emphasize the use of genetics providers in complex cases that require specialized genetics expertise.

K.E. Ormond: None. M.L. Hallquist: None. E. Tricou: None. K. Brothers: None. C.R. Coughlin II: None. L. Hercher: None. L. Hudgins: None. H. Levy: None. H. Peay: None. M. Roche: None. M. Stosic: A. Employment (full or part-time); Modest; Natera Inc. M. Smith: None. W. Uhlmann: None. K. Wain: None. W.A. Faucett: None. A.H. Buchanan: None.

\section{EPL6 Perinatal decision-making}

\section{EPL6.1}

Stakeholder views towards prenatal and postnatal fetal mesenchymal stem cell infusions for osteogenesis imperfecta

\section{Hill ${ }^{1,2}$, C. Lewis ${ }^{1,2}$, M. Riddington ${ }^{3}$, B. Crowe ${ }^{3}$, C. DeVile ${ }^{3}$,}

C. Götherström ${ }^{4}$, L. S. Chitty ${ }^{1,2}$

${ }^{1}$ NE Thames Regional Genetics Service, Great Ormond Street Hospital NHS Foundation Trust, London, United Kingdom,

${ }^{2}$ Genetics and Genomic Medicine, UCL Great Ormond Street Institute of Child Health, London, United Kingdom,

${ }^{3}$ Osteogenesis Imperfecta Service, Great Ormond Street

Hospital for Children NHS Foundation Trust, London, United

Kingdom, ${ }^{4}$ Center for Hematology and Regenerative Medicine,

Karolinska Institutet, Stockholm, Sweden

Introduction: The Boost Brittle Bones Before Birth (BOOSTB4) clinical trial is investigating the safety and efficacy of using prenatal and early postnatal fetal mesenchymal stem cell infusion for severe osteogenesis imperfecta (OI). We are exploring stakeholder views to understand perceived benefits or concerns, identify ethical issues and establish support and counselling needs.

Materials and Methods: Semi-structured qualitative interviews were conducted with four groups; 1 . Parents / carers of children affected with OI; 2 . Adults affected with OI; 3. Health professionals; 4. Patient advocates. Interviews were digitally recorded, transcribed verbatim and analysed using thematic analysis.

Results: Interviews with 50 participants revealed generally positive views towards stem cell infusions for OI. Early treatment was considered advantageous for reducing severity by avoiding fractures at a time of rapid bone development and could bring psychological benefits for parents, as it offers hope. Common concerns were procedure safety, short/long-term side effects, and whether infusions would be effective. Difficulties inherent in decision-making were frequently discussed, as treatment efficacy is unknown and, by necessity, parents will make decisions at a time of vulnerability and are potentially willing to "do anything" to help their child. Support needs may differ where there is a family history of OI compared to an unexpected diagnosis of OI.

Conclusions: Good communication, significant support and time for reflection during the decision-making process will be crucial to allow parents to make informed decisions about stem cell infusions in the management of OI.

European Union Horizon 2020 research and innovation programme: grant agreement No 681045 .

M. Hill: None. C. Lewis: None. M. Riddington: None. B. Crowe: None. C. DeVile: None. C. Götherström: None. L.S. Chitty: None.

\section{EPL6.2}

Factors contributing to new parents' perspectives on retention and secondary use of neonatal dried bloodspots A mixed methods study in the Netherlands

M. E. Jansen ${ }^{1,2}$, L. J. M. van den Bosch ${ }^{3}$, M. J. Hendriks ${ }^{1}$, M. M. J. Scheffer ${ }^{1}$, M. L. Heijnen ${ }^{3}$, C. M. W. Douglas ${ }^{4}$ C. G. van $\mathrm{El}^{1}$

${ }^{1} A P H$ research institute VU University Medical Centre, Amsterdam, Netherlands, ${ }^{2}$ Centre for Health Protection, National Institute for Public Health and the Environment, Bilthoven, Netherlands, ${ }^{3}$ Centre for Population Screening, National Institute for Public Health and the Environment, Bilthoven, Netherlands, ${ }^{4}$ Department of Technology and Society Studies, Maastricht University, Maastricht, Netherlands

Introduction: Neonatal bloodspot screening (NBS) identifies conditions to minimize irreversible damage. NBS policies guide a comprehensive system including processes for storage of neonatal dried blood spots (NDBS). NDBS 
policies have been subject of public debates internationally suggesting that the public's perceptions of NDBS policy are not always on par with policies in place. The current study aims to provide insight in relevant factors for new parents in the Netherlands regarding retention and secondary use. These factors can be taken into account when developing or updating NDBS policies.

Methods A mixed methods design was used combining an online survey $(n=765)$, focus group interviews $(n=6$, 37 participants), and individual in-depth interviews $(\mathrm{n}=7)$. The discussed topics included: parental information; obtaining informed consent; support for retention; and support for secondary use. The study population consisted of Dutch-speaking new parents: pregnant women ( $\geq 20$ weeks) and/or their partner, and parents of at least one child ( $\leq 5$ years).

Results Overall, participants were positive about prolonged retention and different types of secondary use. The participants indicated transparency and safety in NDBS policy and practice as important overarching themes. Transparency was discussed in subthemes such as knowledge and engagement, while safety translated into subthemes such as autonomy, anonymity, and trust.

Conclusions Needs were expressed for easily accessible information, adequate communication on the retention and (potential) use of NDBS, clearly described safeguards for privacy protection, a more active consent process, retrievable information and regulation for the actors conducting NDBS research, and participation of new parents in decisions on secondary use.

M.E. Jansen: None. L.J.M. van den Bosch: None. M.J. Hendriks: None. M.M.J. Scheffer: None. M.L. Heijnen: None. C.M.W. Douglas: None. C.G. van El: None.

\section{EPL6.3}

\section{Slippery slope for oocyte donations}

\section{P. Tulay ${ }^{1}$, O. Atilan ${ }^{2}$}

${ }^{1}$ Near East University, Nicosia, Cyprus, ${ }^{2}$ Nicosia IVF Clinic, Nicosia, Cyprus

Introduction: In recent years oocyte donation programs have gained more attention. Although in some countries the donation programs are tightly regulated; in some, there are no strict guidelines or regulations. This introduces many issues in such countries where the oocyte donations become the drive of health tourism. The aim of this study was to evaluate the ethical aspects of oocyte donations and donors' awareness of the consequences in undergoing donation programs.

Materials and Methods: A detailed questionnaire based survey was conducted with the donors to evaluate the main reason of the involvement in the donation program, donor's knowledge and awareness of risk factors. Since in the recent years, genetic screening has become more common, the donors were questioned about their knowledge on the subject.

Results: Majority of the donors (70\%) were undergoing donation programs due to financial problems. The donors were especially not aware that DNA from their blood samples could be tested for genetic variations or predispositions. Furthermore, they did not know about genetic anonymity and the possibility of being exposed later on in life.

Conclusion: The health professionals have the main duty to inform the donors about any possible problems they may face in the donation programs. Payment to oocyte donors is a slippery slope in oocyte donation programs. High compensation may make women to think that donation is a profession without considering possible risks. Furthermore, with the wider use of direct-to-consumer genetic testing, genetic anonymity may be at risk, thus the donors have to be counselled properly.

P. Tulay: None. O. Atilan: None.

\section{EPL6.4}

Development and pilot study of the prenatal informed decision-making (PRENID)-scale: a measure for informed decision-making in first trimester prenatal screening

\section{M. Bakkeren', M. G. Polak ${ }^{2}$, F. K. C. Kisters ${ }^{2}$, R. H. Galjaard $^{1}$, A. Tibben ${ }^{3}$, S. R. Riedijk ${ }^{1}$}

${ }^{1}$ Erasmus Medical Centre, Rotterdam, Netherlands, ${ }^{2}$ Erasmus University Rotterdam, Rotterdam, Netherlands, ${ }^{3}$ Leiden

University Medical Center, Leiden, Netherlands

Introduction: Pregnant women in the Netherlands can choose between NIPT, the combined test (CT) and not participating in first trimester screening (FTS). It is unclear whether pregnant women are making informed decisions about this screening offer.

Methods: The PRENID-scale was developed with medical and psychological experts and consists of a knowledge-scale (range 0-25), testing knowledge of trisomy phenotypes and test characteristics, and a deliberation-scale (range 39-195) measuring the process of thinking about the consequences of (not) engaging in FTS and imagining the consequences of an abnormal result, such as continuing or ending one's pregnancy.

Results: In this pilot study among pregnant women $(\mathrm{N}=$ 23), both the knowledge- and deliberation-scales possessed a high internal consistency reliability (respectively $\alpha=0.83$ and $\alpha=0.89$ ). Women choosing the $\mathrm{CT}$ had highest ( $\mathrm{M}=$ 19.8), whereas women not opting for FTS had lowest 
knowledge scores $(\mathrm{M}=14.7)(p=n s)$. Women opting for both NIPT and CT deliberated most extensively about their choice $(M=167)$, whereas women declining FTS had deliberated least $(\mathrm{M}=128.3)(p=.03)$.

Conclusion: The PRENID-scale is the first scale extensively measuring deliberation in the low-risk pregnant population. We found that women wanting their pregnancy examined more extensively, had more knowledge and deliberation than women who do not, or less extensively, want their pregnancy examined. An informed decision, based on sufficient knowledge and deliberation, was made by $43.5 \%$ of women. Whether women declining FTS make informed decisions according to the PRENID-scale will be investigated in a larger study.

I.M. Bakkeren: None. M.G. Polak: None. F.K.C. Kisters: None. R.H. Galjaard: None. A. Tibben: None. S.R. Riedijk: None.

\section{EPL6.5}

Short and long-term psychological impact of an active GPprovided couple-based ECS test-offer in the Dutch general population

J. Schuurmans ${ }^{1,2}$, M. Plantinga ${ }^{1}$, A. Lucassen ${ }^{2}$, A. Fenwick ${ }^{2}$, K. Abbott ${ }^{\text {, E. Birnie }}{ }^{I}$, I. van Langen ${ }^{1}$, A. Ranchor ${ }^{3}$

${ }^{1}$ Department of Genetics, University Medical Center Groningen/ University of Groningen, Groningen, Netherlands, ${ }^{2}$ Clinical Ethics and Law, University of Southampton, Southampton, United Kingdom, ${ }^{3}$ Department of Health Psychology, University Medical Center Groningen/University of Groningen, Groningen, Netherlands

Introduction: Expanded carrier screening (ECS) could inform reproductive decisions for couples when both partners carry the same autosomal recessive condition. We implemented our new GP-provided couple-based (free) ECS-test for 50 serious recessive conditions (carrier-couple risk: 1 in 150). We investigated short and long term psychological impact (presented here) as well as uptake and feasibility.

Methods: GPs from nine practices invited 4295 female patients aged 18-40 to participate in a longitudinal survey. Inclusion criteria: having a (male) partner, planning children and not being pregnant. GP-provided pre-test counseling was required prior to accepting the test. Psychological impact was measured as: 1) anxiety (6-item STAI, 4-point likert-scale; prorated score range 20-80) and 2) worry of being a carrier-couple (6-items, 4-point likert-scale). Data were compared between female test-acceptors and test nonacceptors and over time (T0-T3 after six months) (T-tests/ non-parametric tests).
Results: Out of 348 eligible women, 190 participated in the survey and 117 couples accepted the test (all results were normal). 1) Anxiety: mean (SD) acceptors vs nonacceptors T0: 32.2 (8.5) vs $33.6(10.4) . \mathrm{p}=0.35$. T3: 28.4 (8.5) vs 36.4 (11.4). $\mathrm{P}<=0.001$. T0-T3: The STAI-score decreased for acceptors -3.5 (8.1) and increased for nonacceptors $1.8(8.8)(\mathrm{p}=0.003)$. 2) Worry: median (IQR): T0: $6(6-8)$ vs $6(6-7.5) ; \mathrm{p}=0.99$ T3: 6 (6-7) vs $6(6-8) ; \mathrm{p}=$ 0.81 .

The worry-score decreased for acceptors $(0,-1-1)$ and non-acceptors $(0,0-1)(\mathrm{p}=0.33)$. Conclusion: Our study is the first to report on psychological impact of a GP-provided couple-based ECS-offer. Some differences exist between test-acceptors and non-acceptors, which will be further investigated.

J. Schuurmans: None. M. Plantinga: None. A. Lucassen: None. A. Fenwick: None. K. Abbott: None. E. Birnie: None. I. van Langen: None. A. Ranchor: None.

\section{EPL6.6}

Next-generation counseling: a model for non-invasive prenatal screening results disclosure and patient management

\section{A. Arjunan, J. Kostialik, R. Ben-Shachar, B. Denne, G. A. Lazarin, C. Haverty}

\section{Counsyl, South San Francisco, CA, United States}

Non-invasive prenatal screening (NIPS) is increasingly offered to the general population by non-genetics specialists. US guidelines recommend patients with negative and positive results be counseled regarding limitations of testing. As a laboratory that provides a results delivery system, we report how this service is utilized for patients undergoing NIPS.

Upon results availability, providers are notified. If negative, patient is contacted by automated email to access results through a secure portal where she may watch tailored informational videos, request "on-demand" genetic counseling, schedule a later consult, or decline all of the above. If a consultation is elected, a summary is sent to the ordering provider. If results are positive, either the ordering provider or our own genetic counselor contacts the patient directly.

64,501 NIPS results were issued through the system. Of these, 4,352 patients elected genetic counseling. $96.1 \%$ of all tests were for patients who received negative results. Approximately $70 \%$ of patients requested an on-demand consult. Median consultation time was 14 minutes (interquartile range: 4-10 minutes) for positive results and six minutes (interquartile range: 4-9 minutes) for negative 
results. The median patient satisfaction rating for consultations was 5.0/5.0.

Combining web education and tele-counseling, we implemented a service that efficiently manages results disclosure. The majority of patients choosing to schedule a consultation had negative results, demonstrating a desire for post-test genetic counseling irrespective of test results. We describe a scalable means of manifesting medical guidelines on post-NIPS patient management, which is imperative to quality care as uptake grows among the general population.

A. Arjunan: A. Employment (full or part-time); Significant; Counsyl. E. Ownership Interest (stock, stock options, patent or other intellectual property); Significant; Counsyl. J. Kostialik: A. Employment (full or part-time); Significant; Counsyl. E. Ownership Interest (stock, stock options, patent or other intellectual property); Significant; Counsyl. R. Ben-Shachar: A. Employment (full or parttime); Significant; Counsyl. E. Ownership Interest (stock, stock options, patent or other intellectual property); Significant; Counsyl. B. Denne: A. Employment (full or part-time); Significant; Counsyl. E. Ownership Interest (stock, stock options, patent or other intellectual property); Significant; Counsyl. G.A. Lazarin: A. Employment (full or part-time); Significant; Counsyl. E. Ownership Interest (stock, stock options, patent or other intellectual property); Significant; Counsyl. C. Haverty: A. Employment (full or part-time); Significant; Counsyl. E. Ownership Interest (stock, stock options, patent or other intellectual property); Significant; Counsyl.

\section{ES1 Communication of genetic information with and within families}

\section{ES1.1}

Facilitating Parents and their Children's Communication about Genetic Conditions: Techniques and Activities

\section{A. Metcalfe}

\section{Sheffield Hallam University, Sheffield, United Kingdom}

Many parents have difficulty talking to their children about an inherited genetic condition that affects them or their child. There are several reasons for this difficulty but one important factor is that parents fear their own and their children's emotional reaction. We have previously codesigned workshops with parents, children, young people and genetic counsellors, which facilitate family communication about the genetic condition. As part of the design a number of techniques and activities are used to facilitate family communication including sculpting, tree of life, news reporting and goldfish bowl. As part of the symposium, some of the techniques used in the workshops will be discussed in more detail, along with a description of how and why the activities work to improve family communication, and why it is so important. This will lead to a discussion with the audience participants about how some of these techniques might be used in genetic counselling sessions to build patients' confidence in communicating with their family members about their risks of an inherited genetic condition. Relevant recent references: Mendes A, Metcalfe, A, Sousa L, Mile P, Clarke A, Sequeiros J, (2017) Communication of Information about Genetic Risks: Putting Families at the Center. Family Process https://doi.org/ 10.1111/famp.12306 Rowland E, Plumridge G, Considine AM, Metcalfe A (2016) Preparing young people for future decision-making about cancer risk in families affected or at risk from hereditary breast cancer: A qualitative interview study. European Journal of Oncology Nursing. Vol. 25, p915. https://doi.org/10.1016/j.ejon.2016.08.006 SocioPsychological Research in Genomics (SPRinG) Collaborative. Metcalfe A (Lead investigator) (2016) Training Genetic Counsellors to Deliver an Innovative Therapeutic Intervention: Their views and experiences of Facilitating Multi-Family Discussion Groups. Journal of Genetic Counselling https://doi.org/10.1007/s10897-016-0008-0. Socio-Psychological Research in Genomics (SPRinG) Collaborative. Metcalfe A (Lead investigator) Developing an intervention to facilitate family communication about inherited genetic conditions and training genetic counsellors in its delivery (2015) European Journal of Human Genetics advance online publication, 7 October 2015; https://doi.org/ 10.1038/ejhg.2015.215.

\section{A. Metcalfe: None.}

\section{Poster presentations}

\section{EMP1.01A}

Expanded carrier screening for recessive disorders: an analysis of the maturing market

\section{Chokoshvili, D. F. Vears, P. Borry}

\section{KU Leuven, Leuven, Belgium}

Expanded carrier screening (ECS) for a large number of recessive disorders is increasingly available to prospective parents, predominantly through commercial providers. To explore the current ECS landscape, we performed a mixedmethods study which involved reviewing websites of ECS providers and in-depth interviews. We performed a comprehensive, multi-step search of websites of commercial ECS providers in English and collected three types of data for analysis: website text, information brochures/ 
educational materials, and video transcripts. In-depth interviews were conducted with representatives of commercial ECS providers. Data collected through both arms of the study were analyzed using inductive content analysis. Websites of 18 commercial providers met our inclusion criteria and 7 interviews comprise our data set. ECS providers were highly transparent regarding the composition of their tests on their websites, and representatives were willing to discuss ECS test development in detail in the interviews. However, providers differed considerably in their views on how ECS tests should be developed. While most ECS providers had a team of genetic counselors available to support patients before or after testing, genetic counseling was typically optional and provided upon request, largely explained by the providers' efforts to minimize conflicts of interest in genetic counseling. In the interviews, ECS providers also reported increasingly prioritizing commercial partnerships with medical institutions, and utilizing their inhouse databases for research purposes. As ECS is becoming widely available, its providers are strategically positioning themselves to succeed in a maturing market. It is important that ECS providers are mindful of the ethical issues arising in ECS.

D. Chokoshvili: None. D.F. Vears: None. P. Borry: None.

\section{EMP1.02B}

\section{Prenatal diagnosis in NF1: What should be offered?}

B. García-Jiménez ${ }^{1}$, A. Ros $^{1}$, E. Castellanos ${ }^{1}$, F. Roca-Ribas ${ }^{1}$, E. Amilibia ${ }^{1}$, A. Castillo ${ }^{1}$, J. Becerra ${ }^{1}$, I. Bielsa ${ }^{1}$, A. Plana ${ }^{1}$,

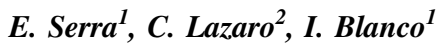

${ }^{1}$ Hospital Germans Trias i Pujol, Badalona, Spain, ${ }^{2}$ Hospital Germans Trias i Pujol, Hospital Germans Trias i Pujol, Spain

We present the case of a couple who decided to carry on a prenatal diagnosis due to the husband was affected by Neurofibromatosis type 1 (NF1). The genetic test identified a pathogenic genetic variant in NF1 gene (c.2033_2034insC) responsible of his disease but finally, the couple rejected to perform the prenatal diagnosis. At her birth, it was ordered the direct genetic test which showed that she was not a carrier. In her paediatrician follow-ups were identified more than six cafe au lait spots suggestive of NF1. Therefore, the complete genetic test of NF1 gene was performed, identifying the presence of a de novo pathogenic variant (c.6792C $>\mathrm{A}$ ).

The co-existance of two or more different mutations is highly unlikely to be found in a family affected by an autosomal dominant disease. However, it has been reported an unusual frequency of families affected of NF1 with two or more independent mutations in $N F 1$ gen. In our patient cohort, we have observed this fact in 3 of 450 families.

Several possible mechanisms might offer an explanation: a high frequency gene conversion or a defect in DNA repair segregating in the family as well as a highly unstable or mutable NF1 allele. Regardless of the possible explanation, these findings should contribute to increase the awareness of the possibility of independent mutation events in NFI gene among relatives. In the context of prenatal screening, the analysis of the whole NFI gene should be considered instead of only studying the mutation of the affected parent.

B. García-Jiménez: None. A. Ros: None. E. Castellanos: None. F. Roca-Ribas: None. E. Amilibia: None. A. Castillo: None. J. Becerra: None. I. Bielsa: None. A. Plana: None. E. Serra: None. C. Lazaro: None. I. Blanco: None.

\section{EMP1.03C}

Preimplantation genetic testing (PGT): an acceptable alternative? A retrospective study in couples at risk for cystic fibrosis and haemoglobinopathies

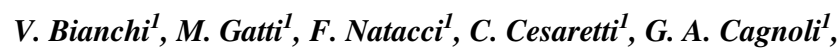
E. Gavazzi ${ }^{1}$, D. Quagliarini ${ }^{2}$, M. Seia ${ }^{3}$, C. Curcio $^{3}$, A. Biffignardi ${ }^{3}$, L. Porcaro ${ }^{3}$, G. Zuliani ${ }^{2}$, A. Riccaboni ${ }^{4}$, L. Restelli, E. Somigliana ${ }^{4}, F$. Lalatta ${ }^{1}$

${ }^{1}$ Clinical Genetics Unit, Fondazione IRCCS Cà Granda, Ospedale Maggiore Policlinico, Milan, Italy, ${ }^{2}$ Department of Obstetrics and Gynecology, Fondazione IRCCS Cà Granda, Ospedale Maggiore Policlinico, Milan, Italy, ${ }^{3}$ Medical Genetics Laboratory, Fondazione IRCCS Ca' Granda Ospedale Maggiore Policlinico, Milan, Italy, ${ }^{4}$ Infertility Unit, Fondazione IRCCS Ca' Granda, Ospedale Maggiore Policlinico, Milan, Italy

Introduction: We analyzed a cohort of couples who participated in a genetic counselling session to prepare them for prenatal diagnosis (PD) or preimplantation genetic testing (PGT). Materials and Methods: We conducted a retrospective study, over a three-year period (January 2015 to December 2017). From a total of 620, we selected 244 couples, 181 at risk for haemoglobinopathies (Hbpathies) and 63 for cystic fibrosis $(\mathrm{CF})$. We have chosen these two autosomal recessive diseases because of their high prevalence in Italy and because our Institution offers a comprehensive service for both diseases which includes a paediatric and adult clinical centre, a prenatal and an infertility unit and an on-site genetic laboratory for both reproductive options.

Results: We compared the 92 couples who chose PGT versus the 152 couples that opted for PD. Among the $63 \mathrm{CF}$ 
couples $43 \%$ chose PD and $57 \%$ opted for PGT, while $69 \%$ of Hbpathies couples requested DP and 31\% PGT.

Conclusions: The aim of our study is to find indicators (clinical, genetic, socio-demographic) that can predict the choice of PD versus PGT, in order to personalise genetic counselling and to find ways to rationalize scarce resources based on the real needs of at risk couples. To the best of our knowledge this is the first Italian study that analyse such a homogeneous cohort (the same clinical entities, time span, hospital, and full resources and opportunities). Moreover having two significative cohorts for both diseases, we could further dissect the data between pathologies and within the same pathology.

V. Bianchi: None. M. Gatti: None. F. Natacci: None. C. Cesaretti: None. G.A. Cagnoli: None. E. Gavazzi: None. D. Quagliarini: None. M. Seia: None. C. Curcio: None. A. Biffignardi: None. L. Porcaro: None. G. Zuliani: None. A. Riccaboni: None. L. Restelli: None. E. Somigliana: None. F. Lalatta: None.

\section{EMP1.04D}

The experience of Preimplantation Genetic Diagnosis (PGD) among Muslim couples in Oman in the Middle East

\author{
K. Al-Kharusi ${ }^{1}$, Z. Bruwer ${ }^{1}$, T. Wessels ${ }^{2}$ \\ ${ }^{1}$ Sultan Qaboos University Hospital, Muscat, Oman, \\ ${ }^{2}$ University of Cape Town, Cape Town, South Africa
}

Introduction: Preimplantation genetic diagnosis (PGD) is an alternative reproductive technology integrated with invitro fertilisation (IVF). It is a well-established technique offering a reproductive option for families at a high risk of transmitting a genetic disorder, allowing them to avoid termination of pregnancy (TOP). Traditional prenatal testing involves testing fetal cells with the option of TOP of an affected fetus. In Arab communities where TOP is restricted under the majority of Muslim law, invasive testing for this purpose is not preferable. For these couples and their family members, PGD is a feasible option as the fetus is diagnosed before implantation and allows for only healthy embryos to be implanted.

Materials and method: This qualitative study utilised a phenomenological approach to explore the experience of Omani families who had selected to undergo PGD as a means of reducing the risk of having a child affected with a genetic disorder. Fourteen participants from eight families who underwent PGD were interviewed. Data collected were transcribed verbatim and analysed using thematic analysis.

Results: The research identified four main themes; Anxiety "Taraqub"; Unforeseen; Secrecy; Me and My Partner. Some participants were confronted with unseen procedural aspects that contradict with their religious and cultural respcts. Overall, although participants found the experience stressful and time consuming it remained their favored option. Conclusion: The findings of the research have provided insight into the PGD experiences of Omani families. These provide some unique cultural and religious perspectives that can be considered when counselling Omani, Arab and Muslim couples.

K. Al-Kharusi: None. Z. Bruwer: None. T. Wessels: None.

\section{EMP1.05A}

Do couples who opt for preimplantation genetic diagnosis get pregnant spontaneously?

\section{Slegers, C. Winter, K. Van Berkel, B. Dimitrov, K. Keymolen, M. Bonduelle, J. Nekkebroeck}

Centre for Medical Genetics, Brussels, Belgium

Introduction: Prenatal diagnosis (PND) and preimplantation genetic diagnosis (PGD) play an important role in reproductive choice for couples who are at risk of transmitting a genetic disorder. However, choosing between PND and PGD appears to be complex. The goal of this research project is to explore whether and why couples are switching between PND and PGD; in this specific analyses we focus on couples who chose PGD and still got pregnant spontaneously.

Methodology: An explorative retrospective analysis was performed on non-pregnant Belgian couples who chose to start a PGD treatment at the Centre for Medical Genetics of the UZ Brussel between January 2016 and September 2017.

Results: Out of 425 couples, 30 couples (7\%) got pregnant spontaneously. Across these 30 couples, $46 \%$ got pregnant prior to a first PGD attempt i.e. during the three month test development, 53\% performed at least one PGD attempt. Only one couple planned their pregnancy, in contrast to nine couples who got pregnant unexpected. The motivation for the twenty other couples is unknown. In total, seventeen out of thirty couples ended up doing PND. Among the remaining thirteen couples, only two couples declined PND and one couple reported a miscarriage. The continuation of the other couples $(\mathrm{n}=10)$ is unknown.

Conclusion: These preliminary data confirm that couples are intentionally or not switching from PGD to a spontaneous pregnancy, despite their genetic risk. However, future research is needed to clarify the motivation for switching since this could have an influence on the clinical practice of genetic counselors.

I. Slegers: None. C. Winter: None. K. Van Berkel: None. B. Dimitrov: None. K. Keymolen: None. M. Bonduelle: None. J. Nekkebroeck: None. 


\section{EMP1.06B}

Looking back A retrospective study of the unaffected BRCA1 \& 2 testing completed by the Cheshire \& Merseyside Clinical Genetics Service from July 2013 March 2017

\section{J. J. Bussell, D. L. Melville}

Cheshire \& Merseyside Clinical Genetics Service, Liverpool, United Kingdom

In the Cheshire \& Merseyside Clinical Genetics Service we provide genetic counselling to a population of 2.8 million. In 2013 the National Institute of Health and Care Excellence (NICE) released recommendations for the implementation of genetic testing of the BRCA1 \& BRCA2 genes for unaffected individuals with a family history of cancer:

'Offer genetic testing in specialist genetic clinics to a person with no personal history of breast or ovarian cancer if their combined BRCA1 and BRCA2 mutation carrier probability is $10 \%$ or more and an affected relative is unavailable for testing,

This project reflects back on our application of this guideline within our service from 2013 - 2017 and evaluate our compliance with N.I.C.E guidance when offering unaffected BRCA testing to our patients.

We found that of 157 unaffected BRCA tests performed in our service between, there was an average Manchester score of 25. The BRCA mutation and Variant of Uncertain Significance (V.U.S) combined pick-up rate was $22.29 \%$ and there does not appear to be any significant trends in the amount of tests that were completed. Additionally there was an incidental CHEK2 pick-up rate of $1.91 \%$. We found that 21 patients had $<10 \%$ BRCA probability and therefore did not meet the N.I.C.E recommendations. The reasons for this included Jewish ancestry, small family size and unconfirmed familial testing.

\section{REFERENCES:}

1. N.I.C.E. (2013). Familial breast cancer: classification, care and managing breast cancer and related risks in people with a family history of breast cancer. Available: https://www.nice.org.uk/guidance/cg164/chapter/

Recommendations. Last accessed 9th February 2018.

\section{J.J. Bussell: None. D.L. Melville: None.}

\section{EMP1.07C}

Exploring the views of parents of children affected by phenylketonuria or medium chain acyl CoA dehydrogenase deficiency on extending the number of metabolic conditions included in the Newborn Blood Spot Screening Programme in England and Wales
H. McMillan ${ }^{1,2}$, T. Austin ${ }^{1,3}$, T. Clancy $^{1,2}$

${ }^{1}$ University of Manchester, Manchester, United Kingdom,

${ }^{2}$ Manchester Centre for Genomic Medicine, Manchester, United Kingdom, ${ }^{3}$ West Midlands Regional Genetics Service, Birmingham, United Kingdom

Introduction: Phenylketonuria (PKU) and medium chain acyl CoA dehydrogenase deficiency (MCADD) are included on the newborn blood spot (NBS) screening programme in England and Wales. Pressure exists from advocacy groups, clinicians and researchers to include more conditions. Others stress the need for a comprehensive assessment of benefits, harms and costs before adding any conditions. Taking account of parents' views about this is essential because they and their children are directly affected.

Methodology: Parents of children with PKU or MCADD were ascertained through purposive sampling and semistructured interviews were conducted. Transcripts were analysed using interpretive phenomenological analysis.

Results: 7 of 29 eligible parents consented to participate. All described a parental duty to maximise the health of the child as the reason for accepting screening. But there was limited engagement with standardised information provision. Parents perceived screening as routine and had expected a normal result. All supported an expansion of the screening programme, advocating recognition of the broader benefits of early diagnosis to families.

Discussion: Our findings show some divergence between parental views and those of health professionals on the NBS screening programme. Any further expansion should be preceded by a review of the efficacy of current information provision, as well as more qualitative explorations of how couples can be supported during the diagnostic period.

H. McMillan: None. T. Austin: None. T. Clancy: None.

\section{EMP1.08D}

Telegenetic counselling consultations - A qualitative study of critical implementation factors, according to health care professionals in Sweden

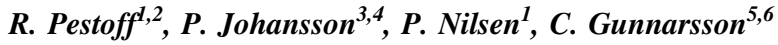

${ }^{1}$ Division of community medicine, Department of Medical and Health Sciences, Faculty of Medicine Health Sciences, Linköping, Sweden, ${ }^{2}$ Centre for rare diseases in Southeast region, Linköping, Sweden, ${ }^{3}$ Department of social and welfare studies, Linköping University, Linköping, Sweden,

${ }^{4}$ Department of Internal Medicine and Department of Medical and Health Sciences, Linköping University, Norrköping,

Sweden, ${ }^{5}$ Department of Clinical and Experimental Science, Linköping University, Department of Genetics, Linköping, 
Sweden, ${ }^{6}$ Centre for rare diseases in Southeast region of Sweden, Linköping, Sweden

This research focused on an alternative mode of delivery for genetic counselling in Sweden. The aim was to explore perceptions of telegenetic counselling consultations among health care professionals (HCPs) in order to identify crucial factors for implementation in Swedish Health care. Telegenetic counselling is the use of videoconferencing to provide genetic counselling. Due to factors such as lack of professional genetic counsellors, vast geographical distances, and physical barriers genetic counselling is not always offered to patients in need. Qualitative, semistructured interviews were performed with 16 genetic counselling providers, in the Southeast healthcare region in Sweden, and a phenomenographic approach was applied. Significant exerts were identified in each transcript, which led to subcategories that supported the main categories. The findings reveal three main categories: Perceived requirements for optimal use; Impact on clinical practice and Patient benefits. Benefits were identified for both patients and HCPs, but also specific barriers to overcome before this mode of delivery is introduced into the clinical genetic counselling setting. The findings from this study could improve the use and accessibility of genetic counselling in Sweden. However, also the perceptions of patients will need to be sought in further exploratory research to include as many critical factors as possible.

R. Pestoff: None. P. Johansson: None. P. Nilsen: None. C. Gunnarsson: None.

\section{EMP1.09A}

\section{Genetic Counselling Service in tertiary Hospital of} Catalonia, experience for the first 3 years

\section{N. Capdevila, M. Guitart, N. Baena, A. Ruiz, E. Gabau}

\section{Parc Taulí Sabadell, University Hospital, Sabadell, Spain}

Introduction: Genetic counselling is considered an integral part of medical care for patients with genetic diseases in developed countries.

In Spain, genetic counselling is recognized in the Bioethical Law since 2006, but its implementation is still in progress.

The Genetics Unitin our hospital was consolidated in 1985. It was made up by a biologist specialized in laboratory genetics and a pediatrician specialized in clinical genetics who also offered genetic counselling. In January 2015, a genetic counsellor was incorporated into the Genetics Unit starting an specific genetic counseling service.
Materials and Methods: We have collected data about the kind of and number of genetic counselling consultations during the first three years of our genetic counseling service.

Genetic counselling consultations have been differentiated according to first visits and successive ones. Furthermore, these consultations has been classified into: preconception and prenatal focused on the reproductive genetic counseling, pediatric consultation related to the genetic counseling about family repercussions of genetic diseases on its children and adult consultation based on diagnosis confirmation and family studies.

\begin{tabular}{llllll}
\multicolumn{1}{c}{ Results: } & & & & & \\
\hline Genetic Counseling & Total $^{\circ}$ & $\mathrm{N}^{\mathrm{o}}$ & Preconception and prenatal & Paediatrics & Adults \\
\hline First & \multirow{2}{*}{305} & 205 & 119 & 28 & 58 \\
Successive & & 100 & 58 & 14 & 28 \\
First & 341 & 241 & 135 & 52 & 54 \\
Successive & & 100 & 56 & 22 & 22 \\
First & 445 & 372 & 137 & 94 & 141 \\
Successive & & 73 & 37 & 14 & 22 \\
\hline
\end{tabular}

Conclusions: During the first three years of genetic counselling service implementation, there has been an exponential increase in demand. This fact demonstrates the good acceptance of genetic counselling from other services in our hospital

N. Capdevila: None. M. Guitart: None. N. Baena: None. A. Ruiz: None. E. Gabau: None.

\section{EMP1.10B}

A new model of care for women at average to moderate risk of familial breast cancer

\section{K. J. Storey, E. Higgs, R. Purvis, M. Kentwell, T. Schenberg, Y. Antill}

Parkville Familial Cancer Centre, Parkville, Australia

Introduction: Due to increased awareness and reduced test costs, referrals for advice and genetic testing for familial breast cancer (FBC) have increased in recent years. Many referrals are for women at average to moderate risk for FBC. Additional pressure to already busy genetic services has required consideration of novel solutions.

Method: A group seminar was offered to women from families assessed as non-high risk for FBC, while minimising impact on waitlists for families at high risk. Seminars covered a range of issues including: factors known to influence breast cancer risk (including non-genetic), breast cancer genetic risk assessment, self-funded genetic testing, and psychosocial issues commonly arising in families affected by cancer. Brief consults with a genetics staff member were available directly after the seminar to discuss 
any issues specific to the individual. Attendees could choose to proceed with genetic testing on the day.

Results: Since April 2015, 5 seminars have been conducted with a total of 46 attendees from 40 families (between 6 and 15 attendees per seminar). Individual consults were requested by $41(89 \%)$ attendees. A third $(33 \%)$ proceeded with privately funded genetic testing. Of the results available, no mutations were identified. Feedback forms were gathered from attendees of 4 seminars. Of the $27(63 \%)$ responses, $26(96 \%)$ reported their knowledge improved, 25 (93\%) indicated their needs were met, and 17 (63\%) found their perception of breast cancer risk reduced. Conclusion: This streamlined approach has been beneficial for patients while minimising the impact on our genetics service.

K.J. Storey: None. E. Higgs: None. R. Purvis: None. M. Kentwell: None. T. Schenberg: None. Y. Antill: None.

\section{EMP1.11C}

Making genetic counselling services accessible - Results of a pilot service delivery feasibility study of telemedicine appointments

\section{J. J. Bussell, D. L. Melville}

Cheshire \& Merseyside Regional Clinical Genetics Service, Liverpool, United Kingdom

We provide genetic counselling to a population of 2.8 million, including 80,000 on the Isle of Man. Literature from the US and the Netherlands suggests that telegenetic consultations could be an alternative to face to face consultations.

This small pilot study aimed to test the feasibility and acceptability of telemedicine services. A European study showed that only $9 \%$ of genetics professionals currently use telemedicine facilities (Otten et al., 2016). It is unknown how widespread this practice is within the UK.

Of 60 patients offered a telemedicine appointment, six accepted. Advantages included convenience for the patient and reduction in travel. Reduced quality of interaction and technology problems arose and these findings are consistent with Buchanan et al., (2015) and Otten et al., (2016). Of 21 patients asked, 14 felt telemedicine was an acceptable alternative, however 7 did not.

In conclusion, telemedicine appointments stimulated mixed reviews. But in order to be a viable alternative, the hosting IT system needs to be robust and clinicians require relevant training and support. Overcoming these problems would enhance the acceptability to patients and clinicians.

\section{References}

Buchanan AH, Datta SK, Skinner CS, Hollowell GP, Beresford HF, Freeland T, Rogers B, Boling J, Marcom PL and Adams MB (2015) Randomized Trial of Telegenetics vs. In-Person Genetic Counseling: Cost, Patient Satisfaction and Attendance. J Genet Couns. 24(6): 961-70.

Otten E, Birnie E, Lucassen AM, Ranchor AV and Van Langen IM (2016) Telemedicine Uptake Among Genetics Professionals in Europe: Room for Expansion. Eur J Hum Genet 24(2): 157-63.

J.J. Bussell: None. D.L. Melville: None.

\section{EMP1.12D}

Evaluation of a centralised national telephone genetic counselling service that facilitates $B R C A 1 / 2$ testing for women with recurrent high-grade serous ovarian cancer

L. E. Forrest ${ }^{1,2}$, J. McKinley ${ }^{1}$, R. Forbes Shepherd ${ }^{1,2}$, V. Rasmussen ${ }^{1}$, P. A. James ${ }^{1,2}$, B. Meiser ${ }^{3,4}$, M. Young ${ }^{5}$

${ }^{1}$ Parkville Familial Cancer Centre, Peter MacCallum Cancer Centre, Melbourne, Australia, ${ }^{2}$ Sir Peter MacCallum Department of Oncology, The University of Melbourne, Melbourne, Australia, ${ }^{3}$ Prince of Wales Clinical School, The University of New South Wales, Sydney, Australia, ${ }^{4}$ Psychosocial Research, Hereditary Cancer Clinic, Prince of Wales Hospital, Sydney, Australia, ${ }^{5}$ Genome One, The Garvan Institute of Medical Research, Sydney, Australia

Introduction: Women with recurrent high-grade serous ovarian cancer (HGSOC) with germline BRCA1/2 mutations benefit from poly (ADP-ribose) polymerase (PARP) inhibitors. Prompt access to genetic testing is essential to guide treatment decisions, yet uptake of BRCA1/2 testing by Australian women with HGSOC remains low. In 2016, a national centralised telephone genetic counselling (TGC) service for women with HGSOC was established to address barriers to BRCAl/2 testing in Australia. This study examined how women with HGSOC experienced receiving TGC. Methods: 213 (75\%) women with recurrent HGSOC who used the TGC service were invited to participate in a survey. Survey domains included accessibility of genetic services, acceptability and experiences of TGC, and decision-making about BRCAl/2 testing.

Results: 107 women (48\%) responded to the survey; $7.5 \%$ tested positive for a BRCAI/2 mutation. Mean time since genetic testing was 6.0 months (0.4-18.6 months). Women predominantly used TGC to inform clinical management of their cancer and to overcome geographical barriers to their local genetics service, and were highly satisfied with the TGC content, process, and timing. However, their preferred provider for BRCAI/2 testing was their oncologist. The majority (64\%) made informed choices regarding genetic testing. Overall, women reported limited impact from results disclosure after genetic testing. 
Nineteen women reported severe ovarian cancer-related distress.

Conclusions: TGC is an acceptable service to mainstream treatment focussed $B R C A 1 / 2$ genetic testing for women with recurrent HGSOC. This service overcame geographical barriers impeding access to genetics services ensuring BRCA1/2 testing was facilitated expediently to inform treatment. Funding: NHMRC program grant (APP 1092856).

L.E. Forrest: None. J. McKinley: None. R. Forbes Shepherd: None. V. Rasmussen: None. P.A. James: None. B. Meiser: None. M. Young: None.

\section{EMP1.13A}

Parent's understanding and attitudes towards therapeutic trials in Rett syndrome

\section{S. Russ, A. J. Clarke \\ Institute of Medical Genetics, Cardiff, United Kingdom}

Novel 'rational' treatments are being developed for numerous rare diseases, including Rett syndrome (RTT). Therapeutic trials will be required to determine the safety and efficacy of these treatments but face significant challenges from the small pool of patients available to participate and will raise issues of ethics and communication.

Semi-structured interviews were conducted with parents of patients with RTT $(\mathrm{n}=10)$ to explore their (hypothetical) responses to an invitation to participate in a trial.

Parents thought clinical trials were important but multiple factors would impact on their decision. Families also experienced tension between their wish to participate in trials and the practical/emotional barriers, especially if an affected daughter's health could be described as fragile and vulnerable. Parents often demonstrated their competing sense of 'responsibilities' as both the parent of an affected daughter and as a member of the RTT community. Among parents of younger affected girls in the cohort (aged 11-18 years), there was also some evidence of therapeutic misconception.

Awareness of the family perspectives on clinical trials will be important in enabling recruitment. This will involve (i) using a cross-over trial design, where feasible, (ii) minimising requirement for hospital admission as part of the trial, and (iii) taking care to minimise the sense of tension set up by an invitation to participate in a clinical trial. This will mean taking care not to inflate the expectations of what the treatment under trial is likely to achieve. This may reduce barriers to recruitment and improve family experiences of participation.

S. Russ: None. A.J. Clarke: None.

\section{EMP1.14B}

Does the type of disease matter when receiving secondary findings? A qualitative vignette study on Finnish adults' views

M. Vornanen ${ }^{1}$, K. Aktan-Collan ${ }^{1}$, N. Hallowell ${ }^{2}$, H. Konttinen ${ }^{1}$, A. Haukkala ${ }^{1}$

${ }^{1}$ University of Helsinki, Helsinki, Finland, ${ }^{2}$ University of Oxford, Oxford, United Kingdom

Background: Validated, clinically actionable secondary findings of genomic sequencing (SFs) are recommended to be reported back to consented patients/research participants. Professionals have discussed how to categorize SFs when asking for consent. To create SF reporting practices that best serve people's needs, public's perspectives need to be integrated in these discussions.

Methods: We performed a qualitative vignette study among Finnish adults. Participants were asked to imagine themselves receiving a letter that revealed a SF predisposing to a severe but actionable disease - familial hypercholesterolemia, long QT syndrome, Lynch syndrome, or Li-Fraumeni syndrome. Participants wrote down their initial reactions to receiving this hypothetical information $(\mathrm{N}=29)$, and then attended focus group discussions $(\mathrm{N}=$ 23). Written reactions and transcribed focus group discussions were analysed using inductive thematic analysis.

Results: Written first reactions to hypothetical SFs varied according to perceived severity and familiarity of disease. Focus groups considered cancer related SFs more threatening than cardiovascular related SFs, but less distressing than genetic risks for psychiatric or neurological disorders. In addition to actionability, illness severity in terms of lived experience, access to available treatment, and individual's responsibility to control risk were perceived to vary across diseases and influence how threatening knowing one's genetic risk would be.

Conclusion: In addition to clinical validity and utility, SF consent and reporting practices need to take into account potential familiarity and lay illness representations of different diseases. (Funding: Academy of Finland, grant 275033 to AH.).

M. Vornanen: None. K. Aktan-Collan: None. N. Hallowell: None. H. Konttinen: None. A. Haukkala: None.

\section{EMP1.15C}

How are modern gene therapies portrayed to the public? A content analysis of selected newspaper coverage concerning the approval of Kymriah in the US, Germany and Switzerland 
N. Aebi ${ }^{1}$, H. Meyer zu Schwabedissen ${ }^{1}$, B. Zimmermann ${ }^{2}$

${ }^{1}$ Biopharmacy, Department of Pharmaceutical Sciences, Basel, Switzerland, ${ }^{2}$ Institute for Biomedical Ethics, Basel, Switzerland

Introduction: On 30 August 2017, Kymriah (launched by Novartis) became the first gene therapy approved by the US Food and Drug Administration (FDA) for the treatment of children and young adults suffering from Acute Lymphocystic Leucemia (ALL). The aim of this study was to analyze how print media informed the public about Kymriah by exploring which aspects of the therapy were emphasized in media coverage and whether the assessment of gene therapies by media changed after the FDA approval.

Methodology: We qualitatively analyzed print media articles about gene therapy in 2017 using thematic analysis. The analysis was focused on broadsheet newspapers from the US, Germany and the German-speaking part of Switzerland.

Results: Key aspects in print media coverage on gene therapy shifted from good medical results and side effects towards the issue of high treatment costs after Kymriah's FDA-approval. Media reporting about Kymriah was most profound in the US, but merely present in Germany. In Switzerland, the focus was on high treatment costs and success-rates.

Conclusions: The public was exposed to a rather skeptical reporting, focusing more on treatment costs or side effects of Kymriah than on the medical advantages of healing ALL. This was most extreme in Switzerland, where Novartis' headquarter is situated. Despite the upcoming submission of Kymriah's approval in the European Union, the subject of gene therapies was not discussed extensively in German newspapers compared to the other countries. Unsurprisingly, reporting was broadest in the US where the therapy got approved.

N. Aebi: A. Employment (full or part-time); Modest; Wettstein Apotheke (pharmacist assistant 30\%). H. Meyer zu Schwabedissen: None. B. Zimmermann: None.

\section{EMP1.16D}

Opinions of young hearing people living in the Sakha Republic (Russia) about potential risk of the birth of deaf child

A. M. Cherdonova ${ }^{1}$, A. V. Solovyev ${ }^{1,2}$, V. G. Pshennikova ${ }^{1,2}$, N. A. Barashkov ${ }^{1,2}$, G. P. Romanov ${ }^{1,2}$, A. A. Nikanorova ${ }^{2}$, S. K. Kononova $^{1,2}$, O. L. Posukh ${ }^{3,4}$, S. A. Fedorova ${ }^{1,2}$, F. M. Teryutin $^{1,2}$

${ }^{1}$ Institute of Natural Sciences, Yakutsk, Russian Federation,

${ }^{2}$ Yakut Scientific Center of Complex Medical Problems,
Yakutsk, Russian Federation, ${ }^{3}$ Federal Research Center Institute of Cytology and Genetics, Novosibirsk, Russian Federation, ${ }^{4}$ Novosibirsk State University, Novosibirsk, Russian Federation

Main cause of congenital deafness in the Sakha Republic is recessive mutation c. $-23+1 \mathrm{G}>\mathrm{A}$ in the $G J B 2$ gene. The c.$23+1 \mathrm{G}>\mathrm{A}$ carrier frequency among Yakut population is extremely high (10\%) [Barashkov et al., 2016] and the probability of the birth of deaf child by both hearing parents (with unknown GJB2 status) according to recessive type of inheritance may be estimated as $0.25 \%$. We developed specialized questionnaire consisted of 21 questions concerning genetic testing of deafness. 304 young people (mean age 21 years) were involved in the survey. This study aims to evaluate the awareness of young hearing people about existence of "genetic deafness", their understanding of peculiarities of deafness inheritance, and their opinions about hypothetical risk of the birth of deaf child. For this purpose we analyzed the respondents' answers to appropriate questions from questionnaire. Respondents' answers were as follows: "Is deafness a hereditary disease?" - "yes" $62.2 \%$, "no" - 36.5\%, no response - $1.3 \%$; "Is there a probability of birth of deaf child by hearing parents?" "yes" - 83.9\%, "no" - 15.1\%, no response - 1.0\%; "Is there a probability of birth of deaf child by you?" - "I don't know" $65.1 \%$, "no" - 29.3\%, "yes"- $4.3 \%$, no response $-1.3 \%$. Thus, the majority of respondents $(83.9 \%)$ admitted a potential risk of the birth of deaf children by hearing parents but only $4.3 \%$ of respondents may attribute this risk to themselves. The study was supported by RFBR (\#18-01300738_A), FASO_BRK0556-2017-0003 and the Project of the MESRF of Russia (\#6.1766.2017).

A.M. Cherdonova: None. A.V. Solovyev: None. V.G. Pshennikova: None. N.A. Barashkov: None. G.P. Romanov: None. A.A. Nikanorova: None. S.K. Kononova: None. O.L. Posukh: None. S.A. Fedorova: None. F.M. Teryutin: None.

\section{EMP1.17A}

Who has a say in mass media coverage about predictive genetic testing? A comparative media content analysis from UK and Switzerland

\section{B. M. Zimmermann ${ }^{1}$, S. Kolb ${ }^{2}$, N. Aebi ${ }^{3}$, B. S. Elger ${ }^{1}$, D. M.} Shaw $^{1}$

${ }^{1}$ Institute of Biomedical Ethics, University of Basel, Basel, Switzerland, ${ }^{2}$ Department of Business Communication, University of applied Sciences - HTW Berlin, Berlin, Germany, ${ }^{3}$ Biopharmacy, Department of Pharmaceutical Sciences, University of Basel, Basel, Switzerland 
Introduction: Predictive genetic testing (PGT) is increasingly used in clinics and by direct-to-consumer genetic testing companies, but is accompanied by important ethical, legal and social issues. A balanced public debate is important to enable lay people to form informed autonomous opinions. Our research question thus was: To what extend is media coverage about PGT balanced by citing a high variety of stakeholders and highlighting both positive and negative aspects? Methodology: We performed a quantitative comparative media content analysis on two Swiss-German and two UK broadsheet newspapers about PGT in the period 2011-2016. Stakeholders that were cited in the coverage were collected, as well as their evaluation of the topics.

Results: A total of 137 articles matched our inclusion criteria, $72 \%$ of which were published in the UK. Most stakeholders cited in coverage were natural scientists and medical doctors, but other experts, i.e., bioethicists or lawyers were rare. Switzerland quoted more politicians, whereas UK coverage more frequently featured statements of affected people. On average, Swiss articles quoted three times more stakeholders than UK articles. Evaluation of PGT was more positive in the UK. Switzerland had more negative and ambivalent articles.

Conclusions: Our findings show that media coverage differs significantly between Switzerland and the UK, illustrating cultural differences. Switzerland, which is considered more conservative and legally restrictive towards genetics, had less coverage but more balanced reporting in terms of amount and variability of stakeholders as well as evaluations compared to the more geneticsfriendly UK, where coverage was more positive.

B.M. Zimmermann: None. S. Kolb: None. N. Aebi: A. Employment (full or part-time); Modest; Wettstein Apotheke Basel, Switzerland (pharmacy assistant, 30\%). B.S. Elger: None. D.M. Shaw: None.

\section{EMP1.18B}

Prenatal chromosomal microarray analysis: A populationbased study of women's attitudes towards reporting of genomic testing results in pregnancy

V. Libman ${ }^{1}$, M. Macarov ${ }^{2}$, Y. Sompolinsky ${ }^{3}$, M. Osovsky, A. Huta Winter ${ }^{2}$, D. Hochner-Celnikier ${ }^{3}$, L. Basel ${ }^{5,6,7,8}$, A. Wiznitzer ${ }^{9,7}$, Y. Neumark ${ }^{1}$, V. Meiner ${ }^{2}$, A. Frumkin ${ }^{2}$, Y. Friedlander ${ }^{1}$, S. Shkedi-Rafid ${ }^{2}$, H. Hochner ${ }^{1}$

${ }^{1}$ Braun School of Public Health, Hebrew University-Hadassah Medical Center, Jerusalem, Israel, ${ }^{2}$ Department of Genetics and Metabolic Diseases, Hebrew University-Hadassah Medical Center, Jerusalem, Israel, ${ }^{3}$ Department of Obstetrics and Gynecology Mount Scopus, Hebrew University-Hadassah Medical Center, Jerusalem, Israel, ${ }^{4}$ Department of
Neonatology, Rabin Medical Center, Petah Tikva, Israel, ${ }^{5}$ The Raphael Recanati Genetics Institute, Rabin Medical Center, Beilinson Hospital, Petah Tikva, Israel, ${ }^{6}$ Pediatric Genetics Unit, Schneider Children Medical Center, Petah Tikva, Israel, ${ }^{7}$ Sackler School of Medicine, Tel Aviv University, Tel Aviv, Israel, ${ }^{8}$ Felsenstein Medical Research Center, Rabin Medical Center, Petah Tikva, Israel, ${ }^{9}$ The Helen Schneider Hospital for Women, Rabin Medical Center, Petah Tikva, Israel

Introduction: Chromosomal microarray analysis (CMA) has largely replaced conventional karyotyping in prenatal testing. The major advantage of CMA is higher detection rate of abnormalities. Nevertheless, CMA also increases the likelihood of identifying susceptibility loci and variants associated with adult-onset conditions. Implementation of CMA in prenatal testing is debated and no international consensus has been reached. This study examined attitudes of women towards reporting of genomic testing results in pregnancy. Methods: Population-based study of women hospitalized in maternity departments. Post-partum interviews were conducted using a computerized multiplechoice questionnaire.

Results: 750 women (75\% response rate) participated in the study. Women's responses clearly differed based on severity, treatment availability and penetrance of conditions. For example, $69 \%$ of women reported an interest in receiving genetic information on severe congenital diseases; $61 \%$ were interested in information on treatable adult-onset diseases, compared to $33 \%$ when treatment is unavailable; $65 \%$ expressed interest in receiving information when risk for autism was $40 \%$ as opposed to $41 \%$ when risk was $10 \%$. Psycho-social characteristics, such as educational attainment, level of religious observance, and degree of concern for newborn's health, were associated with responses (p-values $<0.01$ ). $73 \%$ of participants thought that parents should be able to decide what results to receive.

Conclusions: Participants, representing the population of pregnant women in Israel, are generally interested in receiving information in pregnancy on a range of genetic conditions, yet responses differ by characteristics of condition and respondent. Participants support autonomy of parents in deciding what information they should receive. Funding: Israel NIHP grant 2015/82

V. Libman: None. M. Macarov: None. Y. Sompolinsky: None. M. Osovsky: None. A. Huta Winter: None. D. Hochner-Celnikier: None. L. Basel: None. A. Wiznitzer: None. Y. Neumark: None. V. Meiner: None. A. Frumkin: None. Y. Friedlander: None. S. Shkedi-Rafid: None. H. Hochner: None.

\section{EMP1.19C}

The attitudes of gamete and embryo donors towards the genetic screening of donors 
N. Somanathan ${ }^{1}$, D. Amor', S. Lewis ${ }^{2}$

${ }^{1}$ University of Melbourne, Melbourne, Australia, ${ }^{2}$ Murdoch

Children's Research Institute, Melbourne, Australia

Background: Chromosomal karyotyping and carrier screening are two different forms of genetic screening used within IVF clinics, to test donors for chromosomal abnormalities and common single gene disorders. To date, there is no research available that explores the opinions of the donors towards the current and future genetic screening practices.

Method: Nine gamete and embryo donors in total were interviewed about their journey in choosing to donate, their experiences of completing the donor information and genetic health questionnaires and their attitudes towards current and future genetic screening practices within IVF.

Results: The donors were comfortable answering the Genetic Health Questionnaires (GHQ) and understood its significance for recipients. The majority of participants found the current genetic screening practices important. In regards to future screening, all the participants were apprehensive about advanced genomic technologies such as: Whole Genome Sequencing (WGS) and Whole Exome Sequencing (WES). Their main concerns focused on the disclosure of their results and whether these technologies would lead to the creation of designer babies.

Conclusion: This study suggests that gamete donors understand the need for genetic screening; however, there is a misconception about the use of genomic technologies, suggesting an educational need amongst the public. If genomic technologies were to be introduced into IVF centers for the screening of donors, then health professionals need to consider whether this type of screening should be targeted towards well understood disease, that benefit from treatment or screening.

N. Somanathan: None. D. Amor: None. S. Lewis: None.

\section{EMP1.20D}

Does optimal size of cancer pedigrees depend on degree of relatedness or the number of individuals?

V. Stefansdottir ${ }^{1,2}$, H. Skirton ${ }^{3}$, G. Olafsdottir ${ }^{4}$, O. T. Johannsson $^{5}$, H. Olafsdottir ${ }^{6}$, L. Tryggvadottir ${ }^{4}$, J. J. Jonsson ${ }^{7,2,6}$

${ }^{1}$ Landspitali National University Hospital, Reykjavik, Iceland, ${ }^{2}$ Dept. of Biochemistry and Molecular Biology, Univ. of Iceland, Reykjavik, Reykjavik, Iceland, ${ }^{3}$ Faculty of Health and Human Sciences, University of Plymouth, Plymouth, United Kingdom, ${ }^{4}$ Icelandic Cancer Registry, Reykjavik, Iceland, ${ }^{5}$ Dept. Of Medical Oncology, Landspitali - National University Hospital, Reykjavik, Iceland, ${ }^{6}$ Genetical committee of the
University of Iceland and Landspitali, Reykjavik, Iceland, ${ }^{7}$ Dept. of Genetics and Molecular Medicine, Landspitali National University Hospital,, Reykjavik, Iceland

Introduction: In the Icelandic genetics clinic we use a genealogy database and nationwide cancer registry to construct electronic mega-pedigrees. Experience with HBOC work suggests that $3^{\circ}$ pedigrees have optimal efficiency, but is this dependent on number of individuals?

Methods: The study group comprised randomly chosen 209 women from the genetics clinic: 104 positive and 105 negative for the founder pathogenic BRCA2: c.771_775delTCAAA. Boadicea to estimate the likelihood of having the PV and MedCalc ${ }^{\mathrm{TM}}$ Receiver Operating Characteristic (ROC) Curve Analysis and C-statistics were used.

Results: To further evaluate the relationship between the size of the pedigree and efficiency of risk assessment, we divided the $3^{\circ}$ pedigrees into two groups ranked on the basis of number of individuals in each pedigree. The lower number group $(\mathrm{n}=105$, average no. of individuals 30 , range $=9$-46) had a C-statistic of 0.718 , but the higher number group $(n=104$, average no. of individuals 133 , range $=46-220$ ) had a C-statistic of 0.822 . We divided the $4^{\circ}$ pedigrees in two according to size. The lower number group ( $n=103$, average no. of individuals 56 , range $=15$ 87) had a C-statistic of 0.719 , but the higher number group ( $n=103$, average no. of individuals 145 , range 87-257) had a C-statistic of 0.815 . The differences between the groups for $3^{\circ}$ and $4^{\circ}$ relative pedigrees were not statistically significant. We converted the lower number groups in $3^{\circ}$ pedigrees to $4^{\circ}$ relative pedigrees, but there was no improvement in the C-statistic (0.718).

Conclusions: There was no gain of efficiency by including $4^{\circ}$ relatives.

V. Stefansdottir: None. H. Skirton: None. G. Olafsdottir: None. O.T. Johannsson: None. H. Olafsdottir: None. L. Tryggvadottir: None. J.J. Jonsson: None.

\section{EMP1.21A}

Hereditary cancer predisposition syndromes: Evaluation on the influence of personality in predictive genetic testing

L. Moreno ${ }^{1}$, T. Ocaña ${ }^{1}$, A. Sánchez ${ }^{1}$, M. Salinas ${ }^{2}$, S. Iglesias ${ }^{2}$, A. Teulé2, J. Peri', F. Balaguer ${ }^{1}$

${ }^{1}$ Hospital Clínic de Barcelona, Barcelona, Spain, ${ }^{2}$ Institut Català d'Oncologia, Hospitalet de Llobregat, Spain

Background: Knowing the carrier status of mutations in cancer predisposing genes has been hypothesized to have a negative effect on psychological well-being. Different published studies have not reached any consensus. Some 
personality traits have been suggested to have a great influence on psychological distress but only two studies have assessed this issue in this population.

Objectives: Assess the psychological impact of genetic testing, evaluate changes in social life and behaviour, and estimate if personality influences the use of medical resources.

Methodology: Ten adults undergoing predictive genetic testing for cancer predisposition syndromes were included between January and March 2017. Demographic information, personality traits, psychological distress, behaviour in some daily activities and medical resources use were collected before testing and two months after results disclosure.

Results and discussion: High pre- and post-test psychological distress was associated to low education levels, having psychopathological history, pursuing testing for offspring, and being recruited at ICO $(\mathrm{p}<0.05)$. It was also associated with high negative affect, detachment, psychoticism and novelty seeking, and low reward dependence, selfdirectiveness, cooperativeness, and persistence $(\mathrm{p}<0.05)$. High post-test distress was also associated with having pretest psychological distress $(\mathrm{p}<0.05)$. It would be important to know our counselees' personality because it gives us the opportunity to know who to offer more support and how to personalize genetic counselling.

Conclusions: Our results suggest that there are some personality traits which can influence psychological distress in individuals undergoing predictive genetic testing. Further studies need to be performed in order to extrapolate these results to this particular population.

L. Moreno: None. T. Ocaña: None. A. Sánchez: None. M. Salinas: None. S. Iglesias: None. A. Teulé: None. J. Peri: None. F. Balaguer: None.

\section{EMP1.22B}

Exploring the psychological impact of conducting population-based family history screening for familial breast cancer

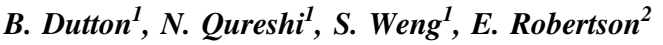 \\ ${ }^{1}$ University of Nottingham, Nottingham, United Kingdom, ${ }^{2}$ FaHRAS, Nottingham, United Kingdom}

Background: Around 5\% of breast cancer cases are hereditary. These breast cancers can be associated with poorer health outcomes. Taking a family history can identify women at increased cancer risk. Women at higher than population risk should be referred for assessment. Those at population risk would be cared for by their doctor. However, it is still unclear what psychological impact of proactively screening for breast cancer has on women.
Methods and Materials: Eligible women were invited from 4 general practices. Participants completed family history and psychological outcome questionnaires at baseline, with follow-up outcome questionnaire at 2 weeks. Participants' risk of familial breast cancer was assessed using bespoke computer programme (FaHRAS). Outcomes related to anxiety, emotional response, worry and perceived risk were measured pre- and post-intervention.

Results: Outcome variables were low at baseline, scores in the lower half of their range, specifically state anxiety with median score of 33/80 for the whole cohort. There was no significance change from baseline for 13/18 of outcomes tested. The most significant increases in median anxiety scores were from baseline to follow-up in the higher than population risk group $(\mathrm{p}=.032, r=.18)$. There were significant decreases in median breast cancer worry from baseline to follow-up in population risk group $(\mathrm{p}=.001$, $r=.18)$ and median perceived relative risk $(\mathrm{p}=.009$, $r=.14)$.

Conclusions: This study suggests proactive family history screening does not induce adverse psychological impact in most women assessed. However, anxiety did significantly increase in the higher than population group which requires further exploration.

Grant reference: 205 and 206

B. Dutton: None. N. Qureshi: None. S. Weng: None. E. Robertson: A. Employment (full or part-time); Significant; FaHRAS.

\section{EMP1.23C}

The psychological impact of Rapid BRCA1/2 genetic testing to guide cancer treatment

\section{A. Dearing, S. Limb, K. Kohut, K. Snape, H. Hanson}

St George's Hospital, London, United Kingdom

Introduction: Increasingly, genetic test results are used to influence cancer treatment. To meet this need, the South West Thames Regional Genetics Service developed a "Rapid Access" pathway. Eligible women are consented for BRCA1/2 analysis by their Oncology team at the point of diagnosis, and then contacted by the Clinical Genetics department within two working days to discuss. However, the psychological impact of this pathway is unclear.

Aims: To gain an understanding of the psychological response of women who have genetic testing at the point of diagnosis ("Rapid Patients, RP"), and compare it to women who have genetic testing after their cancer treatment has completed ("Diagnostic Patients, DP").

Methods: RP $(n=98)$ and DP $(n=99)$ who were referred between January 2017 and December 2017 were sent online surveys. The survey was composed of service 
satisfaction items modified from George et al. (2016), the Multidimensional Impact of Cancer Risk Assessment (MICRA) Questionnaire, and open-ended, qualitative questions.

Preliminary Results and Discussion: Twenty-six (13\%) participants have completed the survey to date $(07 / 02 /$ 2018), and data collection is on-going. Although both subgroups report being satisfied with the process and quality of genetic counselling, RPs scored higher on distress and uncertainty subscales on the MICRA. This may reflect their feelings of distress and uncertainty with their on-going cancer treatment and prognosis. Analysis of the complete data set will be presented, identifying significant differences between the two groups. This work may have implications for practice, including providing on-going psychological support to women receiving treatment.

A. Dearing: None. S. Limb: None. K. Kohut: None. K. Snape: None. H. Hanson: None.

\section{EMP1.24D}

Decision-making and experience of tamoxifen as chemoprevention to manage breast cancer risk for young women with a BRCA1/2 mutation

\section{E. Forrest ${ }^{\text {, R. Forbes Shepherd }}{ }^{1}$, M. Young ${ }^{2}$, L. A. Keogh ${ }^{3}$, P. A. James ${ }^{1}$}

${ }^{1}$ Peter MacCallum Cancer Centre, Melbourne, Australia, ${ }^{2}$ The Garvan Institute of Medical Research, Sydney, Australia, ${ }^{3}$ The University of Melbourne, Melbourne, Australia

Introduction: Tamoxifen can be offered to premenopausal women at high risk of breast cancer, and can reduce breast cancer risk by approximately $38 \%$. Tamoxifen is a less invasive option for risk management than risk reducing surgery, yet uptake among young women with BRCAl/2 mutations is low. Evidence about decision-making and adherence to tamoxifen stems largely from clinical trials with older women. This study examines decision-making and experience of tamoxifen by young women with BRCAl/ 2 mutations. Methods: Women aged 18-40 years with BRCA1/2 mutations predominantly from Victoria, Australia, were invited to participate in a qualitative interview exploring their psychosocial needs. A grounded theory approach informed study design; data were analysed iteratively and inductively.

Results: Thirty-eight interviews with women aged 20-40 years with BRCA1/2 mutations were conducted in 2015 . Twenty-one had chosen not to take tamoxifen, six had taken tamoxifen, and the remainder could not recall discussing chemoprevention with their healthcare provider or were too young to commence cancer risk management. Women's tamoxifen-related decisions were frequently influenced by childbearing plans, conversations with their healthcare providers, and cancer risk perception. All the women who had taken tamoxifen experienced transient side effects, yet all bar one continued with the medication. Conclusion: Decision-making about tamoxifen was nuanced and informed by considerations characteristic of young adulthood, especially childbearing. While few participants chose tamoxifen, it is important to offer young women with BRCA1/2 mutations different risk management strategies to enable choices that meet their needs during young adulthood. Funding: National Breast Cancer Foundation (PF-14-009), Australia.

L.E. Forrest: None. R. Forbes Shepherd: None. M. Young: None. L.A. Keogh: None. P.A. James: None.

\section{EMP1.25A}

Evaluation of the eviQ genetic testing guidelines and riskmanagement for people with hereditary cancer syndromes : Survey and audit of Familial Cancer Clinics

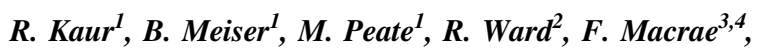 J. Hiller ${ }^{5}$, J. Kirk ${ }^{6}$, A. Trainer ${ }^{7}$, G. Mitchell ${ }^{7,8}$
${ }^{1}$ Prince of Wales Clinical School, Sydney, Australia, ${ }^{2}$ University of Queensland, Brisbane, Australia, ${ }^{3}$ The Royal Melbourne Hospital, Melbourne, Australia, ${ }^{4}$ University of Melbourne, Melbourne, Australia, ${ }^{5}$ Swinburne University of Technology, Hawthorn, Australia, ${ }^{6}$ Crown Princess Mary Cancer Centre at Westmead Hospital, Sydney, Australia, ${ }^{7}$ Peter MacCallum Cancer Centre, Melbourne, Australia, ${ }^{8}$ The Sir Peter MacCallum Department of Medical Oncology, University of Melbourne, Melbourne, Australia

Introduction: Web-based point-of-care cancer genetics guidelines were launched across Australia in 2005 (http://www.eviq.org.au) to support the clinical decisionmaking in patient care. This project aimed to assess adherence to guidelines. Materials and methods: Data collection was performed by trained genetic counsellors at 13 familial cancer clinics across Australia. (i) A file audit was carried out to assess compliance with guidelines regarding selection of patients for an offer of genetic testing for heritable mutations in the BRCAl/2 genes. (ii) From each familial cancer clinic, a random sample of unaffected carriers of BRCA1, BRCA2 and Lynch Syndrome-related mutations were selected. Patients were interviewed to assess their adherence to recommended risk management guidelines and barriers in compliance to guidelines.

Results: (i) A total of 1,053 files were audited. Preliminary data analysis shows a high interrater reliability (k-statistics $=0.89$ ). Compliance of genetic testing uptake after the introduction of guidelines was more than $70 \%$. In more than $95 \%$ of noncompliant cases publicly funded 
genetic testing was used. (ii) $168 B R C A 1,161 B R C A 2$ and 215 Lynch Syndrome-related mutation carriers were interviewed. Twenty-eight percent to $32 \%$ of known mutation carriers for $B R C A 1 / 2$ are in the younger age group and are waiting to complete their families before completing breastcancer related risk-reducing surgery. Other reasons commonly cited for non-adherence were lack of awareness, past bad experience and fear of adverse effects.

Conclusion: This study provides data on the reasons for non-adherence to risk management recommendations amongst patients and thus provides the basis for the development of interventions to address such nonadherence.

R. Kaur: None. B. Meiser: None. M. Peate: None. R. Ward: None. F. Macrae: None. J. Hiller: None. J. Kirk: None. A. Trainer: None. G. Mitchell: None.

\section{EMP1.26B}

I had already made up my mind: a qualitative interview study on Italian women participating in clinical cancer genetic counseling and testing of the BRCA genes

\section{Battistuzzi ${ }^{1}$, M. Franiuk ${ }^{2}$, O. Puricelli ${ }^{3}$, N. Rania ${ }^{4}$,} L. Migliorini ${ }^{5}$, L. Varesco ${ }^{6}$

${ }^{1}$ Dept of Informatics, Bioengineering, Robotics and Systems Engineering - Dept of Internal Medicine and Medical Specialities, Genoa, Italy, ${ }^{2}$ Ospedale Policlinico San Martino Unit of Herditary Cancer, Genoa, Italy, ${ }^{3}$ Ospedale Policlinico San Martino - Unit of Clinical Psychology and Psychotherapy, Genoa, Italy, ${ }^{4}$ University of Genoa - DISFOR - Department of Education Sciences, Genoa, Italy, ${ }^{5}$ Univesity of Genoa DISFOR - Department of Education Sciences, Genoa, Italy, ${ }^{6}$ Ospedale Policlinico San Martino - Unit of Hereditary Cancer, Genoa, Italy

Introduction: As cancer panel testing is becoming routine clinical practice, the need has emerged to develop new models of Cancer Genetic Counseling (CGC). Developing such new models requires that efforts be directed at understanding how best to support patients' decisionmaking in CGC. To date, most of the research that has investigated the experiences of individuals participating in diagnostic BRCA testing has focused on English-speaking or Northern-European populations, and very little is known about patients and families from Southern Europe.

Materials and Methods: We conducted in-depth semistructured interviews with 19 Italian women with no personal history of cancer who had had BRCA testing in a clinical setting.

Results: A number of themes emerged; here we focus on three that are related to decision-making: "I had already made up my mind", "Thinking it through", and "The right thing to do". Analysis of the interviews showed that most of the respondents had decided whether to pursue BRCA testing before their pre-test counseling session; the decision to be tested was often reported to have been quick and straightforward, and the dimension of choice was interwoven with notions of responsibility and moral obligation.

Conclusions: While not generalizable, these findings suggest that a subset of Italian women may arrive at their first CGC consultation having already made a decision about testing. New models of CGC could usefully take into account differences in how women approach the testing process to ensure that informed consent and CGC protect them from the potential risks of testing.

L. Battistuzzi: None. M. Franiuk: None. O. Puricelli: None. N. Rania: None. L. Migliorini: None. L. Varesco: None.

\section{EMP1.27C}

Young cancerpatient perspectives on undertaking whole genome sequencing: A qualitativestudy

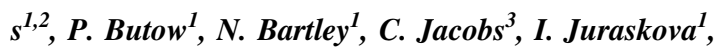
A. Newson ${ }^{2}$, J. Savard ${ }^{2}$, B. Meiser ${ }^{4}$, M. Ballinger ${ }^{5}$, D. Thomas ${ }^{5}$, B. Biesecker ${ }^{6}$

${ }^{1}$ School of Psychology, University of Sydney, Australia, ${ }^{2}$ Sydney Health Ethics, University of Sydney, Australia, ${ }^{3}$ Prince of Wales Clinical School, University of NSW, Australia, ${ }^{4}$ University of NSW, Sydney, Australia, ${ }^{5}$ Garvan Institute of Medical Research, Sydney, Australia, ${ }^{6}$ RTI International, Washington, MD, United States

Introduction: Molecular tumour profiling (MTP), aiming to link molecular targets in tumours to personalised therapies, is entering clinical practice. Little is yet known about the ethical, psychosocial and behavioural implications of MTP. The current longitudinal psychosocial study (PiGeOn) aims to explore understanding, experiences and views regarding MTP for 1,000 patients with an advanced, solid, rare cancer who undergo testing. Baseline qualitative results are reported here.

Materials and Methods: Purposive sampling is used to ensure diversity in cancer types and demographics. PiGeOn participants participate in semi-structured interviews which are analysed by Framework analysis.

Results: Data collection is ongoing. Thus far, participants' motivations include: fear of death, trust in their oncologist, need for control and a sense of 'nothing lost'. Desperation and fear of dying dominate motivation, but participants also value being able to help others in a similar situation, and the chance to benefit other family members. Participants tolerate uncertainty and hope for reduced prognostic uncertainty, but fear loss of hope if no actionable 
result is found. They often lack understanding, but trust the science of MTP and the research process.

Conclusions: Identifying new treatment options overshadows broader and familial implications of genetic testing in this population. The current data will inform future policy and practice on how to effectively engage/support patients in the MTP decision-making process, and how uncertainty should be managed in this complex area.

This study is sponsored by a Grant from the National Health and Medical Research Council Australia.

M. Best: None. P. Butow: None. N. Bartley: None. C. Jacobs: None. I. Juraskova: None. A. Newson: None. J. Savard: None. B. Meiser: None. M. Ballinger: None. D. Thomas: None. B. Biesecker: None.

\section{EMP1.28D}

Genetic counselling outcomes for lifepool research participants notified of HBOC mutations via a letter providing direct access to a telephone genetic counsellor

\section{A. Lewis ${ }^{1}$, M. Young ${ }^{2}$, L. Devereux ${ }^{1}$, P. A. James ${ }^{1}$, A. H. Trainer ${ }^{1}$ \\ ${ }^{1}$ Peter MacCallum Cancer Centre, Melbourne, Australia, ${ }^{2}$ Genome.One Garvan Institute of Medical Research, Darlinghurst, Australia}

Genetic research may produce information that has clinical implications for research participants and their family. Uptake of clinical genetics referral and confirmatory genetic testing for research participants notified via letter of the availability of genetic health information has been poor in several Australian studies (between 8-56\%).

Research studies have identified access to a genetic counsellor as a barrier for some to act on mutation notification information. To overcome this barrier a model was developed that provided research participants notified via letter with direct telephone access to a genetic counsellor (TGC).

This model was used to notify participants found to carry clinically actionable hereditary breast and ovarian cancer (HBOC) mutations in the Australian population based study of healthy women, lifepool.

Thirty five women in this cohort identified to have a HBOC mutation were notified via letter that "information of relevance to their health" had been identified, with the letter containing the direct contact details of a genetic counsellor. Thirty two (91\%) women contacted the genetic counsellor directly after receiving the letter, all of whom accepted referral to a clinical genetics service. Thirty one women $(88 \%)$ underwent confirmatory genetic testing after genetic counselling. TGC provided the women notified opportunity to discuss the letter's content and allowed onward referral to be facilitated.

The inclusion of a telephone genetic counsellor in the notification protocol for lifepool has resulted in a significantly higher uptake of onward referral to clinical genetics services compared to other Australian studies that have relied upon a letter alone.

A. Lewis: None. M. Young: None. L. Devereux: None. P.A. James: None. A.H. Trainer: None.

\section{EMP1.29A}

The psychosocial experiences of Australian adolescents and young adults with Li-Fraumeni Syndrome: a qualitative study R. Forbes Shepherd ${ }^{1,2,3}$, L. A. Keogh ${ }^{4}$, A. Werner-Lin
,
M. B. Delatycki ${ }^{3,6,7}$, L. E. Forrest

${ }^{1}$ Parkville Familial Cancer Centre, Peter MacCallum Cancer Centre, Melbourne, Australia, ${ }^{2}$ Sir Peter MacCallum Department of Oncology, The University of Melbourne, Melbourne, Australia, ${ }^{3}$ Bruce Lefroy Centre for Genetic Health Research, Murdoch Children's Research Institute, Melbourne, Australia, ${ }^{4}$ Melbourne School of Population and Global Health, The University of Melbourne, Melbourne, Australia, ${ }^{5}$ School of Social Policy and Practice, University of Pennsylvania, Philadelphia, PA, United States, ${ }^{6}$ Department of Paediatrics, University of Melbourne, Melbourne, Australia,

${ }^{7}$ Victorian Clinical Genetics Service, Melbourne, Australia

Introduction: Li-Fraumeni Syndrome (LFS), a devastating cancer predisposition caused by germline mutations in TP53, confers considerable multi-organ cancer risks from childhood. Adolescents and young adults (AYAs: aged 1529 years) with LFS experience their transitional life stage of emerging adulthood concurrently with the prospect of intensive life-long surveillance, limited cancer prevention options, cancer diagnoses and treatment, and for some, mortality. The complex and unique psychosocial needs for AYAs with LFS remain critically understudied. This is the first study internationally to explore the psychosocial experiences of AYAs with LFS and make recommendations for genetic counselling practice.

Materials and Methods: Australian AYAs with a TP53 germline mutation were invited to participate in a semistructured interview examining the psychosocial impact of LFS. Data collection and analysis is ongoing, informed by interpretive description and the Family System Genetic Illness model.

Results: Fifteen interviews have been conducted to date (mean age 22). Familial and personal experiences of cancer were highly varied and influenced risk perception and cancer fear. Comprehensive screening was critical in 
buffering LFS-related distress. Despite this, feelings of isolation were common, especially among AYAs with de novo mutations $(\mathrm{n}=3)$, as were feelings of personal burden with managing LFS. AYAs strove for an identity independent of LFS and although it had a limited day-to-day impact, LFS remained a grave concern for most.

Conclusions: AYAs with LFS follow a range of psychosocial trajectories through development and could benefit from tailored longitudinal care from a psychosocial genetically-oriented multidisciplinary team. Funding: Melbourne Research Scholarship, The University of Melbourne.

R. Forbes Shepherd: None. L.A. Keogh: None. A. Werner-Lin: None. M.B. Delatycki: None. L.E. Forrest: None.

\section{EMP1.30B}

Prenatal chromosomal microarray analysis: Exploring views of genetics healthcare professionals on disclosure of susceptibility loci

\section{Macarov', V. Meiner ${ }^{1}$, V. Libman', H. Hochner ${ }^{2}$, S. Shkedi- Rafid $^{1}$}

${ }^{1}$ Department of Genetics and Metabolic Diseases, Hebrew University-Haddasah Medical Center, Jerusalm, Israel, ${ }^{2}$ Braun School of Public Health, Hebrew University-Haddasah Medical Center, Jerusalm, Israel

Introduction: Chromosomal microarray analysis (CMA) in prenatal diagnosis allows for an increased diagnostic yield of pathogenic copy-number variations (CNVs), but also identifies CNVs with incomplete penetrance, associated with increased risk for neurodevelopmental phenotypes (susceptibility loci, SL). Practices regarding prenatal disclosure of SL range from full-disclosure to non-disclosure of low-penetrance SL. There is no consensus as to whether parents should be given a choice regarding which findings to receive. This study explored views of Israeli healthcare professionals (HCPs) on disclosure of SL and the factors influencing these views.

Methods: Semi-structured interviews with twenty Israeli HCPs analyzed using grounded theory methodology.

Results: SL are routinely reported, regardless of penetrance. Full-disclosure is supported by the view that providing this information allows women/parents to make informed choices regarding the pregnancy's future. Nondisclosure is perceived as paternalistic, and as not conforming to Israeli norms, where couples generally seek to avoid any risks for their future child. The minority of participants believe that low-penetrance SL should not be reported, as they cause anxiety and unjustified pregnancy terminations. Nevertheless, they do report SL because of their fear of lawsuits on medical malpractice if a child is subsequently diagnosed with neurodevelopmental problems. Interviewees largely oppose parental-choice in disclosure of genomic information due to its complexity.

Conclusions: Support in reporting low-penetrance SL is either based on perceiving this information as helpful, or on fear of medical practice litigation. Participants largely prioritize their own judgment about disclosure over parental-choice. Funding: Israel NIHP grant 2015/82.

M. Macarov: None. V. Meiner: None. V. Libman: None. H. Hochner: None. S. Shkedi-Rafid: None.

\section{EMP1.31C}

User acceptability of whole exome pre-conception carrier testing for consanguineous couples in Australia

K. K. Barlow-Stewart ${ }^{1}$, S. Josephi-Taylor ${ }^{2,3}$, A. Selvanathan ${ }^{4}$, T. Roscioli ${ }^{2}$, A. Bittles ${ }^{5}$, B. Meiser ${ }^{6}$, L. Worgan $^{7}$, S. Rajagopalan ${ }^{7}$, A. Colley $^{7}$, E. Kirk ${ }^{8,2,3}$

${ }^{1}$ University of Sydney, Sydney, Australia, ${ }^{2}$ Centre for Clinical Genetics, Sydney Children's Hospital, Sydney, Australia, ${ }^{3}$ University of NSW, Sydney, Australia, ${ }^{4}$ Clinical Genetics Services, Liverpool Hospital, Sydney, Australia, ${ }^{5}$ School of Medical and Health Sciences, Edith Cowan University, Perth, Australia, ${ }^{6}$ University of New South Wales, Sydney, Australia, ${ }^{7}$ Clinical Genetics Services, Liverpool Hospital, Sydney, Australia, ${ }^{8}$ Genetics Laboratory, NSW Health Pathology East, Sydney, Australia

Purpose: To explore with consanguineous couples in Australia the acceptability and perceived utility of preconception clinical exome screening for autosomal recessive and X-Linked conditions.

Methods: Semi-structured interviews with 21 consanguineous couples of diverse ethnic and religious backgrounds conducted prior to offering preconception screening. Interviews were thematically analysed using by an inductive approach.

Results: Three major themes were identified: the consanguineous couple; childhood disorders; and perceived utility of genomic screening. 16/21 reported consanguinity as being common within their own community; several reported surprise at learning after immigrating that it was uncommon in Australia. While no stigma was reported, 6/ 21 expressed concerns about its potential and disclosure of their consanguinity in the wider community was limited. 20/ 21 were aware of childhood disorders within their family or community; 13/21 perceived consanguinity as increasing the risk of having affected children. While explanatory beliefs included war as a source of stress, exposure to chemicals released by bombs and unclean water, none expressed disbelief in genetics as a contributor. Nine spoke 
of premarital screening programs routinely conducted in their countries of origin. 14/21 proceeded with genomic testing; however, all supported the availability of genomic preconception screening. If found to be carriers of a severe childhood disorder, 13/21 would test a pregnancy; twelve would consider termination of pregnancy or preimplantation genetic diagnosis. A majority of couples would communicate a potential at risk status to family members.

Conclusion: Those who chose to have testing would utilise the results with the goal of preventing childhood disorders.

K.K. Barlow-Stewart: None. S. Josephi-Taylor: None. A. Selvanathan: None. T. Roscioli: None. A. Bittles: None. B. Meiser: None. L. Worgan: None. S. Rajagopalan: None. A. Colley: None. E. Kirk: None.

\section{EMP1.32D}

Split opinions: A qualitative study exploring Genetic Counsellors' attitudes and experiences of direct-toconsumer genetic testing

E. James ${ }^{1}$, C. Lewis ${ }^{2}$, N. Taverner ${ }^{1}$, M. McAllister ${ }^{1}$

${ }^{1}$ Institute of Medical Genetics, Cardiff University School of Medicine, Cardiff, United Kingdom, ${ }^{2}$ Great Ormond Street Hospital and UCL Institute of Child Health, London, United Kingdom

Aim: The market for Direct-to-Consumer Genetic Testing (DTC-GT) is rapidly growing, with increased uptake, and lack of post-test support leading many patients to seek help from their local health services. The aim of this study was to explore the implications of DTC-GT for clinical genetics practice, and consider what shapes genetic health care professionals' (HCPs) attitudes towards DTC-GT.

Method: This qualitative study used semi-structured interviews to collect data from 12 Genetic Counsellors with experience of seeing patients regarding DTC-GT. A thematic analysis was then conducted on the resulting transcripts.

Results: The participants expressed divided opinions, with some "rejecting" DTC-GT and others "cautiously accepting" the technology. These split opinions resulted in an exploration of a range of themes considering both advantages and disadvantages of this technology. Core themes highlighted negative implications of DTC-GT including information imbalance, patient abandonment, negative psychosocial impact and strain on health care services. In a more positive light, participants also discussed the empowerment provided by these tests, and a hope for the future. These themes lead to the formation of a theoretical framework considering how participants apply their own justified paternalism to DTC-GT, weighing the pros and cons of the testing, regardless of their personal experiences.

Conclusion: This study provides evidence for how genetic counsellors' opinions differ substantially regarding DTC-GT. These divergent viewpoints underscore the importance of a coordinated approach in tackling the issues surrounding DTC-GT and its impact on clinical genetics services, with many participants calling for consistency and regulation.

E. James: None. C. Lewis: None. N. Taverner: None. M. McAllister: None.

\section{EMP1.33A}

Case study: Ethical issues around sharing of information when a baby is born with a genetic condition following IVF with sperm donation

\section{Kosicka-Slawinska}

North West Thames Regional Genetics Service, Harrow, United Kingdom

We present a genetic counselling case where a baby was diagnosed with, and died of GM1-gangliosidosis (lipid storage disorder) following IVF with sperm donation.

We describe the communication which occurred between the genetic counsellor and couple, genetic counsellor and fertility centre, and genetic counsellor and sperm donor.

The case raised several issues regarding confidentiality of both the couple and the donor and the role of the genetic counsellor in such a situation. The Human Fertilisation and Embryology Authority (UK) have clear guidelines on what the fertility centre should do in such an event so that other couples who have conceived using the same donor sperm can be alerted.

In this case the donor wondered whether to inform his own (adult) daughter about the genetic diagnosis because the carrier frequency of GM1-gangliosidosis is low and carrier testing is not routinely offered to unrelated partners of carriers. The donor worried it would raise anxiety for his daughter and bring into focus his decision to be a sperm donor.

The donor's decision to have carrier testing in this case would impact on the couple's ability to go ahead with PGD in a future pregnancy as they had stored frozen embryos. We comment on the role of the genetic counsellor in these discussions.

We draw a comparison with another case, where a couple were referred to Genetics because they had been informed that their sperm donor had fathered a baby with PTS deficiency.

M. Kosicka-Slawinska: None. 


\section{EMP1.34B}

Professional's opinions of genetic information and testing in the context of adoption

\author{
N. Jackson ${ }^{1}$, K. Burke ${ }^{2}$, A. Clarke $^{2}$ \\ ${ }^{1}$ University Hospitals Bristol, Bristol, United Kingdom, \\ ${ }^{2}$ Cardiff University, Cardiff, United Kingdom
}

Introduction: Looked-after children (LAC) with disabilities are some of those who wait longest for permanency. For many of these children, the assessment of their health and developmental needs will involve family history information and genetic testing. This study explored professionals' opinions and experiences concerning family medical information and genetic/genomic testing in the adoption process in Wales, considering its utility and the attitudes surrounding it.

Method: Semi-structured qualitative interviews were conducted with 6 social workers and 7 medical advisors. The data was transcribed and thematically analysed.

Results: Professionals described challenges to collation of family history of the LAC, in particular a lack of understanding of the relevance of genetic information. Medical advisors described altering the testing 'threshold' for children awaiting adoption to try and establish a diagnosis and expressed concern about the possible uncertainty of information arising from such testing. This uncertainty was expressed by all professionals, especially towards the impact of genetic information and how it is understood and handled by professionals and prospective parents on a child's placement. In response to this there was a call from professionals for improved training and for increased networking between genetics services and those working with LAC.

Conclusions: There are particular challenges around collecting and sharing genetic information and professionals lack confidence in their knowledge and the protocols involved. As new genetic technologies increase the scope, rather than the nature, of these challenges, a programme of genetic education could be implemented for social workers and local doctors working in adoption.

N. Jackson: None. K. Burke: None. A. Clarke: None.

\section{EMP1.35C}

Genomic counselling: what are we doing and what do we think we should be doing? - A professional status survey of genetic counsellors in Australasia

\author{
A. Nisselle ${ }^{1,2,3}$, I. Macciocca ${ }^{2,4,5}$, K. Dunlop ${ }^{1,6}$, S. Metcalfe ${ }^{1,2,3}$, \\ C. Gaff ${ }^{1,2,3}$, Australian Genomics Health Alliance Genomic \\ Workforce, Education \& Ethics Working Group
}

${ }^{1}$ Australian Genomic Health Alliance, Parkville, Australia,

${ }^{2}$ Murdoch Children's Research Institute, Parkville, Australia,

${ }^{3}$ The University of Melbourne, Parkville, Australia,

${ }^{4}$ Australasian Society of Genetic Counsellors, Sydney, Australia, ${ }^{5}$ Victorian Clinical Genetics Service, Parkville, Australia, ${ }^{6}$ Centre for Genetics Education, NSW Health, St Leonards, Australia.

Introduction: Work practices of genetic counsellors (GCs) are changing rapidly in response to the introduction of genomics in healthcare and 'direct-to-consumer' tests. A professional census by the Australasian Society of Genetic Counsellors (ASGC) and Australian Genomics Health Alliance, provides insights into the current status of the profession and preferred future genomics practice.

Materials and Methods: Online survey developed, piloted and deployed February-April 2017 to ASGC members and Australian GC training program graduates. Data analysed using descriptive and inferential statistics and content analysis.

Results: Surveys were completed by 271 respondents with GC qualifications (56.3\% response). $96.3 \%$ were female; $65.9 \%$ worked in clinical roles, $10.7 \%$ in laboratory/research, $4.6 \%$ administration/education and $17.6 \%$ other. Of those working clinically $(\mathrm{n}=172), 41.3 \%$ were certified and $55.8 \%$ becoming certified. $74.7 \%$ worked for publicly-funded genetics/pathology services, $22.5 \%$ had multiple jobs and $79.4 \%$ reported unpaid overtime. $74.2 \%$ of all GCs recently attended genomics education and many clinical GCs already incorporated genomics into practice assisting patients with a DTC test $(64.4 \%)$ or regularly completing a task associated with chromosomal microarray $(62.5 \%)$, non-invasive prenatal testing $(51.4 \%)$ or whole genome/exome sequencing (WE/GS; 48.6\%). All GCs wanted to become more involved in WE/GS in the future, including creating gene lists $(40.5 \%)$, providing phenotypic information $(55.2 \%)$, variant curation $(44.8 \%)$ and literature searches when reporting results $(59.9 \%)$.

Conclusions: These data provide critical insight for workforce planning into the education, training, accreditation and work practices of genetic counsellors, and a baseline for longitudinal comparisons as genomics becomes part of routine health care.

Grant reference: GNT1113531

A. Nisselle: None. I. Macciocca: None. K. Dunlop: None. S. Metcalfe: None. C. Gaff: None.

\section{EMP1.36D}

The experiences, motivations and expectations of participants in a research project implementing genomics as a paediatric diagnostic service in Wales 
G. A. Pinto Juma ${ }^{1}$, S. Doheny ${ }^{1}$, I. Tully ${ }^{1,2,3}$, A. J. Clarke ${ }^{1,2}$, R. Butler ${ }^{2}$

${ }^{1}$ Institute of Medical Genetics, School of Medicine, Cardiff University, Cardiff, United Kingdom, ${ }^{2}$ All Wales Medical Genetics Service, University Hospital of Wales, Cardiff, United Kingdom, ${ }^{3}$ Neuroscience and Mental Health Research Institute (NMHRI), Cardiff University, Cardiff, United Kingdom

Introduction: The project, 'Studying the Implemention of GeNomics in wALes (SIGNAL)' proposed to investigate the clinical, economic and psychosocial impacts of genomic testing in the diagnosis of paediatric genetic disease. The present nested sub-project aimed to understand the experiences, motivations and expectations of the participants in the SIGNAL project and their views regarding the use of genomics as part of their family's healthcare.

Methods: Families with children affected by developmental delay were recruited to SIGNAL and underwent exome or whole genome sequencing. Data was collected using a questionnaire survey of participants and interviews were conducted with a subset of participants.

Results: This study demonstrated that a central theme of 'trust, hope and progress' was implicit in the participants' experiences, and structured their motivations and expectations. Participation in genomic research was seen as promoting self and social empowerment as the results could reduce isolation and contribute to the overall good. The project uncovered issues with securing informed consent and participants did not feel the need to 'understand' the genomic test. This lack of understanding affected how some participants managed the wait for their results.

Conclusions: The findings demonstrate the willingness, even eagerness, of many families to access genomic investigations. However, they also emphasise the contested nature of what constitutes (informed) 'consent'. In addition, they show that most research participants wish to remain updated on progress throughout the project. The role of genetic counsellors in the negotiation of consent remains to be defined, especially as genomics moves into mainstream medicine.

G.A. Pinto Juma: None. S. Doheny: None. I. Tully: None. A.J. Clarke: None. R. Butler: None.

\section{EMP1.37A}

Implementation of a laboratory-based genetic counsellor in an Australian tertiary paediatric hospital

\author{
Y. Smagarinsky ${ }^{1,2,3}$, I. Macciocca ${ }^{2,3}$, J. Phillips ${ }^{1}$, H. Savoia ${ }^{1}$, \\ S. White ${ }^{2,3}$, L. Curnow ${ }^{1,2,3}$
}

${ }^{1}$ The Royal Children's Hospital, Melbourne, Australia, ${ }^{2}$ Victorian Clinical Genetics Services, Melbourne, Australia,

${ }^{3}$ Murdoch Children's Research Institute, Melbourne, Australia

The Royal Children's Hospital (RCH) in Melbourne, Australia has seen a significant increase in genetic test requests and associated expenditure within the last three years. Prompted by this, two laboratory-based genetic counsellors (LBGCs) employed by the Victorian Clinical Genetics Services (clinical genetics service of $\mathrm{RCH}$ ) have been appointed to a three-year full-time job-sharing role. The overall aim of this appointment is to facilitate a system that ensures access to high-quality, cost-effective, clinically indicated and accurate genetic and genomic testing. The utility of LBGCs has been demonstrated in the USA, but this is the first formal role in Australia to our knowledge. Since the role commenced in 2017, a major responsibility has been overseeing the test request review process which assesses tests according to their clinical utility. Using their skill base, LBGCs are the interface between the referring clinicians and the laboratory, ensuring appropriate clinical information is provided and adequate patient consent obtained. In consultation with senior medical specialists within $\mathrm{RCH}$, assistance is provided to determine the most appropriate test. LBGCs assist in sourcing accredited laboratories with clinically acceptable turnaround times, which enables clinicians to make crucial informed decisions regarding patient care. The LBGCs will also develop streamlined processes for genetic/genomic test ordering and consent, including decision support and just-in-time education of medical specialists. Cases arising from the test request review process demonstrate the value of LBGCs within an Australian health policy context, in turn strengthening a future workplace-model for LBGCs.

The LBGC role is supported by a RCH Foundation grant.

Y. Smagarinsky: None. I. Macciocca: None. J. Phillips: None. H. Savoia: None. S. White: None. L. Curnow: None.

\section{EMP1.38B}

General practitioner views towards providing personal genomic risk information to the general population

\author{
A. K. Smit ${ }^{1,2,3}$, A. J. Newson ${ }^{2}$, L. Keogh ${ }^{4}$, M. Best ${ }^{2,5}$, K. Voon Sen

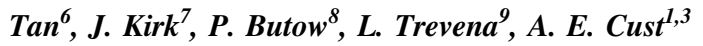 \\ ${ }^{1}$ Cancer Epidemiology and Prevention Research, Sydney \\ School of Public Health, The University of Sydney, Sydney, \\ Australia, ${ }^{2}$ Sydney Health Ethics, Sydney School of Public \\ Health, The University of Sydney, Sydney, Australia, \\ ${ }^{3}$ Melanoma Institute Australia (MIA), The University of \\ Sydney, Sydney, Australia, ${ }^{4}$ Melbourne School of Population \\ and Global Health, The University of Melbourne, Melbourne,
}


Australia, ${ }^{5}$ Psycho-Oncology Co-operative Research Group (PoCoG), The University of Sydney, Sydney, Australia, ${ }^{6}$ The University of Sydney, Sydney, Australia, ${ }^{7}$ Westmead Clinical School and Westmead Institute for Medical Research, Sydney Medical School, The University of Sydney, Sydney, Australia, ${ }^{8}$ Centre for Medical Psychology and Evidence-based Decisionmaking, School of Psychology, The University of Sydney, Sydney, Australia, ${ }^{9}$ Sydney School of Public Health, The University of Sydney, Sydney, Australia

Introduction: Exploring general practitioner (GP; primary care physician) views about providing genomic risk information for common, complex diseases will assist with developing professional educational resources and the potential implementation of this testing in future clinical practice.

Materials and methods: We conducted semi-structured interviews with 22 GPs in New South Wales, Australia. We explored their experiences in managing patients' genomic risk information in current practice and views on potentially providing this information on a population scale. Interviews were transcribed and analysed using qualitative analysis.

Results: GPs described genomic risk information as part of 'preventive healthcare', however, their limited confidence in communicating about genomic risk and concern that research evidence is still preliminary restricted perceptions of the usefulness of this information in current disease prevention. GPs believed that communicating genomic risk will inevitably become part of clinical practice, especially because increasing public awareness is already increasing pressure on clinicians to discuss genomic risk. GPs felt that patients should be able to decide whether they receive genomic risk information. Perceived benefits of delivering this information depended on whether it was likely to change patient management or behaviours. Concerns included potential adverse psychological responses and insurance discrimination. Resources required for the future include disease risk calculators that incorporate genomic and traditional risk factors, succinct evidence-based resources and patient-appropriate educational information.

Conclusions: GPs believe that communicating genomic risk will become part of clinical practice, but they are unprepared and concerned about potential psychosocial and ethical implications. GPs require practical, evidence-based resources and patient-appropriate educational information.

A.K. Smit: None. A.J. Newson: None. L. Keogh: None. M. Best: None. K. Voon Sen Tan: None. J. Kirk: None. P. Butow: None. L. Trevena: None. A.E. Cust: None.

\section{EMP1.39C}

Women victims of intimate partner violence: psychological aspects and future epigenetic perspectives

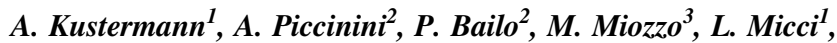
G. Barbara ${ }^{1}$, E. Battaglioli ${ }^{4}$, C. Farè ${ }^{3}$, L. Cirella ${ }^{1}, A$. Pitidis $^{5}$, P. Cremonesi $^{6}$, P. Schinco ${ }^{7}$, T. Emanuele $^{8}$, D. $_{\text {Morero }}{ }^{8}$, S. Gaudi ${ }^{5}$

${ }^{1}$ Department of Women's and Children's Health and Service for Sexual and Domestic Violence (SVSeD), Fondazione IRCCS Ca' Granda Ospedale Maggiore Policlinico, Milan, Italy, ${ }^{2}$ Forensic Genetics Laboratory. Department of Biomedical Sciences for Health - University of Milan, Milan, Italy, ${ }^{3}$ UOS coordinamento laboratori di ricerca, IRCCS Ca' Granda Ospedale Maggiore Policlinico, University of Milan, Milan, Italy, ${ }^{4}$ IDept. Medical Biotechnology and Translational Medicine University of Milan, Milan, Italy, ${ }^{5}$ Italian National Institute of Health, Rome, Italy, ${ }^{6}$ Medicina e chirurgia d'accettazione e d'urgenza - Emergency Department - Galliera Hospital, Genoa, Italy, ${ }^{7}$ General Hospital University of Turin, Turin, Italy, ${ }^{8}$ Maria Vittoria and S. Giovanni Bosco General Hospitals, Turin, Italy

Introduction: Violence against women is a relevant health and social problem, frequently associated with post traumatic stress disorder (PTSD), with negative repercussions on the overall quality of life. The project aim is to evaluate the psycological evolution in parallel with the epigenetic signature in battered women during their psycotherapy.

Materials and Methods: Psychological therapy evaluation will be held by analysing a cohort of 200 women who experienced violence and 400 matched controls, followed for 18 months with medical and psychological appointments. Abbreviated PCL-C Post-Traumatic Checklist 6item Civilian Version and CESD-R Scale for the presence of depressive symtoms will be administrated. A panel of $\mathrm{CpG}$ islands regulating, by methylation, genes involved in the PTSD response will be studied using Mass array spectometry.

Results: In the first six months of multi-centric study 42 women were enrolled as cases, selected by severity and continuity of violence criteria, and 25 as controls. In cases the prevalence of PTSD (score $>=14$ ) was $67.5 \%$ (IC 95.0\%: $53.0 \%$-- 82.0\%) compared to $12.0 \%$ (IC 95.0\%: $0.7 \%-24.7 \%$ ) among controls. Among the first 8 patients that completed first 6 months of follow-up 71.4\% (IC 95.0\%: $38.0 \%$ - 100.0\%) showed an amelioration or remained stable as to PTSD score.

Conclusions: Great contribution to personalised and precision medicine will be given by the epigenetic study in association with the relevant questionnaire. By knowing the mechanisms responsible for resilience, we can derive clues about the best treatment for PTSD and the best public policy.

A. Kustermann: None. A. Piccinini: None. P. Bailo: None. M. Miozzo: None. L. Micci: None. G. Barbara: 
None. E. Battaglioli: None. C. Farè: None. L. Cirella: None. A. Pitidis: None. P. Cremonesi: None. P. Schinco: None. T. Emanuele: None. D. Morero: None. S. Gaudi: None.

\section{EMP1.40D}

A novel approach for offering additional findings to patients - separating the decision from diagnostic testing

E. L. Lynch ${ }^{1,2,3}$, M. Martyn ${ }^{1,3,4}$, A. Kanga-Parabia ${ }^{1,3,4}$, P. James ${ }^{5,6}$, S. Lunke ${ }^{2,3}$, I. Macciocca ${ }^{2,3}$, M. Wallis ${ }^{4,7}$, M. F. Hunter $^{8,9}$, A. Trainer ${ }^{5,6}$, J. Halliday ${ }^{3,4}$, N. Brown ${ }^{2,7}$, L. Keogh ${ }^{4}$, J. Wale ${ }^{1}$, I. Winship ${ }^{4,5}$, Melbourne Genomics Health Alliance, C. Gaff ${ }^{1,4}$

${ }^{1}$ Melbourne Genomics Health Alliance, Melbourne, Australia, ${ }^{2}$ Victorian Clinical Genetics Service, Melbourne, Australia, ${ }^{3}$ Murdoch Children's Research Institute, Melbourne, Australia, ${ }^{4}$ The University of Melbourne, Melbourne, Australia, ${ }^{5}$ The Royal Melbourne Hospital, Melbourne, Australia, ${ }^{6}$ Peter MacCallum Cancer Institute, Melbourne, Australia, ${ }^{7}$ Austin Health, Melbourne, Australia, ${ }^{8}$ Monash Health, Melbourne, Australia, ${ }^{9}$ Monash University, Melbourne, Australia

Genomic sequencing has the potential to reveal information unrelated to diagnostic indications for testing, including the presence of variants that may predict future health risks. Several studies are generating evidence regarding uptake and management of additional findings (AFs) in clinical settings, with consent for AFs at the time of diagnostic testing. We evaluate a novel model, offering reanalysis for AFs to adult patients after they have received genomic sequencing results for their clinical indication. Adults who received diagnostic exome sequencing results are later recontacted to offer reanalysis of their stored data for AFs. Those interested receive decision support materials and genetic counselling before deciding whether to consent and after receiving results of reanalysis. Evaluation focusses on aspects impacting on how AFs are provided, including patient decision-making, uptake, clinical and laboratory service impact, psychosocial impact and views on future service delivery. Evaluation of health impact will not be feasible due to the recruitment target of 100 participants undergoing reanalysis. To date, 20 patients have been approached, with 10 opting to attend a genetic counselling appointment. Three actively opted-out, citing logistical reasons or preference to address health problems as they arise. Of those approached to date, $86 \%$ had earlier expressed hypothetical interest in receiving AFs. An additional 80 patients will be approached by June 2018. Results from this novel model will provide Australian data to complement international studies and will enable comparison with studies in healthy adults and paediatric cohorts.
E.L. Lynch: None. M. Martyn: None. A. KangaParabia: None. P. James: None. S. Lunke: None. I. Macciocca: None. M. Wallis: None. M.F. Hunter: None. A. Trainer: None. J. Halliday: None. N. Brown: None. L. Keogh: None. J. Wale: None. I. Winship: None. C. Gaff: None.

\section{EMP1.41A}

The issue of the fate of a foetus with a mutation after a prenatal diagnosis of spinocerebellar ataxia type $I$ in comparison with myotonic dystrophy in the Yakutia (Sakha Republic)

\author{
S. K. Kononova ${ }^{1,2}$, O. G. Sidorova ${ }^{l}$, M. A. Varlamova ${ }^{l}$, \\ K. A. Kurtanov ${ }^{1}$, F. A. Platonov ${ }^{2}$, V. L. Izhevskaya ${ }^{3}$, \\ E. K. Khusnutdinova ${ }^{4}$, S. A. Fedorova ${ }^{2}$ \\ ${ }^{1}$ Yakut Scientific Centre of Complex Medical Problems, \\ Yakutsk, Russian Federation, ${ }^{2}$ M.K. Ammosov North-Eastern \\ Federal University, Yakutsk, Russian Federation, ${ }^{3}$ Medical \\ Genetics Research Center RAMS, Moscow, Russian \\ Federation, ${ }^{4}$ Institute of Biochemistry and Genetics, Ufa, \\ Russian Federation
}

We discuss one of the issues of prenatal diagnostics - the bioethical dilemma of the fate of a foetus with a mutation. For this issue, we compare two of the most widespread hereditary diseases in the Sakha Republic (Yakutia): spinocerebellar ataxia type 1 (SCA1) and myotonic dystrophy (DM). We believe that prior to the onset of the disease, the individuals-carriers of SCAl gene mutation cannot be called diseased, as they are completely healthy in their physical and intellectual development. Among the carriers of SCAI gene mutation there were and there are many well-known sportsmen, political and public figures, scientists etc. Let us think for a moment: by suggesting a termination of pregnancy to parents of carriers of SCA1 disease, we are, in essence, suggesting to get rid of a productive member of society. Unlike SCA1, DM has a type that causes birth defects. The phenomenon of DM anticipation depends heavily on inheritance from diseased mothers and increases the risk of birth of an almost completely unviable baby with a severe congenital DM. An ethical dilemma arises: "Should we classify spinocerebellar ataxia type 1 as a 'less serious' genetic disease for prenatal diagnostics, and myotonic dystrophy as a more serious disease for prenatal diagnostics?" This is a very complex issue and requires discussion not only among the specialists, but also lawyers, psychologists and the general population of the republic. The study was supported by Ministry of Education and Science of Russian Federation \#6.1766.2017, FASO (BRK_0556-2017-0003). 
S.K. Kononova: None. O.G. Sidorova: None. M.A. Varlamova: None. K.A. Kurtanov: None. F.A. Platonov: None. V.L. Izhevskaya: None. E.K. Khusnutdinova: None. S.A. Fedorova: None.

\section{EMP1.42B}

The Cardiogenetics consultation : an inventorisation of the Liège population

\section{S. Bertoli, L. Van Casteren, S. Bulk, V. Bours}

CHU Liège, Liège, Belgium

Introduction: Hereditary cardiac problems are a common reason for clinical genetics consultation. Due to the familial implications, a workup is recommended in order to offer screening to at-risk family members. The purpose of this study is to inventory our cardiogenetics patient population.

Method: We performed a retrospective observational study of the cardiogenetics consultation patients from the CHU Liège (July 2015 - December 2017). Each index patient with a presumed hereditary cardiac problem received a clinical workup. We performed descriptive statistical analyses. Genetic analysis was performed for each index case.

Results: We have seen 55 patients for an hereditary cardiac condition in our cardiogenetics consultation : 19 women and 36 men (mean age $49.1( \pm 15.7)$ years). 23 patients were referred for HCM, 16 patients for DCM and 16 patients for a cardiac arrhythmia, including Brugada syndrome, QT long syndrome and ARVC/D. A causative mutation was found in 5 patients with $\mathrm{HCM}(\mathrm{n}=18$; $27.8 \%)$, in 3 patients with DCM $(\mathrm{n}=12 ; 25 \%)$ and in 4 patients with cardiac arythmia $(\mathrm{n}=10 ; 40 \%)$. A variant of unknown significance (VUS) was found in 8 patients with HCM $(\mathrm{n}=18 ; 44.4 \%)$, in 1 patient with DCM $(\mathrm{n}=12$; $8.3 \%)$ and in 1 patient with cardiac arythmia $(\mathrm{n}=10 ; 10 \%)$.

Conclusion: Hereditary cardiac problems are not rare. These conditions are frequently autosomal dominant. Therefore, a multidisciplinary cardiogenetics consultation including analysis of first degree at-risk family members fulfills a need in the clinic. In our population, in $30.8 \%$ of index patient, a pathogenic mutation was identified.

S. Bertoli: None. L. Van Casteren: None. S. Bulk: None. V. Bours: None.

\section{EMP1.44D}

Genetic counselling and the implications of predictive testing for individuals at risk of $\mathrm{C9orf72}$ gene expansions

\section{J. A. Bailey, N. Lahiri, M. McEntagart}

Southwest Thames Clinical Genetics Service, St George's Hospital, London, United Kingdom

In September 2011, a mutation within the C9orf72 gene was found, which is known to cause almost $40 \%$ cases of inherited Motor Neurone Disease (MND). Since then, it has been possible to offer predictive testing to unaffected family members.

C9orf72 gene expansions are associated with a reduced penetrance, which makes predictive testing complex. Research suggests that only approximately $50 \%$ of individuals with the gene expansion will go on to develop either MND or FTD before the age of 70 . There is currently no treatment or medication to stop the progression of these conditions.

When considering predictive testing for these individuals, we follow the recommended guidelines for Huntington's disease (HD) predictive testing. However, unlike HD testing which would, in most cases, provide some certainty whether or not the disease would manifest, individuals at risk of a C9orf72 expansion are making a decision to be tested for a condition which may or may not manifest in the future. From experience, this makes the decision making complex, and we have concerns about how individuals may cope with this uncertainty post result.

We present a review of the current literature regarding genetic counselling for individuals at risk of C9orf72 expansions, and present findings of a pilot study, which has been carried out to establish the psychological outcomes and experience individuals have when going through testing. This preliminary work will help inform a further wider UK study, to help improve genetic counselling outcomes in individuals at risk of $\mathrm{C} 9$ orf 72 gene expansions.

J.A. Bailey: None. N. Lahiri: None. $M$. McEntagart: None.

\section{EMP1.45A}

Decision-making for additional genomic findings in parents of newborns with congenital deafness

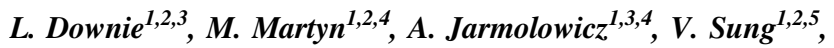
A. Kanga-Parabia ${ }^{1,2,4}$, J. Halliday ${ }^{1,2}$, E. Lynch ${ }^{3,4}$,

Z. Poulakis ${ }^{1,2,5}$, Y. Prawer, ${ }^{4,6}$-. Melbourne Genomics Health Alliance $^{4}$, C. Gaff ${ }^{2,4}$, D. Amor ${ }^{1,2}$

${ }^{1}$ Murdoch Children's Research Institute, Melbourne, Australia, ${ }^{2}$ University of Melbourne, Melbourne, Australia, ${ }^{3}$ Victorian Clinical Genetics Services, Melbourne, Australia, ${ }^{4}$ Melbourne Genomics Health Alliance, Melbourne, Australia, ${ }^{5}$ Royal Children's Hospital, Melbourne, Australia, ${ }^{6}$ Monash Health, Melbourne, Australia 
Genomic sequencing technologies are increasingly available to paediatric patients for diagnostic testing. Research indicates parents are interested in receiving additional findings, when this offer is presented in a hypothetical scenario. However, few studies have explored the decisions parents actually make about additional findings alongside diagnostic testing. To address this, we offered diagnostic genomic testing to eligible infants identified with congenital deafness through a population screening program, 20162017. Consenting parents were given the options of receiving additional findings for treatable or treatable and untreatable childhood onset conditions. They were provided with a decision-aid and pre-test genetic counselling. Parent surveys were sent after consent and results appointments. Evaluation focus includes parent decision-making, uptake of additional findings, psychosocial impact and experience of counselling. To date, 129 parents have been approached: $79(61 \%)$ consented to participation, 26 actively declined, 1 lost to follow-up and 23 are still deciding. $65 \%$ of participants consented to receive additional findings (treatable: $25 /$ 79; treatable and untreatable: 26/79). Participants who requested additional findings hope for information regarding their child's health and wellbeing - for future planning $(28 / 28 ; 100 \%)$ and knowing what to expect in their child's future $(26 / 28 ; 93 \%)$. Most participants $(44 / 46$; 96\%) felt they received enough information about options prior to consent. 93\% (42/45) of respondents agreed they had weighed up pros and cons, selecting the best option for them. More than two-thirds of survey respondents displayed no decisional conflict. This study will inform clinical practise and policy for offering additional findings alongside diagnostic genomic sequencing in paediatric patients.

L. Downie: None. M. Martyn: None. A. Jarmolowicz: None. V. Sung: None. A. Kanga-Parabia: None. J. Halliday: None. E. Lynch: None. Z. Poulakis: None. Y. Prawer: None. -. Melbourne Genomics Health Alliance: None. C. Gaff: None. D. Amor: None.

\section{EMP1.46B}

What are the counselling issues that arise when offering diagnostic whole exome sequencing with targeted analysis for dilated cardiomyopathy in an adult population?

\section{G. Valente ${ }^{1,2}$, J. Ramchand ${ }^{2}$, E. Creed ${ }^{1,3}$, K. West ${ }^{1,3}$, Y. Prawer ${ }^{1,4}$, J. Taylor ${ }^{3}$, M. Bogwitz ${ }^{3}$, I. Macciocca ${ }^{5}$, E. Lynch ${ }^{1}$, M. Wallis ${ }^{2}$, P. James ${ }^{3}$, Melbourne Genomics Health Alliance, C. Gaff}

${ }^{I}$ Melbourne Genomics Health Alliance, Parkville, Australia, ${ }^{2}$ Austin Health, Heidelberg, Australia, ${ }^{3}$ Melbourne Health, Parkville, Australia, ${ }^{4}$ Monash Health, Clayton, Australia, ${ }^{5}$ Victorian Clinical Genetics Services, Parkville, Australia
Introduction: Use of whole exome sequencing (WES) in patients with idiopathic dilated cardiomyopathy (DCM) is expected to increase the molecular diagnostic yield compared to a gene-panel approach. In addition to making cascade testing available for relatives, a molecular diagnosis can impact management of patients if a pathogenic variant is identified in certain genes. WES for patients diagnosed with DCM has only recently been offered in Victoria, Australia. Therefore, it is important to examine the specific counselling issues that arise.

Materials and Methods: Individuals attending an adult genetics clinic between April 2016 and August 2017 were offered WES if they had idiopathic DCM, either diagnosed under the age of 40 , or with a known family history of DCM and/or sudden cardiac death. Participants received genetic counselling prior to consenting to WES and at results. Genetic counsellors prospectively recorded counselling issues that arose during each consultation, which were analysed to identify recurring themes.

Results: Eighty-one eligible adult participants consented to WES. Most participants were male (58.5\%), and the mean age at consent was 47.72 years. The mean length of genetic counselling was 53 minutes for initial appointments and 29 minutes for results appointments. Counselling issues included managing expectations, implications for relatives, insurance and privacy concerns and uncertain family history.

Conclusions: Patients with DCM who undertake WES raise similar counselling issues compared to patients undertaking WES for other reasons. As the use of genomic sequencing increases in DCM, our findings highlight the vital role genetic counsellors play for affected individuals and their families.

G. Valente: None. J. Ramchand: None. E. Creed: None. K. West: None. Y. Prawer: None. J. Taylor: None. M. Bogwitz: None. I. Macciocca: None. E. Lynch: None. M. Wallis: None. P. James: None. C. Gaff: None.

\section{EMP1.47C}

Workshops to improve mainstreaming for BRCA analysis in the West of Scotland

\section{M. Watt, N. Bradshaw, S. Gibson, J. Gorrie, M. Longmuir,} L. Snadden, R. Davidson

West of Scotland Regional Genetics Service, Glasgow, United Kingdom

Workshops were held for colleagues out with genetics to improve the patient care pathway for younger women affected breast cancer with oestrogen receptor negative, progesterone receptor negative and HER2 receptor negative 
pathology and women affected by high grade serous ovarian cancer.

The workshops aimed to increase the knowledge and understanding of BRCA mutation analysis and to provide a practical guide to obtaining blood samples for molecular genetics. By obtaining samples for analysis sooner BRCA results would be available for more timely discussions on medical and surgical management.

47 colleagues from various specialties across the West of Scotland including breast screening, surgery and oncology attended the sessions which were interactive.

Participants were given evaluation forms at the beginning of each workshop to determine their knowledge in three areas then at the end of the workshop colleagues were asked to re-evaluate their knowledge again, similarly colleagues provided feedback on the workshops.

After the workshops comparing the first quarter of 2016 to the first quarter of 2017 samples for BRCA mainstreaming increased from 37 to 75 .

Results:

Q1 How much do you know about how to identify individuals eligible for BRCA testing in the NHS?

Nothing Very little Little Fair understanding Good

\begin{tabular}{lllll}
\hline Start $1330 \mathrm{hrs}$ & 9 & 16 & 6 & 6 \\
End $1630 \mathrm{hrs}$ & & & 30 & 17 \\
\hline
\end{tabular}

Q2 How would you rate your ability to discuss BRCA mutation analysis in the NHS?

\begin{tabular}{|c|c|c|c|c|c|}
\hline & Poor & Not very good & Okay & Good & Very good \\
\hline Start 13 30hrs & & 32 & 8 & 7 & \\
\hline End $1630 \mathrm{hrs}$ & & & & 35 & 12 \\
\hline
\end{tabular}

Q3.How would you rate your understanding about the practical aspects of obtaining a blood sample for molecular genetics?

\begin{tabular}{|c|c|c|c|c|c|}
\hline & Poor & Not very good & Okay & Good & Very good \\
\hline Start 13 30hrs & & 32 & 8 & 7 & \\
\hline End $1630 \mathrm{hrs}$ & & & & 35 & 12 \\
\hline
\end{tabular}

C.M. Watt: None. N. Bradshaw: None. S. Gibson: None. J. Gorrie: None. M. Longmuir: None. L. Snadden: None. R. Davidson: None.

\section{EMP1.48D}

Measuring outcomes from genetic counselling: Establishing the minimum clinically important difference for the Genetic Counselling Outcome Scale

\section{Thomas ${ }^{1}$, M. McAllister ${ }^{2}$}

${ }^{1}$ Wessex Clinical Genetics Service, Southampton, United Kingdom, ${ }^{2}$ School of Medicine, Cardiff University, Cardiff, United Kingdom

Introduction: To establish the smallest change in genetic counselling outcome that is meaningful for patients, the aim of this study was to establish the Minimum Clinically Important Difference (MCID) for the Genetic Counselling Outcome Scale (GCOS-24). GCOS-24 is a patient-reported outcome measure for clinical genetics services. Secondary aims included understanding what patients deem important for reaching this score.

Materials and Methods: Participants were new patients recruited from the All Wales Medical Genetics Service. An anchor-based, global transition question methodology was used to identify the MCID, by asking participants how much meaningful change they experienced following their appointment, and comparing this with GCOS-24 change scores. The mean score of the group who felt 'a little better' was determined to be the MCID, using an independent samples t-test to compare with the group 'neutral'. This ensured that the established score was clinically meaningful to patients. Comments from a free text response box were analysed using qualitative thematic analysis.

Results: The response rate for completing GCOS-24, both before and after appointment, was approximately $10 \%$ $(n=53)$. Of these, $56.6 \%(n=30)$, also completed a free text response. The MCID was established to be a GCOS-24 score increase of 9 points after clinical attendance. This score was significantly different from the group 'neutral' $(\mathrm{p}=.003)$. Themes identified as important for reaching the MCID included 'future' and 'family'.

Conclusions: These findings contribute to interpretability of GCOS-24. Information provision alone was insufficient to ensure participants experienced clinical meaningful change, providing insights useful for genetic counselling service development.

C. Thomas: None. M. McAllister: None.

\section{EMP1.49A}

The Tell Me More Study: Returning medically actionable genomic variants in the setting of a neonatal genome sequencing study

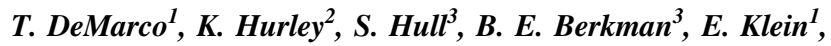
A. Fuller ${ }^{1}$, K. Huddleston ${ }^{1}$, B. Solomon ${ }^{1}$, F. M. Facio ${ }^{3}$

${ }^{1}$ Inova Translational Medicine Institute, Fairfax, VA, United States, ${ }^{2}$ Cleveland Clinic, Cleveland, OH, United States,

${ }^{3}$ National Human Genome Research Institute, Bethesda, MD, United States 
The Tell Me More Study (TMM) investigated parental decision-making about enrolling newborns in genome sequencing (GS) research. This qualitative study is part of the larger Inova Childhood Longitudinal Study at Inova Translational Medicine Institute. Through TMM, GS was performed concurrently for mother-father-child trios. Parents could learn GS results deemed medically-actionable for themselves and/or their child. We report a case of a 45-yearold Asian-American male found to carry a deleterious $B R C A 2$ truncating mutation previously reported in individuals of Filipino descent. GS analysis showed that his 3year-old daughter carries the BRCA2 mutation also; however, the parents were counseled to defer clinical confirmatory testing to an age when she can make the decision autonomously. Thus, only the father's result was confirmed in a CLIA-certified lab. An interview at 1-month post-disclosure indicated that while the father was initially surprised by the results and concerned about his daughter's risk, he felt that the information was empowering, allowing him and his at-risk relatives to participate in risk-reducing strategies. He reported sharing the information with relatives and a few colleagues, and intention to share it with his primary care provider. He envisioned sharing the results with his daughter when she is older and able to understand scientific principles. Despite learning this unexpected result, he did not regret his participation in the TMM study.

The case illustrates that people can adjust to and make use of unexpected GS results. However, it foreshadows new challenges in predictive testing for adult-onset conditions if GS in newborns is adopted more widely.

T. DeMarco: None. K. Hurley: None. S. Hull: None. B.E. Berkman: None. E. Klein: None. A. Fuller: None. K. Huddleston: None. B. Solomon: None. F.M. Facio: None.

\section{EMP1.50B}

Couples' communication about Huntington's disease: "The good, the bad, and the indifferent"

\section{J. Giblin, R. Dimond}

\section{Cardiff University, Cardiff, United Kingdom}

Facilitating family communication about inherited conditions is a crucial aspect of genetic counselling. Previous research has explored how families communicate about Huntington's Disease (HD). However, despite research demonstrating that partners are also greatly impacted by HD, communication between couples has largely been neglected. This qualitative study explored how individuals communicate about HD with family and friends, with particular focus on communication between couples. Semistructured interviews were conducted with nine participants who were either HD-positive, or the partner of someone who was HD-positive. In contrast with previous research, findings showed that whilst some participants reported they had not yet told their children and other relatives, all partners had been informed about the risk of HD. In most couples, the knowledge of HD was shared at a timely and suitable opportunity; either immediately after the risk was discovered, or early in the relationship. Thematic analysis indicated two styles of communication that continued after disclosure: (1) 'open communication' in which HD is freely and regularly discussed, and (2) 'pragmatic communication' in which HD is only discussed for practical reasons. Communication styles appeared to be related to discovering and disclosing the risk of HD, and to couples' coping strategies. This study provides novel insight into an underresearched subset of family communication, highlighting the role healthcare professionals can play in supporting disclosure to partners and facilitating ongoing discussion about HD between couples. The importance of this support cannot be understated when such communication is known to contribute to relationship stability and adjustment to a diagnosis.

J. Giblin: None. R. Dimond: None.

\section{EMP1.51C}

Genetic counselling in inherited retinal diseases: challenges associated with incomplete penetrance and variable expressivity

\section{Arasanz Armengol, G. Arno, A. Webster}

\section{Moorfields Eye Hospital, London, United Kingdom}

Purpose: To discuss the challenges associated with providing genetic counselling to families that have a mutation in an autosomal dominant gene with incomplete penetrance and variable expressivity, in the context of inherited retinal diseases.

Methods: We are describing six families that have been found to have mutations in CRX, PROM1 or PRPF31 through unbiased exome and genome sequencing. These genes are examples of genes that have been associated with cone dystrophy and rod cone dystrophy and show incomplete penetrance and variable expressivity. We exemplify the different challenges that arise as a result of mutations in non penetrant genes: assessing the recurrence risk based on a family history that looks autosomal recessive, discussing this risk with the patients and their families or discussing incomplete penetrance and its familial implications.

Discussion: Genetic counselling should be provided to every patient or family that is undergoing genetic testing. The aim should be to provide accurate, sufficient and 
comprehensible information along with psychological support to the patients. The complexity of genetics makes this process more challenging and incomplete penetrance is an example of this and of the importance of genetic counselling. It exemplifies how careful we must be in counselling prior and post gene sequencing.

M. Arasanz Armengol: None. G. Arno: None. A. Webster: None.

\section{EMP1.52D}

Parents' experiences of array comparative genomic hybridisation tests, when requested by a paediatrician

\section{B. A. Burns ${ }^{1}$, H. Shipman ${ }^{2}$, C. Kirwan ${ }^{3}$, S. Davies ${ }^{3}$, A. Clarke $^{3}$, R. Kirby ${ }^{4}$}

${ }^{1}$ Wessex Clinical Genetics, Southampton, United Kingdom, ${ }^{2}$ East Anglian Medical Genetics Service, Cambridge, United Kingdom, ${ }^{3}$ All Wales Medical Genetic Service, Cardiff, United Kingdom, ${ }^{4}$ All Wales Medical Genetic Service, Betsi Cadwaladr University Health Board, Rhyl, United Kingdom

Introduction: Array comparative genomic hybridisation (array CGH) is increasingly being used in the clinical setting. The range of potential results, particularly uncertain results, pose challenges for the requesting clinician. Due to mainstreaming, professionals outside the genetics service are increasingly requesting genetic tests. Lack of genetic knowledge and understanding in such professionals may heighten these challenges.

Materials and Methods: Three face-to-face interviews were carried out and audio-recorded. Interview transcripts were created and analysed using thematic analysis. Themes were discussed in relation to current literature.

Results: Overall participants' experiences were positive and the level of information given was reported as adequate. Little information was provided prior to the test being carried out and upon receiving the test result. Detailed information was later provided by the genetics service. Participants reported that they felt this was appropriate and the level of information provided at each time point was satisfactory.

Conclusion: Limited pre-test counselling is contradictory to the literature which emphasises the importance of detailed pre-test discussions. The literature also highlights concerns of mainstreaming where non-genetic healthcare professionals request genetic tests and in particular the potential negative implications this can have for patients. However, all participants were glad that the Paediatrician was the one to request the test and reported that they do not feel they would have further benefited from detailed pre-test counselling. This study highlighted the lack of research and information in the literature regarding this topic and the importance of such studies as mainstreaming is being increasingly implemented in medicine.

B.A. Burns: None. H. Shipman: None. C. Kirwan: None. S. Davies: None. A. Clarke: None. R. Kirby: None.

\section{EMP1.53A}

Parental considerations about the communication of myotonic dystrophy to their children

Z. D'Souza ${ }^{1,2}$, D. Scotcher ${ }^{1,2}$

${ }^{1}$ University of Manchester, Manchester, United Kingdom,

${ }^{2}$ Manchester Centre for Genomic Medicine, St Mary's Hospital, Manchester University NHS Foundation Trust, Manchester, United Kingdom

Communication about genetic conditions differs from other health problems, in that parents face the challenges of discussing the condition, its treatment and prognosis, and also the risk to their offspring. This communication process can be daunting for parents, who may feel ill-equipped and unsupported in this task.

Myotonic Dystrophy (DM) is a dominant genetic condition affecting multiple organ systems. Symptoms of DM are variable, and may cause problems from birth, in childhood or adulthood. Although DM is generally well researched, there is limited data about how parents with DM tell their children about their $50 \%$ risk of inheriting the condition.

This research study explores how parents with DM, and their partners, communicate, or contemplate communicating the risk of DM to their children. The study focusses on the facilitators and the barriers to this discussion, and the support from genetic services that parents could benefit from during this disclosure.

Eight participants were interviewed, via semi-structured interviews; and transcripts were analysed using Interpretive Phenomenological Analysis. Superordinate themes identified parents' emotions on having this discussion about the risk of DM, facilitators and barriers to disclosure, and support from genetic services that parents may value during this disclosure process.

There is currently no framework to help with the psychosocial impact of disclosure of risk of DM. By highlighting the personal experiences of parents in DMfamilies, this study has the potential to help genetics services frame a guidance strategy that addresses concerns, and helps support other parents who may face similar challenges in the future.

Z. D'Souza: None. D. Scotcher: None.

\section{EMP1.54B}

Three patients with pyridoxine-dependent epilepsy - 
psychological, ethical and professional issues in diagnostic approach as a proof of importance of pre and post-test genetic counseling

M. Mijovic ${ }^{1}$, A. Miletic ${ }^{1}$, B. Dimitrijevic ${ }^{1}$, J. Ruml Stojanovic ${ }^{1}$, M. Zivanovic ${ }^{1}$, G. Cuturilo C,2 $^{1,2}$

${ }^{1}$ University Children's Hospital, Department of Medical Genetics, Belgrade, Serbia, ${ }^{2}$ Faculty of Medicine, University of Belgrade, Belgrade, Serbia

Introduction: The era of genomic testing has led to new standards regarding genetic counseling.

Materials, Methods and Results: In the period of last four years we had seven patients with pyridoxine or pyridoxal phosphate-responsive epilepsy. Three of those are represented in the table.

\begin{tabular}{|c|c|c|c|c|c|}
\hline Patient & Specificity & Methods & Gene & Variant type & $\begin{array}{l}\text { Dilemmas } \\
\& \text { Issues }\end{array}$ \\
\hline 1. & $\begin{array}{l}\text { clear clinical } \\
\text { diagnosis }\end{array}$ & $\begin{array}{l}\text { Sanger } \\
\text { sequencing }\end{array}$ & PNPO & $\begin{array}{l}\text { c. } 520 \mathrm{C}>\mathrm{T} \text { (mat) } \\
\text { c. } 674 \mathrm{G}<\mathrm{A}(?)\end{array}$ & $\begin{array}{l}\text { misattributed } \\
\text { paternity }\end{array}$ \\
\hline 2. & $\begin{array}{l}\text { atypical } \\
\text { presentation } \\
\text { infant died }\end{array}$ & $\begin{array}{l}\text { Exome } \\
\text { sequencing }\end{array}$ & $A L D H 7 A 1$ & $\begin{array}{l}\text { c. } 328 \mathrm{C}>\mathrm{T} \text { (mat) } \\
\text { c. } 1566- \\
1 \mathrm{G}>\mathrm{T} \text { (pat) }\end{array}$ & late diagnosis \\
\hline 3. & $\begin{array}{l}\text { suspect } \\
\text { metabolic } \\
\text { disorders } \\
\text { mother } \\
\text { pregnant }\end{array}$ & $\begin{array}{l}\text { Exome \& } \\
\text { Mitochondrial } \\
\text { Genome } \\
\text { sequencing }\end{array}$ & $\begin{array}{l}P N P O \\
M T A T P 6 \\
\text { and } \\
\text { MTATP8 }\end{array}$ & $\begin{array}{l}\text { homozygot } \\
\text { c. } 674 \mathrm{G}>\mathrm{A} \\
\text { homoplasmia }\end{array}$ & $\begin{array}{l}\text { consanguinity } \\
\text { comorbidity } \\
\text { prenatal } \\
\text { testing }\end{array}$ \\
\hline
\end{tabular}

In the first case we had a dilemma about the best way of genetic counseling when discovering a suspicion of misattributed paternity. The second patient had an atypical clinical presentation, and unfortunately neurologist did not suspect this disease with available therapy. The patient from a third family is from an ethnically closed community, and the homozygous variant of the disease arouses suspicion of consanguinity. For this family, we also considered possible comorbidity, as well as prenatal diagnosis in advanced pregnancy.

Conclusion: In order to successfully overcome different ethical, psychological and professional issues and provide the best service to patients it is essential to have optimal pre and post-test genetic counseling.

M. Mijovic: None. A. Miletic: None. B. Dimitrijevic: None. J. Ruml Stojanovic: None. M. Zivanovic: None. G. Cuturilo: None.

\section{EMP1.55C}

Recurrence numbers in genetic counselling: exploring patient uptake, perception, and impact on clinical outcomes

K. Borle, E. Morris, A. Inglis, J. Austin

University of British Columbia, Vancouver, BC, Canada
Purpose: The provision of recurrence numbers is often considered a fundamental component of genetic counselling. We sought to fill knowledge gaps regarding how often patients request recurrence numbers, and how receiving numbers impacts patient outcomes.

Methods: We conducted a retrospective chart review at a clinic where patients routinely complete the Genetic Counselling Outcomes Scale [GCOS-24, measuring empowerment] pre (T1)/post-appointment (T2). We used ANCOVA to evaluate the effect of a) receiving recurrence numbers and b) patient perception of recurrence numbers, on patient outcomes at $\mathrm{T} 2$.

Results: Recurrence numbers were a primary indication for 134 of 300 patients $(45 \%)$. Some of these patients decided to not receive recurrence numbers after counseling about etiology and risk-reducing strategies $(\mathrm{N}=36)$, and recurrence numbers were ultimately provided to $116(39 \%)$ patients, most of whom $(n=64,55 \%)$ perceived the number to be lower than expected. There was no difference between GCOS-24 scores of those who received recurrence numbers and those who did not. However, a subset of patients - those who decided to not receive recurrence numbers despite it being an initial primary indication for the appointment - had greater increases in GCOS-24 scores. Perception of recurrence numbers had no impact on GCOS24 scores.

Conclusion: Our data challenge the notions that patients should routinely be provided recurrence numbers in genetic counselling, and that optimal patient outcomes of genetic counselling are contingent on receipt of recurrence numbers.

K. Borle: None. E. Morris: A. Employment (full or parttime); Significant; Provincial Health Services Authority. A. Inglis: A. Employment (full or part-time); Significant; Provincial Health Services Authority. J. Austin: A. Employment (full or part-time); Significant; BC Mental Health and Addictions institute. B. Research Grant (principal investigator, collaborator or consultant and pending grants as well as grants already received); Significant; Canadian Institutes of Health Research, Genome Canada.

\section{EMP1.56D}

Clinical uncertainty and familial interactions in the inherited cardiac conditions clinic in Hong Kong: reconsidering individualised notions of autonomy

\section{H. Shipman ${ }^{1}$, O. Zayts ${ }^{1}$, B. Chung ${ }^{2}$}

${ }^{1}$ School of English, Hong Kong, Hong Kong, ${ }^{2}$ Department of Paediatrics and Adolescent Medicine, Li Ka Shing Faculty of Medicine, The University of Hong Kong, Hong Kong, Hong Kong 
Introduction: The last twenty years have seen a number of empirical sociological and linguistic studies examining the process of genetic counselling (for both diagnostic and predictive testing), mostly in western contexts (Pilnick 2002; Sarangi 2016), but also more recently in non-western (Pilnick and Zayts 2014). Now, the changing technological environment (gene panel tests and genome wide testing) has cast increasing levels of uncertainty regarding diagnostic testing results and again established a need to genetically test other family members to substantiate a meaningful diagnostic genetic result. This development increasingly challenges Western ideologies of an individualistic model of informed consent to genetic testing, adding strength to the long-recognised notion that genetics is a 'family affair' (Hallowell 1999, 2009).

Methods and Results: We present discourse data from an inherited cardiac conditions clinic in Hong Kong, where several family members are co-present. Drawing on Goffman's participation framework (1981) we examine the process of giving and receiving clinically uncertain results from a gene panel test and negotiating ongoing testing within the family. We explore how this test blurs the boundaries between diagnostic and ongoing genetic testing within one family and leads the researcher to re-consider the question 'who is the patient?', the individual undergoing the testing or the family.

Conclusions: We consider the implications of our results for Hong Kong, where genetic counselling is being established and where Western and Eastern cultures meet (Zayts et al. 2013) and beyond, in challenging individualised notions of autonomous decision-making and informed consent.

H. Shipman: None. O. Zayts: None. B. Chung: None.

\section{EMP1.57A}

\section{Disclosing a Diagnosis of Von Hippel-Lindau Disease to Affected Children: Parental Experiences and Views}

\section{S. A. Hamilton ${ }^{1,2}$, D. Scotcher ${ }^{1,3}$}

${ }^{1}$ Division of Evolution and Genomic Sciences, School of Biological Sciences, University of Manchester, Manchester, United Kingdom, ${ }^{2}$ North of Scotland Genetic Service, Aberdeen Royal Infirmary, NHS Grampian, Aberdeen, United Kingdom, ${ }^{3}$ Manchester Centre for Genomic Medicine, St Mary's Hospital, Central Manchester University Hospitals NHS Foundation Trust, Manchester Academic Health Sciences Centre, Manchester, United Kingdom

Von Hippel-Lindau Disease (VHL) is a dominantly inherited tumour predisposition syndrome in which manifestations can potentially occur in childhood. As screening can commence from age five, predictive genetic testing is offered at a young age, leaving parents responsible for informing their children of the diagnosis. This study attempts to address how parents disclose a diagnosis of VHL to their affected children. Parents of affected children were identified through the North West Genetic Service VHL Register and were invited to participate in semistructured telephone interviews. Four parents agreed to participate and interview data was analysed using Interpretive Phenomenological Analysis. Three themes were identified in the data; the disclosure process, parental experiences and support and challenges of VHL. The research showed that the aim of disclosure was to acknowledge the significance of VHL while minimising the negative impact it may have. A novel finding was that disclosure conversations tended to happen around the time of screening appointments and considerations made by parents were similar to what has been reported for other genetic conditions. Both the affected and unaffected parent played a significant role in disclosure and support of a genetic counsellor was seen as beneficial. There were many challenges specific to VHL disclosure, most prominently a lack of child-friendly information. The research showed that current advice given to parents is appropriate and support from genetic counsellors should continue. However childfriendly information should be developed so that genetic counsellors can better support families affected by VHL.

S.A. Hamilton: None. D. Scotcher: None.

\section{EMP1.58B}

Family management of life with Down syndrome in the era of noninvasive prenatal testing

\section{L. Van Riper, G. J. Knafl, K. A. Knafl}

University of North Carolina Chapel Hill, Chapel Hill, NC, United States

Introduction: There is growing evidence that many families successfully manage the ongoing challenges associated with raising an individual with Down syndrome (DS). Ironically, widespread use of noninvasive prenatal testing (NIPT), appears to be exacerbating some of the challenges faced by families of individuals with DS (e.g., stigma, discrimination, access to services). The purpose of this study was to explore cross-cultural differences in family management of DS.

Method: 2740 parents of individuals with DS (2387 mothers and 353 fathers) completed a 53-item Family Management Measure as part of a large cross-cultural study concerning adaptation and resilience in families of individuals with DS. Means for each of the six subscales (Condition Management Ability, Child's Daily Life, Family Life Difficulty, Condition Management Effort, View of 
Condition Impact, and Parental Mutuality) were ranked by country.

Results: Parents from Portugal, Spain and the US had mean scores that ranked 6 or below for all the subscales indicating greater ease in family management, while parents from Ireland, Italy, Korea, and Thailand had mean scores that ranked 6 or above for all the subscales indicating more problematic family management. For the remaining three countries (Brazil, Netherlands, and United Kingdom), rankings on the subscales were mixed.

Conclusion: Findings from this study suggest there are cross-cultural differences in family management of DS. More research is needed to fully understand if these differences are related to social determinants of health such as culture, societal attitudes towards DS and national approaches to integrating NIPT into clinical practice.

M.L. Van Riper: None. G.J. Knafl: None. K.A. Knafl: None.

\section{EMP1.60D}

Black South African women's perspectives on the impact of having a child with a genetic disorder on their spousal relationship

\section{T. S. Haw ${ }^{1}$, S. Henriques ${ }^{2}$}

${ }^{1}$ National Health Laboratory Service, Johannesburg, South Africa, ${ }^{2}$ Manchester Centre for Genomic Medicine, Manchester, United Kingdom

South Africa has high levels of poverty with an unemployment rate of $25 \%$. Census studies have shown that of children aged 0-4 years old in South Africa, only $36 \%$ live with both parents. Very little is known about how having a baby with a genetic condition impacts on the spousal relationship in an already fragmented society. This study examined the impact that having a child with a genetic condition has on the spousal relationship from the perspective of black South African women in state healthcare. Eight mothers who have a child with a genetic disorder were invited to take part in this study while attending a Genetic Counselling Clinic in a state funded hospital in Johannesburg, South Africa. Data was collected through semi-structured interviews lasting about 30 minutes. Each participant's personal experiences and how they made sense of them were analysed using thematic analysis. Three main themes were identified during the study including blame, the importance of spousal and family support and the spouse's reaction to the child. The study highlights the need to provide support to the spousal relationship when a genetic diagnosis is made, perhaps by increasing efforts to include the male spouse in the genetic counselling session. Understanding the impact that having a child with a genetic disorder has on the spousal relationship can assist genetic counsellors to provide more focused and empathic support for women and couples in this situation in South Africa.

T.S. Haw: None. S. Henriques: None.

\section{EMP1.61A}

An eclectic approach to decision-making in genetic counselling, incorporating person-centred, motivational interviewing and skilled helper models

\section{H. M. Brandon, K. Kohut, E. Winchester}

St George's University Hospitals NHS Foundation Trust, London, United Kingdom

Background: There is no single model of genetic counselling that will suit all clients due to unique differences, diverse preferences and needs.

Method: To meet client needs, a technical eclecticism approach to genetic counselling has been developed, primarily consisting of Roger's person-centred counselling, motivational interviewing and Egan's skilled helper model.

Discussion: Roger's person-centred model was initially adopted in genetic counselling to separate the profession from eugenics. Whilst empathy, unconditional positive regard and congruence mean clients are supported to explore issues and make their own decisions, they can avoid difficult issues. Combining with other models can improve decision-making.

Motivational interviewing aims to explore ambivalence, support self-efficacy and when the client is ready, initiate change plans, whilst maintaining empathy. It can be particularly helpful in assisting clients to resolve ambivalence using active counselling techniques, such as highlighting discrepancies and affirmations for empowerment.

Egan's Skilled helper model outlines a systematic decision-making plan which integrates with skills, tools and techniques from other models, providing an ideal framework for an eclectic approach.

Results and conclusion: An eclectic approach means that clients' goals, blind spots and options are explored with a variety of counselling skills, before the client chooses their 'best fit' option, minimizing possible post-decision regret. The practical application of this approach will be demonstrated with a decision-making case exemplar.

H.M. Brandon: None. K. Kohut: None. E. Winchester: None.

\section{EMP1.62B}

Family burden of children suffering from Epidermolysis Bullosa 
S. De Stefano ${ }^{1}$, F. Lalatta ${ }^{2}$, G. Tadini ${ }^{3}$, F. S. Grassi ${ }^{4}$, D. Rossetti ${ }^{4}$, S. Salera ${ }^{4}$, M. Brena $^{5}$, A. Lopopolo ${ }^{6}$, O. Picciolini ${ }^{6}$, A. Spinelli, G. Montani ${ }^{2}$, G. Scuvera ${ }^{4}$, S. Guez ${ }^{8}$

${ }^{1}$ Clinical Genetics Unit Fondazione IRCCS Cà Granda Ospedale Maggiore Policlinico, MILANO, Italy, ${ }^{2}$ Clinical Genetic Unit Fondazione IRCCS Cà Granda Ospedale Maggiore Policlinico, MILANO, Italy, ${ }^{3}$ Pediatric dermatology unit Fondazione IRCCS Cà Granda Ospedale Maggiore Policlinico, MILANO, Italy, ${ }^{4}$ Pediatric Highly Intensive Care Unit Fondazione IRCCS Cà Granda Ospedale Maggiore Policlinico, MILANO, Italy, ${ }^{5}$ Pediatric Dermatology Unit Fondazione IRCCS Cà Granda Ospedale Maggiore Policlinico, MILANO, Italy, ${ }^{6}$ Pediatric Physical Medicine and Rehabilitation Unit, Fondazione IRCCS Cà Granda Ospedale Maggiore Policlinico, MILANO, Italy, ${ }^{7}$ Social service unit Fondazione IRCCS Cà Granda Ospedale Maggiore Policlinico, MILANO, Italy, ${ }^{8}$ Pediatric Highly Intensive Care Unit, Fondazione IRCCS Cà Granda Ospedale Maggiore Policlinico, MILANO, Italy

Introduction: Few studies have considered the psychosocial impact of Epidermolysis Bullosa on affected children and their families.

Cases and methods: $50 \mathrm{~EB}$ families were recruited to answer a 20 item questionnaire to assess the burden on their families and their adaptation to the disease. The diagnosis of EB was based on clinical observation, on histopathological analysis and/or molecular testing. The cohort consisted of 13 cases of EBS, 3 of JEB, 32 of DEB (11 DDEB and 21 RDEB) and 2 cases of KS. Disease severity was evaluated using the Birmingham Epidermolysis Bullosa Severity (BEBS) score. The questionnaire demonstrated high internal consistency (Cronbach's alpha is 0,90) and the EB Burden of Disease (EB-BoD) score was well correlated with the mental HRQoL outcome of the SF-12, confirming its concurrent validity. The interclass correlation coefficient (ICC) was 0.97 while EB-BoD scores were significantly discriminating between EB subtypes. The survey included evaluations of family life, the child's well-being, disease and treatment as well as economic and social impact. The format included a six-point Likert scale. Further data included family composition, demographic data and sociocultural aspects of the family members.

Result: all parents answered the questionnaire. Preliminary results revealed that there is not strict correlation between BEBS scores and levels of burden. There are psychological, socioeconomic and medical aspects that influence the entire family, regardless of the severity of the condition. So, it is important to improve our knowledge and our ability to measure these aspects to set priorities for children and families.
S. De Stefano: None. F. Lalatta: None. G. Tadini: None. F.S. Grassi: None. D. Rossetti: None. S. Salera: None. M. Brena: None. A. Lopopolo: None. O. Picciolini: None. A. Spinelli: None. G. Montani: None. G. Scuvera: None. S. Guez: None.

\section{EMP1.65A}

BIUX^2X2 $^{\wedge}$

\author{
A. Ros, B. García-Jiménez, E. Castellanos, F. Roca-Ribas, \\ E. Amilibia, A. Castillo, J. L. Becerra, I. Bielsa, A. Plana, \\ C. Lázaro, E. Serra, I. Blanco
}

Spanish National Reference Centre (CSUR) for Phakomatoses, Badalona, Spain

A pilot study was performed to validate and adapt the original NF2 Impact on Quality of Life (NFTI-QOL) questionnaire to assess Neurofibromatosis 2 (NF2) Spanish patients. Our previous pilot results showed low internal consistence. The aim of the present study was to improve the cross-cultural adaptation and validation of the Spanish version of NFTI-QOL Scale.

Patients and methods: A new translation, retrotranslation of the NFTI-QOL to Spanish was performed to optimize the structure of the answers. Validation of the Spanish scale was performed by means of the factorial analysis of principal components and the use of a second generic QoL questionnaire (EuroQOL) in a sample of 23 patients with NF2, under care of Spanish National Reference Centre for Phakomatoses.

Results: The participants were 7 males and 16 females with an age rate of 17 to 77 years. Results did not show statistically significant differences by gender $(p>0.01)$ and did not correlate with age $(r=0.287)$. The new translated version showed good internal consistence (Cronbach's $\alpha=$ 0.86). The analysis of principal factor showed a bidimensional structure of the scale $(\mathrm{KMO}=0,716$, Bartlett test $\mathrm{p}<$ 0.01). The total NFTI-QOL score correlated significantly with total score of the five items of EuroQOL $(r=-0,857$, $\mathrm{p}<0.001)$. Mean NFTI-QOL score obtained was 9,57.

Conclusions: The modified version of the translated NFTI-QOL scale has better internal validity and reliability. It is a validated scale to evaluate the QoL in Spanish NF2 patients. Ref: Hornigold et al. J Neurol Surg B 2012;73:104-111.

A. Ros: None. B. García-Jiménez: None. E. Castellanos: None. F. Roca-Ribas: None. E. Amilibia: None. A. Castillo: None. J.L. Becerra: None. I. Bielsa: None. A. Plana: None. C. Lázaro: None. E. Serra: None. I. Blanco: None. 


\section{EMP1.66B}

'We're more defined by what we are not than what we are': Exploring adoptees' attitudes and experiences of family health history and genetic information

\section{T. R. O'Brien ${ }^{1,2}$, J. Hodgson ${ }^{1,2}$, M. Sahhar ${ }^{3}$, K. $_{\text {Pereira }}^{4,3}$ \\ ${ }^{1}$ The University of Melbourne, Melbourne, Australia, \\ ${ }^{2}$ Murdoch Children's Research Institute, Melbourne, Australia, \\ ${ }^{3}$ Victorian Clinical Genetics Services, Melbourne, Australia, \\ ${ }^{4}$ The Genetic Support Network of Victoria, Melbourne, Australia}

Introduction: Due to the nature of adoption, people who are adopted often have limited access to family health history $(\mathrm{FHH})$ information. Although previous research has explored the perspectives of adoptive parents on limited FHH knowledge of their adopted child, little is known about how this impacts on adoptees themselves. Anecdotal reports suggest adoptees access direct-to-consumer (DTC) genetic testing to gain information about their inherited health risks. This study aimed to explore the attitudes and experiences of adoptees regarding $\mathrm{FHH}$ and genetic information, and interest in genetic testing.

Methods: This was a qualitative study, involving 10 semi-structured interviews with adoptees. Interviews were transcribed verbatim, coded and analysed using thematic analysis.

Results: The main findings of this research highlight the importance of the adoption experience in providing context to participant's attitudes to FHH and genetic information. The complex nature of facets of the adoption experience, including contact and relationships with birth family and the process of searching, illustrate potential psychosocial impacts for adoptees. This research confirms anecdotal reports that adoptees are accessing DTC testing to fill gaps in their knowledge. It provides novel insights into the potential interest and value of genetic testing for adoptees, such as not facing barriers that are common to other aspects of the adoption experience, and the perceived ability of genetic testing to uncover the 'truth'.

Conclusions: The findings of this research help to inform practice in providing genetic counselling to this group. They also demonstrate the importance of training for genetic counsellors in aspects of adoption.

T.R. O'Brien: None. J. Hodgson: None. M. Sahhar: None. K. Pereira: None.

\section{EMP1.67C}

Choosing not to know: accounts of non-engagement with pre-symptomatic testing for late onset-neurological disorders
Á. Mendes $^{1}$, M. Paneque ${ }^{1}$, A. Clarke $^{2}$, J. Sequeiros ${ }^{1,3}$

${ }^{1}$ i3S, IBMC - UnIGENe and CGPP, University of Porto, Porto, Portugal, ${ }^{2}$ School of Medicine, Institute of Medical Genetics, Cardiff University, Cardiff, United Kingdom, ${ }^{3}$ ICBAS Instituto de Ciências Biomédicas Abel Salazar, Universidade do Porto, Porto, Portugal

Few studies have addressed those who remain uninformed about their genetic status for their family's disease. We report the accounts from people at-risk or affected by Huntington disease, familial amyloid polyneuropathy TTR Val30Met and Machado-Joseph disease, and their family members, who have decided not to undertake presymptomatic testing (PST) for those diseases. We draw on individual semi-structured interviews, and on family interviews, involving a total of 21 participants recruited through patients' associations. Qualitative thematic analysis was undertaken. Findings show that some participants frame access to predictive genetic information as pointless because, although removing uncertainty, no effective treatment or cure is available for their family's disease. They also anticipate genetic knowledge as having the potential to become seriously burdensome psychologically. Other participants prioritize the focus of their lives on everyday pressing concerns, without the destabilizing knowledge of an impending disease. Some participants affirmed that by avoiding formal knowledge of their genetic status they are able to preserve hope towards the future. In doing so, they are allowed to live a "valid life". There were also accounts where ambivalence among family members in relation to non-engagement was noticeable. This study may be relevant for genetic counselling and for the provision of support to families, by bringing further insights about the reasoning of those who opt for non-engagement with medical genetic services, and specifically with PST, and into the decision-making process of at-risk family members.

Á. Mendes: None. M. Paneque: None. A. Clarke: None. J. Sequeiros: None.

\section{EMP1.68D}

Ectodermal Dysplasia: motivating and sustaining involvement in a trial involving newborn boys

\section{S. Doheny, A. Clarke}

Division of Cancer and Genetics, Cardiff, United Kingdom

In October 2013, Edimer initiated a phase 2 trial to determine the safety of administering the drug EDI200 to newborn males. While raising the possibility of treating Ectodermal Dysplasia (specifically, X-linked Hypohidrotic Ectodermal Dysplasia (XLHED)), involvement in this trial 
required a great deal of participating families. Not only did the eligible foetus have to be identified and screened to check their sex, but the newborns were required to move to a participating hospital in their first week after birth, where they were to spend nearly the first month of their lives. Making such a commitment to determine the safety of a drug, suggests that recruiting clinicians, patients and families at the very least share perceptions and values. Through an analysis of interviews conducted with the families of the infants, participating clinicians, and representatives of Edimer, we unpack the views and experiences of some of those involved in this trial. Driving the willingness to participate in the trial was a strong sense of the personal and social problems caused by Ectodermal Dysplasia, coupled with discourses on hope and scientific progress. What is noteworthy, however, is how this combination of discourses motivated and sustained involvement in a lengthy and burdensome trial.

S. Doheny: None. A. Clarke: None.

\section{EMP1.69A}

Ehlers-Danlos syndrome in the university hospital of Liege

\section{Kukor, S. Bertoli, V. Bours, S. Bulk, E. Docampo}

\section{CHU Liège, Liège, Belgium}

Introduction: Ehlers Danlos Syndrome (EDS) is a common reason for consultation in clinical genetics. In our genetic consultations, we are confronted with a large number of patients referred for joint hypermobility. Often, another practician has already established a diagnosis of hypermobility type EDS. The hypermobility type diagnosis is based on clinical findings only. Thus, the genetic consultation does not seem to be of additional value. The aim of this study was to characterize patients referred to clinical genetics consultations for EDS suspicion.

Methods: We performed a retrospective observational study of the patients from the University Hospital of Liège who had come to a genetic consultation for the EhlersDanlos syndrome between March 2016 and December 2017. We performed descriptive statistical analyses of demographic and clinical characteristics including Beighton score, Ehlers-Danlos syndrome associated clinical signs, family history, as well as the prescribed complementary tests.

Results: We included 143 patients. Except for rare cases, the vast majority of the evaluations only confirmed the already established diagnosis of EDS hypermobility type. A consultation in the genetics polyclinic was therefore not necessary for those patients.

Conclusion: The vast majority of the patients referred for an EDS suspicion presented a hypermobile type. Genetic consultation did not have any additional value. Since the diagnosis is based on clinical findings, it is essential to make other practitioners aware of this. Referral criteria should be defined in order to select cases needing a clinical genetic evaluation.

L. Kukor: None. S. Bertoli: None. V. Bours: None. S. Bulk: None. E. Docampo: None.

\section{EMP1.70B}

Motivations and expectations of those participating in the Enroll-HD observational study

${\text { E. } \text { Davies }^{1,2}, \text { R. MacLeod }}^{1,3}$

${ }^{1}$ Division of Evolution and Genomic Sciences, School of Biological Sciences, University of Manchester, Manchester, United Kingdom, ${ }^{2}$ Addenbrooke's Hospital, Cambridge University Hospitals, Cambridge, United Kingdom, ${ }^{3}$ Manchester Centre for Genomic Medicine, St Mary's Hospital, Central Manchester University Hospitals NHS Foundation Trust Manchester Academic Health Sciences Centre, Manchester, United Kingdom

Introduction: Predictive test guidelines for Huntington's disease recommend that individuals are offered opportunities to participate in research regardless of test outcome. In MCGM individuals are invited to participate in the observation study Enroll-HD. Limited research has been conducted to date on the views of research participants and the possible impact of participation on their lives. The aim of this study was to explore the experiences of individuals taking part in Enroll-HD study following predictive testing.

Materials and Methods: Ten individuals participating in Enroll-HD at MCGM agreed to a semi-structured telephone interview. All had undergone predictive testing for HD; five individuals tested mutation positive, and five individuals tested mutation negative. The interviews were audiotaped, transcribed verbatim and analysed using thematic analysis.

Results: Participants had both personal and altruistic motivations for taking part in Enroll-HD. Overall, they were very positive about their experiences, citing valuable aspects such as good relationships with the research team, increased understanding of $\mathrm{HD}$, an enhanced self-image and a shared experience with an affected relative. Issues highlighted were of a practical nature including distance travelled or car parking. Several participants would have welcomed more information about study progress. Participants generally had realistic expectations for the study, aware of the speed and limitations of research, but were also cautiously optimistic for the future.

Conclusions: Participants were overall positive about the experience of research participation. Understanding the motivations and possible barriers to taking part in research 
is essential to improve patient experience and to help retain study participants.

E. Davies: None. R. MacLeod: None.

\section{EMP1.71C}

The cost of juvenile onset Huntingdon's Disease (JOHD) in the UK

\section{T. A. Young ${ }^{1}$, O. W. J. Quarrell ${ }^{2}$ \\ ${ }^{1}$ University of Sheffield, Sheffield, United Kingdom, ${ }^{2}$ Sheffield Children's NHS Trust, Sheffield, United Kingdom}

Introduction: Huntington's disease patients with onset $\leq 20$ years account for $5 \%$ of cases. Estimates of the costs of HD have been published; to date no study has estimated the costs of JOHD. Methods: 33 people with JOHD or their families/carers were interviewed and completed a questionnaire on the use of resources in the past 12 months. Costs were estimated overall and for those with mild, moderate and severe JOHD.

Results: the mean age of the people with JOHD was 23 years: $52 \%$ were male. There were 10 mild, 7 moderate, 14 severe and two unclassified cases. The majority of costs incurred were due to accessing services. $50 \%$ of people accessed accident \& emergency mainly because of falls. The majority of costs borne by the person with JOHD or their family were for direct care $(£ 26,577)$. The mean overall cost of JOHD was $£ 112,581$ per person per year. The average cost to the NHS was $£ 69,368$ and $30 \%$ of the overall cost to the person with JOHD and their family (mean $=£ 34,758$ ). Overall costs were lowest for those with mild JOHD (mean $=£ 27,764$ ), and highest for those with severe JHD (mean - £181,523) with moderate JHD costing on average $£ 110,341$ per year.

Conclusions: A UK study on HD reported cost per year to be $£ 21,605$ and included similar resource use: the cost of JOHD is nearly five times more than this. [Research funded by the NHS National Institute for Health Research. Research for Patient Benefit stream. PB-PG-112-29056].

T.A. Young: None. O.W.J. Quarrell: None.

\section{EMP1.72D}

'They're not there for you': a qualitative study exploring the impact of service organisation on the psychosocial experiences of young people with juvenile onset Huntington's Disease, and their parents, in England

\author{
P. A. Curtis ${ }^{1}$, J. Thompson ${ }^{1}$, O. W. J. Quarrell ${ }^{2}$, H. Santini ${ }^{3}$ \\ ${ }^{1}$ Sheffield University, Sheffield, United Kingdom, ${ }^{2}$ Sheffield \\ Children's NHS Trust, Sheffield, United Kingdom, \\ ${ }^{3}$ Huntington's Disease Association, Liverpool, United Kingdom
}

Introduction: Previous studies examining family carers' experiences of support services have highlighted the role of interpersonal factors such as trust, honesty and the importance of listening to family members. However, little is known about how service organisation issues impact upon family members' psychosocial experiences and, more broadly, their perceptions of effective support. Aim: To identify key components of effective support from the perspectives of young people with Huntington's Disease and their family carers.

Methods: The paper presents findings from qualitative data generated within a multiphase, mixed-methods study. Multiple strategies (including referrals from clinical genetics centres and from the Huntington Disease Association) were utilised to identify families. 24 family interviews were conducted, of which 9 included young people with JOHD. Data was subjected to framework analysis.

Results: For many families, formal support services are experienced as sub-optimal, with consequent negative impact on families' psychosocial experiences. Seven core components of effective support were identified. These were: proactivity; accessibility and ease of communication; positive interpersonal relationships; continuity of relationships; partnership working; specialist knowledge of JHD and; access to a key worker.

Conclusion: There is considerable potential to improve the support provided to young people with JHD and their family carers. Key to such improvement is the allocation of a key worker with whom family members can initiate and maintain contact over time as the disease progresses, the young person's health deteriorates and support needs change. [Research funded by the NHS National Institute for Health Research. Research for Patient Benefit stream. PB-PG-112-29056].

P.A. Curtis: None. J. Thompson: None. O.W.J. Quarrell: None. H. Santini: None.

\section{EMP1.73A}

Juvenile onset Huntington's disease: The health status and perspective of family carers

\section{A. K. Ho ${ }^{1}$, O. W. J. Quarrell}

${ }^{1}$ University of Reading, Reading, United Kingdom, ${ }^{2}$ Sheffield Children's NHS Trust, Sheffield, United Kingdom

Introduction: Juvenile onset Huntington's disease (JOHD) is defined as onset $\leq 20$ years. Although most patients are cared for within the family, the experiences of family-carers of JOHD is lacking. This study explores the perspective of JOHD family-carers together with their health status.

Methods: Twenty six JOHD family-carers (with an average age 45 years) completed the EQ-5D questionnaire. 
They were also asked about their experience as carers and their responses were analysed qualitatively.

Results: Family-carers reported positive elements, which were growth in the carer's character, strength of the JOHD patient's character, enriched family experiences, and supportive external relationships. Negative points reported were the personal and emotional toll on carers, toll on the family, and issues with external parties regarding JOHD. Carers' responses on what would be helpful centred on financial support, respite, and an increase in both the quantity and quality of services appropriate for JOHD. Family-carers' health status was lower than normal for their age group (EQ-5D visual analogue scale rating $=73$, EQ5$\mathrm{D}$ index score $=0.74)$. Pain/Discomfort was most affected in family-carers, followed by Mobility, with poorer scores for those caring for more severely affected JOHD patients.

Conclusions: Family-carers face a high physical and emotional toll, especially when JOHD patients become severely affected. Greater understanding and acknowledgement of this is needed to address the health and quality of life of carers. [Research funded by the NHS National Institute for Health Research. Research for Patient Benefit stream. PB-PG-112-29056].

A.K. Ho: None. O.W.J. Quarrell: None.

\section{EMP1.74B}

\section{Exploring the role of religion and spirituality on Muslim} patients with Long QT Syndrome

$$
\text { K. Bakur }{ }^{1,2}, \text { F. Ulph }{ }^{1}, \text { H. Brooks }{ }^{1,3}, \text { T. } \text { Clancy }^{1,4}
$$

${ }^{1}$ University of Manchester, Manchester, United Kingdom, ${ }^{2}$ King Abdulaziz University, Jeddah, Saudi Arabia, ${ }^{3}$ University of Liverpool, Liverpool, United Kingdom, ${ }^{4}$ Manchester Centre for Genomic Medicine, Manchester University Hospitals NHS Foundation Trust, Manchester, United Kingdom

Introduction: Individuals' religious beliefs and spirituality $(\mathrm{R} / \mathrm{S})$ can influence their experience with a personal and/or family history of a genetic disorder. The aim of this qualitative study is to explore the role of religion and spirituality on Muslim patients with Long QT Syndrome.

Materials and Methods: To date, 7 participants have been recruited through a cardio-genetic clinic in Saudi Arabia. Interviews have been analysed using IPA.

Preliminary Results: All participants felt that their faith helped them overcome difficult situations in general and the genetic condition in particular. On the other hand, they also believed that difficult situations strengthened their relation with Allah (God). Participants viewed their diagnoses through the lens of religious belief, but did not consider it to be a punishment from God. Although participants attribute ultimate knowledge to Allah, they confidently trust the medical knowledge and balanced between their religious belief and adjustment to the diagnosis being seen as a duty. Belief in fate was a primary coping strategy to overcome difficult situations.

Conclusion: Muslim patients use R/S as a coping strategy in the context of LQTS. However, genetic counsellors should explore individualised beliefs around R/S since people vary in their interpretations of religious principles. Patients who believed fate is predetermined focus more on controlling how they respond to life events.

K. Bakur: None. F. Ulph: None. H. Brooks: None. T. Clancy: None.

\section{EMP1.76D}

"I just wanted answers": Exploring personal genomic testing for nutrition and wellness

E. Tutty ${ }^{1,2}$, C. Hickerton ${ }^{2,1}$, M. Adamski ${ }^{3}$, J. Savard ${ }^{4,5}$,

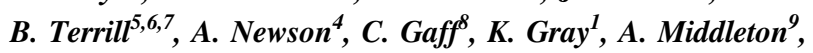
B. Wilson ${ }^{10}$, S. Metcalfe ${ }^{1,2}$

${ }^{1}$ The University of Melbourne, Melbourne, Australia, ${ }^{2}$ Murdoch Childrens Research Institute, Melbourne, Australia, ${ }^{3}$ Monash University, Melbourne, Australia, ${ }^{4}$ The University of Sydney, Sydney, Australia, ${ }^{5}$ Garvan Institute of Medical Research, Sydney, Australia, ${ }^{6}$ Genome.One, Sydney, Australia, ${ }^{7}$ The University of New South Wales, Sydney, Australia,

${ }^{8}$ Melbourne Genomics Health Alliance, Melbourne, Australia, ${ }^{9}$ Society and Ethics Research, Connecting Science, Welcome Genome Campus, Cambridge, United Kingdom, ${ }^{10}$ The University of Ottawa, Ottawa, ON, Canada

Personal genomic testing (PGT) for nutrition and wellness offers new ways to personalise dietary advice. While promising, commercial adoption of nutritional genomics may be hasty: many tests lack clinical validity and utility (e.g. MTHFR). Nevertheless, companies and complementary/ alternative health practitioners (e.g. naturopaths/nutritionists) encouraging PGT for nutrition and wellness online have increased, particularly in Australia; little is known about peoples' experiences of this. We aimed to explore how PGT for nutrition and wellness is framed online to the public and how Australians experience testing. This study involved: content analysis of websites $(n=39)$ that promote PGT services for nutrition and wellness to Australians, using a newly created framework; and thematic analysis of semi-structured interviews $(\mathrm{n}=12)$ with participants (from Genioz study survey) who had testing. Websites were framed to empower the consumer, claiming testing as an essential tool for optimising health, with little emphasis on scientific and ethical aspects. Most interviewees experienced chronic ill-health and found PGT was not as informative as promised nor easy to translate into real life. 
However, participants remained positive about PGT and valued the involvement of a complementary/alternative health practitioner following negative experiences with conventional medical professionals regarding testing and their condition. Despite concerns regarding clinical validity and utility, findings revealed that PGT for nutrition and wellness provides the chronically ill with self-knowledge and a sense of empowerment. The healthy public, however, may be more prone to disappointment and psychological distress. Clearly, greater accuracy of information, and adequate pre- and post-test counselling, is necessary to facilitate informed consent.

E. Tutty: None. C. Hickerton: None. M. Adamski: None. J. Savard: None. B. Terrill: Other; Modest; Genome.One. A. Newson: None. C. Gaff: None. K. Gray: None. A. Middleton: None. B. Wilson: None. S. Metcalfe: None.

\section{EMP1.78B}

Psychosocial effects on a genetic disease-Investigating the psychological resilience of adults living with sickle cell disease

C. I. Conn ${ }^{1}$, L. Jackson ${ }^{2}$, N. S. Trivedi ${ }^{1}$, A. Buscetta ${ }^{1}$, K. Abdallah ${ }^{1}$, K. D. Blizinsky ${ }^{3}$, C. P. Minniti ${ }^{4}$, V. L. Bonham ${ }^{1}$

${ }^{1}$ National Human Genome Research Institute, Bethesda, MD, United States, ${ }^{2}$ Pediatrics and Child Health, College of Medicine, Howard University, Washington D.C, DC, United States, ${ }^{3}$ Rush Alzheimer's Disease Center, Rush University, Chicago, IL, United States, ${ }^{4}$ Department of Medicine, Division of Hematology, Albert Einstein College of Medicine,

Montefiore Medical Center, Bronx, NY, United States

Introduction: Sickle cell disease (SCD) affects about 100,000 people in the U.S. Its frequency is steadily rising in Europe with immigration. Psychological resilience is considered a characteristic of healthy patients living with chronic diseases, but has rarely been explored in adults living with SCD. The Brief Resilience Scale (BRS) measures an "individual's ability to bounce back from stress." Using BRS, we sought to explore what factors influence individuals' resilience while living with SCD and how resilience relates to these individuals' physical and psychosocial wellbeing.

Methods: A cross-sectional study using psychosocial, physiological, and environmental data within U.S. Participants $(\mathrm{N}=102)$ with the highest and lowest quartiles of BRS scores were identified. Student's t-test or chi-square analysis was conducted between data on participants in the distal quartiles. Spearman correlations were calculated between BRS scores and all data variables for the overall cohort and the distal quartiles. A cutoff of \pm 0.3 with $p$-value $\leq 0.05$, was used to determine significance for correlation coefficients.

Results: BRS was associated with multiple psychosocial measures for both the overall cohort and quartiles e.g. Cohen Perceived Stress Scale, $-0.55, \mathrm{p}<0.01$. BRS was not associated to any physiological measures, such as white blood cell count or hematocrit levels, in the overall cohort.

Conclusions: Our findings suggest that BRS is strongly linked to psychosocial measures and is not correlated with physiological disease severity. Interventions targeting the resilience of individuals with a chronic genetic disease needs to involve psychosocial factors. This study is funded by NHGRI, NIH, United States, 1ZIAHG200394-04

C.I. Conn: None. L. Jackson: None. N.S. Trivedi: None. A. Buscetta: None. K. Abdallah: None. K.D. Blizinsky: None. C.P. Minniti: None. V.L. Bonham: None.

\section{EMP1.79C}

Interrelation of smoking with neuroendocrine and psychological phenotypes in young men from the Sakha Republic of Russia

S. S. Nakhodkin', N. A. Barashkov ${ }^{1,2}$, V. G. Pshennikova ${ }^{1,2}$, A. V. Kazantseva ${ }^{3}$, E. K. Khusnutdinova ${ }^{3,4}$, N. N. Sazonov ${ }^{1}$, S. A. Fedorova $^{1,2}$

${ }^{1}$ M.K. Ammosov North-Eastern Federal University, Yakutsk, Russian Federation, ${ }^{2}$ Yakut Science Centre of Complex Medical Problems, Yakutsk, Russian Federation, ${ }^{3}$ Institute of Biochemistry and Genetics, Ufa, Russian Federation, ${ }^{4}$ Bashkir State University, Ufa, Russian Federation

On the risk of developing socially significant diseases and life expectancy among the younger generation, a significant contribution is made by behavioral stereotypes (smoking), neuroendocrine and genetic determinate psychological phenotypes. The study involved 116 healthy Yakut men, whose average age was $21.5 \pm 2.25$. The sample was divided into two groups: smokers $(\mathrm{n}=36)$ and non-smokers $(n=80)$. A statistically significant increase in the level of cortisol in smokers $(11.52 \mu \mathrm{g} / \mathrm{dl})$ was found compared with non-smokers $(9.28 \mu \mathrm{g} / \mathrm{dl})(\mathrm{p}=0.02)$. It was established that the average grade of extraversion and neuroticism is higher in smokers than in non-smokers (extraversion: 12.91 and 11.50, respectively, $\mathrm{p}=0.04$; neuroticism: 13.13 and 11.36 , respectively, $\mathrm{p}=0.04)$. The prevalence of choleric $(38.89 \%)$ and sanguine $(33.34 \%)$ temperament in the group of smokers was found in comparison with non-smokers $(22.50 \%$ and $20.00 \%$, respectively). Exposure to smoking from extraverts can be explained by psychological phenotypes of the basal personality traits characteristic for this type of temperament (impulsiveness, sociability, craving for 
new impressions). The dependence of the DHEA content in the blood serum of smokers on the extraversion rates $(r=$ $0.47, \mathrm{p}=0.02$ ) was shown: the more extraversion value was pronounced, the higher DHEA level was observed. Thus, the study showed that the status of smoking among Yakut men influenced on the level of steroid hormones and linked with psychological phenotypes. The study was supported by Ministry of Education and Science of Russia
№6.1766.2017, FASO BRK_0556-2017-0003, Grant of the Head of the Republic of Sakha (Yakutia) to young scientists, specialists and students for 2018 and RFBR (18-3400521_mol_a).

S.S. Nakhodkin: None. N.A. Barashkov: None. V.G. Pshennikova: None. A.V. Kazantseva: None. E.K. Khusnutdinova: None. N.N. Sazonov: None. S.A. Fedorova: None. 